\title{
Propriedades Térmicas e Magnéticas dos Borocarbetos
}

\section{Tese de Doutorado}

Tese apresentada ao Programa de Pós Graduação em Física do Departamento de Física da PUC-Rio como parte dos requisitos para obtenção do título de Doutor em Física.

Orientador: Prof. Carlos Mauricio Chaves

Rio de Janeiro, 5 de novembro de 2002 
Todos os direitos reservados. É proibida a reprodução total ou parcial do trabalho sem autorização da universidade, do autor e do orientador.

\section{Jaime Francisco Vento Flores}

Graduou-se em Física na UNMSM (Universidad Nacional Mayor de San Marcos, Lima - Perú) em 1988. Área de actuação é Física da Matéria Condensada.

Ficha Catalográfica

Vento Flores, Jaime Francisco

Propriedades térmicas e magnéticas dos borocarbetos / Jaime Francisco Vento Flores; orientador: Carlos Mauricio Chaves. - Rio de Janeiro : PUC, Departamento de Física, 2002.

[11], 54 f. : il. ; $30 \mathrm{~cm}$

Tese (doutorado) - Pontifícia Universidade Católica do Rio de Janeiro, Departamento de Física.

Inclui referências bibliográficas.

1. Física - Teses. 2. Borocarbetos. 3. Ondas de spin. 4. Calor específico. 5. Magnetização. I. Chaves, Carlos Mauricio. II. Pontifícia Universidade Católica do Rio de Janeiro. Departamento de Física. III. Título. 
Dedicatoria... 
A Ernestina,

minha querida mãe.

A Eulogio,

meu pai (In Memoriam)

A Leni Nélida minha esposa e a Lenny Victoria e Thiago Jonatan meus filhos. Pela sua paciência, pelo amor, carinho e força.

A Victoria (In Memoriam), Hugo Walter, Ana María, Washington José e Eulogio Titov. 



\section{Resumo}

Propomos um hamiltoniano de spin para descrever as propriedades térmicas e magnéticas de uma serie de borocarbetos. O efeito do campo elétrico cristalino é aproximado por uma campo magnético efetivo.

Aplicamos a teoria de onda de spin e encontramos a relação de dispersão e a densidade de estados dos magnons. Em presença de um campo magnético externo esta densidade apenas se desloca rigidamente em energia.

A partir destes resultados calculamos a contribuição dos magnons ao calor específico molar com e sem campo magnético externo. O efeito do campo externo, porém, é pequeno.

A seguir calculamos a magnetização de subrede a $T=0 K$, a qual sofre uma pequena redução devido às flutuações quânticas, em relação ao valor iônico obtido pela regra de Hund. Encontramos também a magnetização em função da temperatura, com e sem aplicação de campo magnético.

Com esse modelo conseguimos descrever bastante bem os dados experimentais de calor específico da serie.

\section{Palavras-chave}

Borocarbetos; ondas de spin; calor específico; magnetização. 


\section{Abstract}

We propose a spin hamiltonian to describe the magnetic and thermal properties of a series of borocarbides. The effect of the crystal electric field is simulated by an effective magnetic field.

By applying spin wave theory we have found the dispersion relation and the density of state for the magnos as well. In presence of an external magnetic field this density of state shifts rigidly in energy.

From these results we have calculated the magnon contribuition to the molar specific heat whith and whithout applied magnetic field. The effect of the field, however, is very small.

We also have found the sublattice magnetization at $T=0 K$ which is slightly reduced with respect to the ionic value given by the Hund's rule.

Then we have calculated the sublattice magnetization as a function of $\mathrm{T}$, both in presence and in absence of the magnetic field.

With this model we succeed in fitting fairly well the experimental data for the specific heat of the borocarbides series.

\section{Keywords}

Borocarbides; spin wave; specific heat; magnetization. 


\section{Agradecimentos}

Ao Prof. Carlos Mauricio Chaves minha gratidão por sua orientação, disponibilidade em todo o decurso do trabalho de tese e cuja convivência me ensinou e me amaducereu profissionalmente.

Ao Prof. Mohammed El Massalami pelas discussões úteis e os dados experimentais.

Ao Prof. Mucio Continentino e ao Prof. Pedro von Ranke pelos discussões úteis e sugestões ao presente trabalho.

A Profa. Vera Lúcia V. Baltar e ao Prof. Paulo Costa Ribeiro pela solidariedade e amizade nos momentos difíceis. Ao Pe. Paul Schweitzer, SJ, pela amizade e orientações e a Jorge Dau amigo e mentor.

Ao pessoal administrativo e funcionários do departamento de Física da PucRio pelo apoio e dedicação.

Aos colegas Lourival, Edson, Sarai, Anivaldo, Marcelo e aos compatriotas Richard, Walter, Dalber, Vicente e Rigoberto pela amizade.

Finalmente ao Brasil pela bolsa concedida para o programa de Doutorado, através do Conselho Nacional de Desenvolvimento Científico e Tecnológico (CNPq). 


\section{Sumário}

1 Introdução 11

2 Borocarbetos 14

$\begin{array}{ll}2.1 \text { Estrutura cristalina dos compostos } \mathrm{RNi}_{2} \mathrm{~B}_{2} \mathrm{C} & 14\end{array}$

$\begin{array}{ll}2.2 \text { Rede recíproca dos borocarbetos } & 15\end{array}$

2.3 Supercondutividade em borocarbetos não magnéticos 17

2.3.1 Propriedades eletrônicas do estado normal e do estado supercondutor 19

$\begin{array}{ll}\text { 2.3.2 Campo critico superior } & 20\end{array}$

2.4 Ordem magnética e supercondutividade nos borocarbetos 21

2.5 Ordem magnética e CEF 22

2.6 Estrutura magnética para $R=\mathrm{Gd}, \mathrm{Ho}, \mathrm{Er}, \mathrm{Dy}, \mathrm{Tb}$ e $\mathrm{Nd} 23$

2.6.1 $\mathrm{GdNi}_{2} \mathrm{~B}_{2} \mathrm{C}$

2.6.2 $\mathrm{HoNi}_{2} \mathrm{~B}_{2} \mathrm{C}$

2.6.3 $\mathrm{ErNi}_{2} \mathrm{~B}_{2} \mathrm{C}$

2.6.4 $\mathrm{DyNi}_{2} \mathrm{~B}_{2} \mathrm{C} \quad 25$

2.6.5 $\mathrm{TbNi}_{2} \mathrm{~B}_{2} \mathrm{C}$

2.6.6 $\mathrm{NdNi}_{2} \mathrm{~B}_{2} \mathrm{C} \quad 25$

2.7 Hipótese básica 26

3 Aproximação de ondas de spin $\quad 27$

3.1 hamiltoniano 27

3.2 Cálculo da relação de dispersão dos borocarbetos 27

$\begin{array}{lll}3.3 & \text { Densidade de estados } 33\end{array}$

4 Cálculo do calor específico em função da temperatura 36

4.1 Energia interna 36

4.2 Calor específico na fase AF 3D 36

4.2.1 Limite de altas temperaturas $(T \gg \Delta) \quad 38$

4.2.2 Limite de baixas temperaturas $(T \ll \Delta) \quad 38$

4.3 Caso quase-bidimensional 39

$\begin{array}{lll}4.4 & \text { Aplicações aos borocarbetos } & 39\end{array}$

4.4.1 $\mathrm{GdNi}_{2} \mathrm{~B}_{2} \mathrm{C} \quad 39$

$\begin{array}{ll}\text { 4.4.2 } \mathrm{HoNi}_{2} \mathrm{~B}_{2} \mathrm{C} & 40\end{array}$ 
4.4.3 $\mathrm{ErNi}_{2} \mathrm{~B}_{2} \mathrm{C} \quad 40$

4.4.4 $\mathrm{DyNi}_{2} \mathrm{~B}_{2} \mathrm{C}$

4.4.5 $\mathrm{TbNi}_{2} \mathrm{~B}_{2} \mathrm{C}$

4.4.6 $\mathrm{NdNi}_{2} \mathrm{~B}_{2} \mathrm{C}$

5 Calor específico em função da temperatura e do campo magnético externo 46

$\begin{array}{lll}5.1 & \text { Densidade de estados } & 46\end{array}$

5.2 Energia Interna 47

$\begin{array}{lll}5.3 \text { Calor específico } & 48\end{array}$

6 Magnetização 52

6.1 Magnetização de subrede 52

6.2 Magnetização da subrede a $T=0 K$

6.3 Magnetização da subrede a $T \neq 0 K$

6.3.1 Limite de baixas temperaturas $T \ll \Delta \quad 56$

6.4 Magnetização total 58

7 Conclusão $\quad 60$

$\begin{array}{ll}\text { Referências } & 61\end{array}$ 


\section{Lista de Figuras}

2.1 A estrutura cristalina dos borocarbetos $R N i_{2} B_{2} C$ é uma rede de Bravais tetragonal de corpo centrado.

2.2 Parâmetros da rede da serie $R N i_{2} B_{2} C$ em função do raio iônico da TR

2.3 Estrutura cristalina dos borocarbetos $R_{n} N i_{2} B_{2} C_{n}$.

2.4 Primeira Zona de Brillouin de uma rede tetragonal de corpo centrado e pontos de alta simetria.

2.5 Densidade de estados parcial e total do $L u N i_{2} B_{2} C$ e $Y N i_{2} B_{2} C$, na aproximação da densidade local (LDA) . 19

2.6 $T_{C}$ e $T_{N}$ em função do fator de de Gennes dos borocarbetos.

2.7 Estruturas magnética do (a) Pr, Dy e Ho; (b) Er; (c) Tb; (d) Tm; (e) $\mathrm{Er} \mathrm{Ni} i_{2} \mathrm{~B}_{2} \mathrm{C}$ mostrando sobre varias células unitarias .

2.8 Estrutura magnética do: (a) $N d N i_{2} B_{2} C$, (b) e (c) $G d N i_{2} B_{2} C \quad$. 24

3.1 Ilustração da divisão em subredes no hamiltoniano (3.1).

3.2 Espectro de energia das ondas de spin na fase AF com campo magnético aplicado.

3.3 Relação de dispersão completa (3.29) e aproximada (3.36) das ondas de spin.

3.4 Densidade de estados em função da energia.

4.1 Calor específico do $G d N i_{2} B_{2} C$. A linha sólida correspode a resultados teóricos para $c_{1} \sqrt{c_{2}}=173.9 K^{3}$ e os pontos representam resultados experimentais .

4.2 Calor específico do $\mathrm{HoNi}_{2} \mathrm{~B}_{2} \mathrm{C}$. A linha sólida correspode a resultados teóricos para $c_{1} \sqrt{c_{2}}=87.8 K^{3}$ e os pontos representam resultados experimentais .

4.3 Calor específico do $\operatorname{ErNi} i_{2} B_{2} C$; a linha sólida correspode a resultados teóricos para $c_{1} \sqrt{c_{2}}=38.9 K^{3}$ e os pontos representam resultados experimentais .

4.4 Calor específico do $D y N i_{2} B_{2} C$; a linha sólida correspode a resultados teóricos para $J_{1}=3.8 \mathrm{~K}$ e os pontos representam resultados experimentais

4.5 Calor específico do $T b N i_{2} B_{2} C$. A linha sólida correspode a resultados teóricos para $c_{1} \sqrt{c_{2}}=1691,6 K^{3}$ e os pontos representam resultados 
experimentais. A linha pontilhada correspode a resultados teóricos para o $N d N i_{2} B_{2} C\left(c_{1} \sqrt{c_{2}}=50.5 K^{3}\right)$ e os pontos representam resultados experimentais .

$4.6 J_{1}$ vs. $J_{2}$ para o $\mathrm{HoNi}_{2} \mathrm{~B}_{2} \mathrm{C}$.

4.7 Calor específico do $\mathrm{HoNi}_{2} \mathrm{~B}_{2} \mathrm{C}$. A linha sólida representa resultados experimentais e a linha pontilhada e os pontos representam resultados teóricos usando a relação analítica e integrando na ZB respectivamente para $c_{1} \sqrt{c_{2}}=87,8 K^{3}$.

5.1 Magnetização $\mathrm{M}$ vs campo magnético aplicado $\mathrm{H}$ para o $\mathrm{HoNi} i_{2} \mathrm{~B}_{2} \mathrm{C}$ em $T=2 K$ para $\mathrm{H} \| c$ e $\mathrm{H} \perp c$. Para $\mathrm{H} \perp c$ o campo magnético faz um angulo $\theta$ com relação ao eixo cristalográfico [110] .

5.2 Densidade de estados dos magnons com um campo magnético aplicado. 48

5.3 Calor específico do $\mathrm{HoN}_{2} \mathrm{~B}_{2} \mathrm{C}$ em função do campo magnético aplicado para $T=2 K$, para $c_{1} \sqrt{c_{2}}=87.8 K^{3}$.

5.4 Calor específico do $\mathrm{HoN}_{2} \mathrm{~B}_{2} \mathrm{C}$ em função do campo magnético aplicado para $T=2 K$, para $c_{1} \sqrt{c_{2}}=87.8 K^{3}$.

6.1 Variação de $\Delta S_{A}^{z}(T=0 K)$ vs. $J_{2}$ para o $H o N i_{2} B_{2} C$.

6.2 Magnetização da subrede $S_{A}^{z}$ vs. T para o $\mathrm{HoNi}_{2} \mathrm{~B}_{2} \mathrm{C}$ na ausência de campo magnético externo.

6.3 Magnetização da subrede $S_{A}^{z}$ vs. T para um campo magnético aplicado de $H_{\text {ext }}=0.0 \mathrm{~T}, 1.0 \mathrm{~T}$ e $4.0 \mathrm{~T}$ para o $\mathrm{HoNi}_{2} \mathrm{~B}_{2} \mathrm{C}$.

6.4 Magnetização da subrede $S_{A}^{z}$ vs. campo magnético aplicado a $T=2 \mathrm{~K}$ para o $\mathrm{HoNi}_{2} \mathrm{~B}_{2} \mathrm{C}$.

6.5 Magnetização total $\left\langle S^{z}>\right.$ vs. $H_{\text {ext }}$ (em Tesla) a $\mathrm{T}=2 \mathrm{~K}$ para o $\mathrm{HoNi}_{2} \mathrm{~B}_{2} \mathrm{C}$. 


\section{1 \\ Introdução}

O descobrimento dos borocarbetos ${ }^{1-4}$ foi anunciado faz aproximadamente sete anos e neste curto espaço de tempo ocorreu um progresso espetacular no entendimento destes fascinantes materiais. Os borocarbetos são uma familia de compostos com fórmula $R \mathrm{Ni}_{2} B_{2} C$ onde $\mathrm{R}$ denota uma terra rara (TR). Uma das razões para este rápido progresso são os processos de síntese desta classe de materiais, os quais tem permitido fazer amostras policristalinas e monocristalinas de alta qualidade ${ }^{5,6}$. A disponibilidade de amostras de alta qualidade permite a obtenção de resultados experimentais mais precisos, o que é crítico para o desenvolvimento de teorias que permitam um entendimento de qualquer classe de materiais; para os borocarbetos isto permitiu um rápido progresso. Uma das intrigantes propriedades destes materiais é que eles apresentam naturalmente ordem supercondutora e ordem magnética de longo alcance na mesma faixa de temperatura as quais competem e coexistem a nível microscópico.

Os borocarbetos dividem algumas caraterísticas gerais com outra familia de materiais magnéticos e supercondutores: os boratos de Ródio e as fases de Chevrel (chamados de antigos supercondutores magnéticos). Apesar da diferença na estrutura e nas propriedades químicas, os mesmos elementos parecem dominar o comportamento destes compostos. Consequentemente são agrupados na mesma classe de supercondutores magnéticos.

A principal característica comum entre os supercondutores magnéticos é a presença de duas classes de elétrons, com diferentes graus de localização: os elétrons $4 f$ das $\mathrm{TR}$, com fortes propriedades magnéticas anisotrópicas e os elétrons dos orbitais $d$ dos metais de transição que formam uma banda e que são responsáveis pelo comportamento metálico. Esta clara separação entre os graus de liberdade magnético e metálico e a fraca interação entre os dois, distinguem os borocarbetos e os antigos supercondutores magnéticos dos outros materiais, os supercondutores de óxido de cobre de alto $T_{C}$ e os fermions pesados, onde as propriedades supercondutoras e magnéticas são devido somente a uma classe de elétrons, tipicamente os elétrons de condução. Por conseguinte, os cupratos e fermions pesados não pertecem à esta classe de compostos e devem ser tratados com um diferente enfoque teórico.

Com relação às propriedades magnéticas destes compostos, eles 
apresentam uma variedade de estruturas magnéticas, as quais serão discutidas no próximo capítulo:

- Comensurável antiferromagnético (AF).

- Onda de densidade de spin (SDW) incomensurável modulada no eixo $a$, com vetor de onda $\tau$.

- Helicoidal ao longo do eixo $c$.

- Ferromagnetismo fraco (WFM).

- Spin-flop, etc.

(Para a definição dos eixos $a, b$ e $c$ ver figura 2.1) De particular interesse é o caso do $\mathrm{HoNi}_{2} \mathrm{~B}_{2} \mathrm{C}$, onde os três primeiros tipos de ordenamento magnético foram observados ${ }^{7}$. Se observou neste composto que a estrutura magnética incomensurável está relacionada ao comportamento reentrante do estado supercondutor e a coexistência do estado comensurável AF com a supercondutividade ${ }^{8}$.

A estrutura magnética dos borocarbetos depende do elemento $\mathrm{R}$ e das distâncias entre as camadas R-C que estão separadas pelas camadas de Ni-B. Nos compostos $R N i_{2} B_{2} C$ que se ordenam magneticamente (na sua maioria antiferromagneticamente) as temperaturas de transição variam ${ }^{9,10}$ desde $T_{N}=19.4 \mathrm{~K}$ para o $G d N i_{2} B_{2} C$ até $T_{N}=1.5 \mathrm{~K}$ para o $T m N i_{2} B_{2} C$. As variações de $T_{N}$ e $T_{C}$ seguem razoavelmente o fator de de Gennes, desde o Lu até o Gd e desde o Lu até o Tb, respectivamente, com exceção do Yb (ver figura 2.6). Isto implica que o acoplamento entre os momentos magnéticos é feito principalmente via as interações indiretas dos íons através dos elétrons de condução (interação RKKY), sendo a interação dipolar magnética menos importante $\mathrm{e}^{11}$.

Contudo, os estudos de vários compostos pseudoquaternarios $\left(R, R^{\prime}\right) \mathrm{Ni}_{2} \mathrm{~B}_{2} \mathrm{C}$ mostrou que o escalamento pelo fator de de Gennes não é universal para os borocarbetos, dependendo da estrutura eletrônica, do campo cristalino, da diferença nos raios iônicos de $R$ e R' ou de efeitos de impurezas não magnéticas em compostos com $T_{C}<T_{N}$.

O fato que $T_{C}, T_{N}$ assim como o comprimento de coerência do supercondutor $\left(\xi_{0}\right)$ e o livre caminho médio eletrônico $(l)$ podem ser sintonizados por uma escolha apropriada do elemento $\mathrm{R}$ e a substituição do metal de transição, permite um estudo sistemático seja dos supercondutores magnéticos $\left(T_{C}>\right.$ $\left.T_{N}\right)$ ou dos magnetos supercondutores $\left(T_{C}<T_{N}\right)$.

O vetor de modulação $\tau$, na ordem magnética incomensurável em vários compostos $R N i_{2} B_{2} C$ com $\mathrm{R}$ magnéticos e não magnéticos, está relacionado 
com o vetor de encaixamento perfeito (nesting) na superficie de Fermi. Também, admite-se que a supercondutividade seja mediada pelos fônons e uma manifestação direta deste acoplamento é observado no espectro dos fônons e no vetor de onda de encaixamento perfeito (ao longo do eixo $a$ ).

Do ponto de vista experimental, a estrutura magnética incomensurável ao longo do eixo- $a$, no $\mathrm{HoN}_{2} \mathrm{~B}_{2} \mathrm{C}$, ainda não foi totalmente esclarecida.

Os resultados da aplicação de um campo magnético a estas estruturas mostram que alguns compostos $R \mathrm{Ni}_{2} \mathrm{~B}_{2} \mathrm{C}$ sofrem transições metamagnéticas. Tem-se estudado também o efeito do campo magnético nas propriedades térmicas desses compostos.

O principal objetivo do presente trabalho é proporcionar um modelo que calcule a contribuição dos magnons ao calor específico e à magnetização nos borocarbetos. Conseguimos ajustar os resultados experimentais obtidos pelo grupo de baixas temperaturas do Instituto de Física da UFRJ e são esses resultados ${ }^{12}$ para a contribuição magnética ao calor específico que são apresentados no capítulo 4. Para todos os compostos o calor específico foi analizado como a soma de um termo da rede $c_{L}=\beta T^{3}$, um termo eletrônico $c_{e l}=\gamma T$ (ou um equivalente no caso supercondutor), uma contribuição magnética e finalmente um termo nuclear. As contribuições não magnéticas foram então ajustadas tomando como referência o composto isomorfo $Y \mathrm{Ni}_{2} \mathrm{~B}_{2} \mathrm{C}$ o que permitiu a extração da parte magnética da série $^{12}$. É proposto um hamiltoniano de spin fenomenológico baseado no qual calculamos as propriedades térmicas e magnéticas de uma serie de compostos $R \mathrm{Ni}_{2} \mathrm{~B}_{2} \mathrm{C}$ ( $R=\mathrm{Ho}, \mathrm{Er}, \mathrm{Pr}, \mathrm{Gd}, \mathrm{Dy}$ ). O caso do $\mathrm{HoN} i_{2} \mathrm{~B}_{2} \mathrm{C}$ é discutido com mais detalhe.

A continuação damos um breve resumo dos temas que serão abordados: no capítulo 2 damos uma descrição dos borocarbetos. No capítulo 3 apresentamos nosso modelo, o hamiltoniano de spin, a teoria de ondas de spin e calculamos a relação de dispersão dos magnons. No capítulo 4 são apresentados os cálculos do calor específico sem campo magnético externo $c_{m}\left(T, H_{\text {ext }}=0\right)$ e no capítulo 5 com campo magnético externo $c_{m}\left(T, H_{\text {ext }}\right)$ os quais são comparados com os resultados experimentais. No capítulo 6 apresentamos os cálculos da magnetização da sub-rede a $T=0 K$ e assim como em função da temperatura e do campo magnético externo e finalmente no capítulo 7 apresentamos as conclusões. 


\section{2 \\ Borocarbetos}

\subsection{Estrutura cristalina dos compostos $\mathrm{RNi}_{2} \mathrm{~B}_{2} \mathrm{C}$}

Os borocarbetos são uma classe de compostos com fórmula $R \mathrm{Ni}_{2} \mathrm{~B}_{2} \mathrm{C}$, onde $\mathrm{R}$ é um ion trivalente $\left(R^{3+}\right)$. Compostos estáveis foram sintetizados com $\mathrm{R}=\mathrm{Sc}$, Y, La ou uma terra rara (TR). Todos estes compostos têm a mesma rede tetragonal de corpo centrado (bct) com simetria de grupo espacial $I 4 / \mathrm{mmm}^{3,13}$ mostrado na figura 2.1.

A estrutura consiste em planos R-C que se alternam com camadas de $N i_{2} B_{2}$ tetraédricos, empilhados ao longo do eixo $c$. Quatro átomos de B estão localizados nos vértices de um tetraedro ligeiramente distorcido centrado no sitio do $\mathrm{Ni}$. Os enlaces da rede também incluem conexões B-C e apesar da aparente estrutura em camadas, o comportamento ${ }^{14,15}$ é 3D . Os ions $R^{3+}$ estão localizados no centro de uma grande cavidade e tem um enlace fraco com o resto da estrutura; isto faz com que a dopagem sobre estes sitios seja particularmente fácil.

Os parâmetros de rede $a$ e $b$ foram determinados experimentalmente para quase todos componentes da familia ${ }^{5,13}$ e são aproximadamente $a=3.5 \AA$ e $c=10.5 \AA$, com $a=b$ pela simetria tetragonal. As estruturas dos compostos $R \mathrm{Ni}_{2} \mathrm{~B}_{2} C$ são altamente anisotrópicas com uma razão de $c / a$ de aproximadamente 3. Da figura 2.2, se ve que o parâmetro a aumenta e o parâmetro $c$ decrese com o raio iônico das TR, resultando em um aumento do volume. Esta variação regular dos parâmetros de rede não inclui o $\mathrm{CeN} i_{2} \mathrm{~B}_{2} \mathrm{C}$ devido ao comportamento de valência intermediária dos elétrons $4 f$ do Cerio $^{16,17}$. As particularidades na estrutura destes compostos se refletem nas suas propriedades magnéticas e supercondutoras.

Uma característica importante dos borocarbetos é que nestes compostos é possível adicionar planos de R-C entre as camadas adjacentes de $N i_{2} B_{2}$ o qual permite a formação de uma nova classe de $\operatorname{compostos}^{3,18}$ de fórmula $R_{n} N i_{2} B_{2} C_{n}(\mathrm{n}=1,2, \ldots)$, não observada nos compostos ternários (os antigos supercondutores magnéticos: as fases de Chevrel e os boratos de Rhodio). Os compostos mais conhecidos são para $n=1$ e $n=2$ (ver figuras 2.1 e 2.3), porém também foi sintetizado ${ }^{20,21}$ a serie até $n=4$. Nesta tese estudamos compostos para $n=1$. 


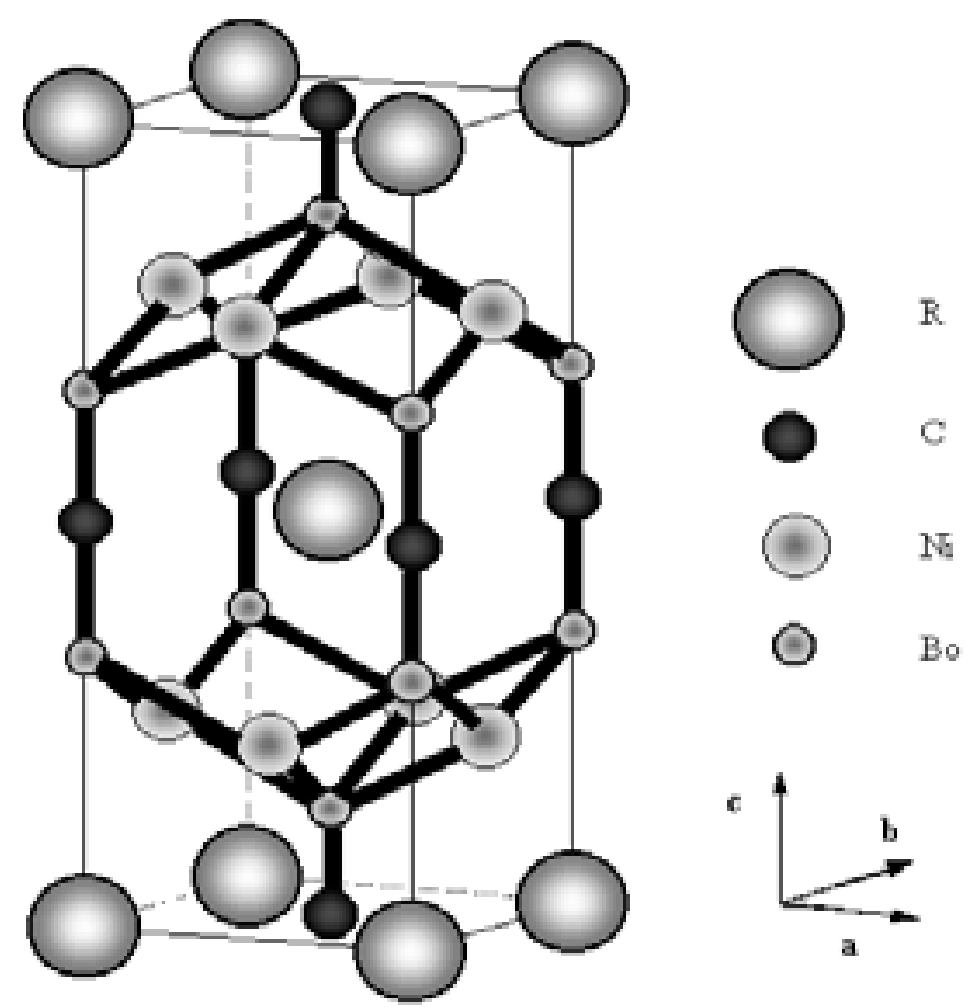

Figura 2.1: A estrutura cristalina dos borocarbetos $R N i_{2} B_{2} C$ é uma rede de Bravais tetragonal de corpo centrado.

\subsection{Rede recíproca dos borocarbetos}

A rede recíproca dos borocarbetos tem simetria tetragonal de face centrada e a zona de Brillouin correspondente é mostrada na figura 2.4. Os vetores base da rede recíproca são:

$$
\begin{aligned}
a^{*} & =2 \pi\left(\frac{1}{a} \hat{x}+\frac{1}{a} \hat{y}\right) \\
b^{*} & =2 \pi\left(\frac{1}{a} \hat{y}+\frac{1}{c} \hat{z}\right) \\
c^{*} & =2 \pi\left(\frac{1}{a} \hat{x}+\frac{1}{c} \hat{z}\right)
\end{aligned}
$$

Então qualquer vetor $\vec{k}$ da rede recíproca tem a forma:

$$
\vec{k}=n a^{*}+m b^{*}+l c^{*}
$$

onde $n, m$ e $l$ são números inteiros.

As coordenadas dos principais pontos de simetria da zona de Brillouin (ver fig. 2.4) são:

$$
\begin{aligned}
& \Gamma=(0,0,0) \\
& Z=\frac{2 \pi}{c}(0,0,1)
\end{aligned}
$$



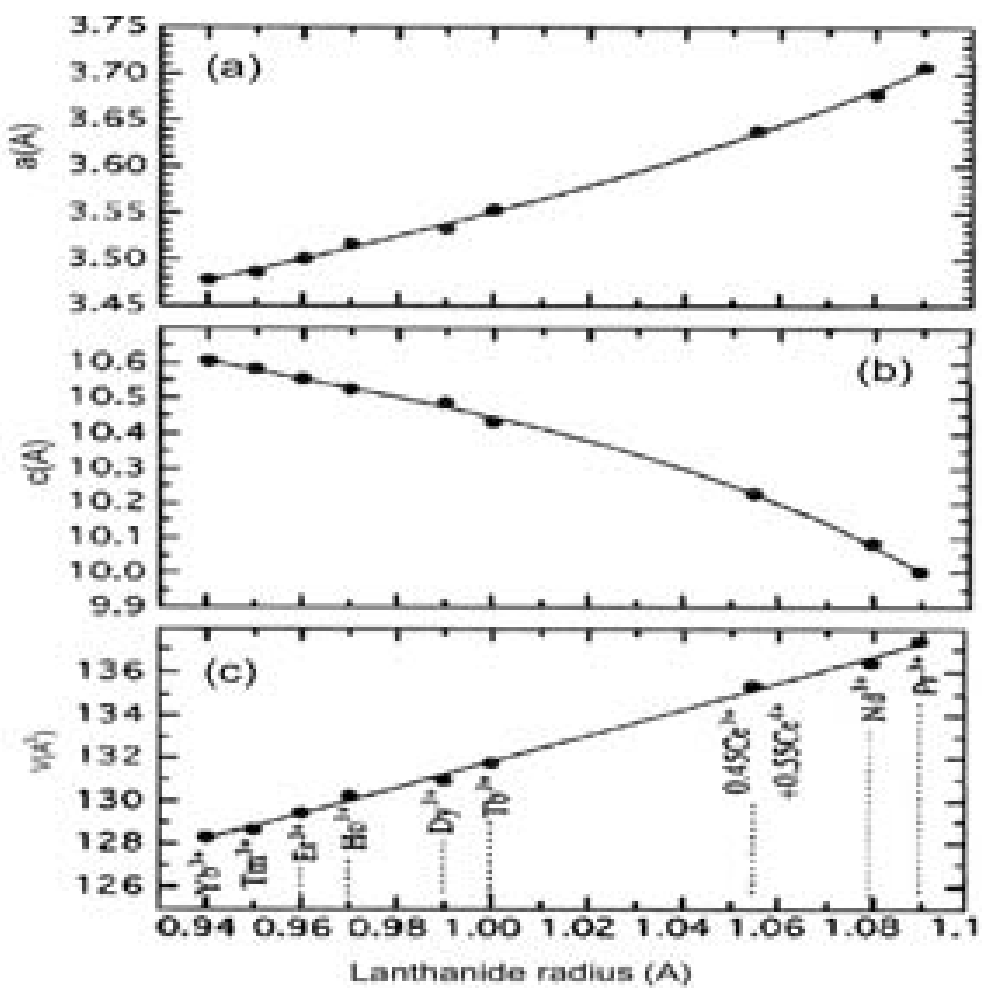

Figura 2.2: Parâmetros da rede da serie $R N i_{2} B_{2} C$ em função do raio iônico da $\mathrm{TR}^{5}$.
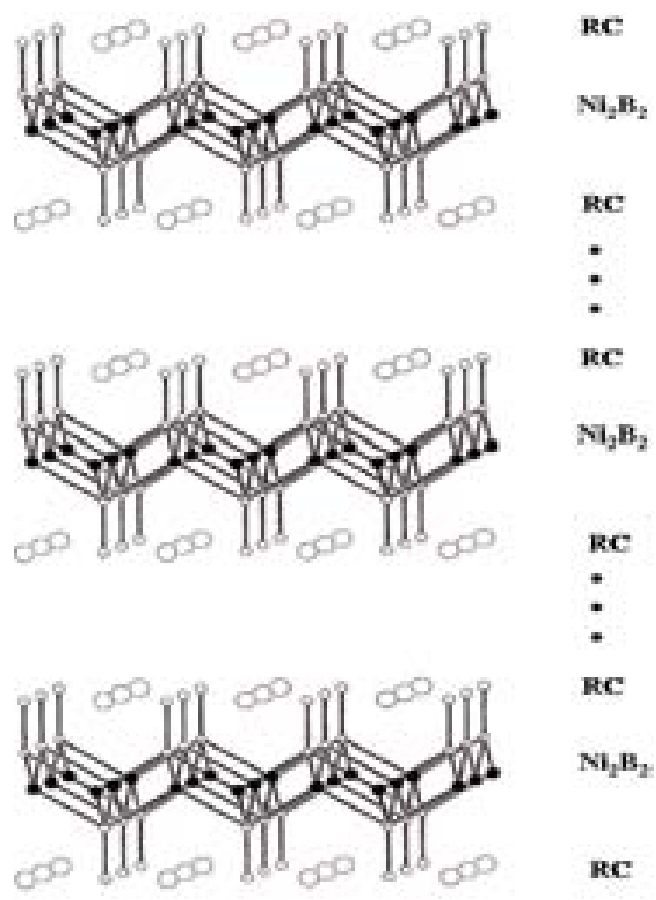

Figura 2.3: Estrutura cristalina dos borocarbetos $R_{n} N i_{2} B_{2} C_{n}{ }^{18}$. 


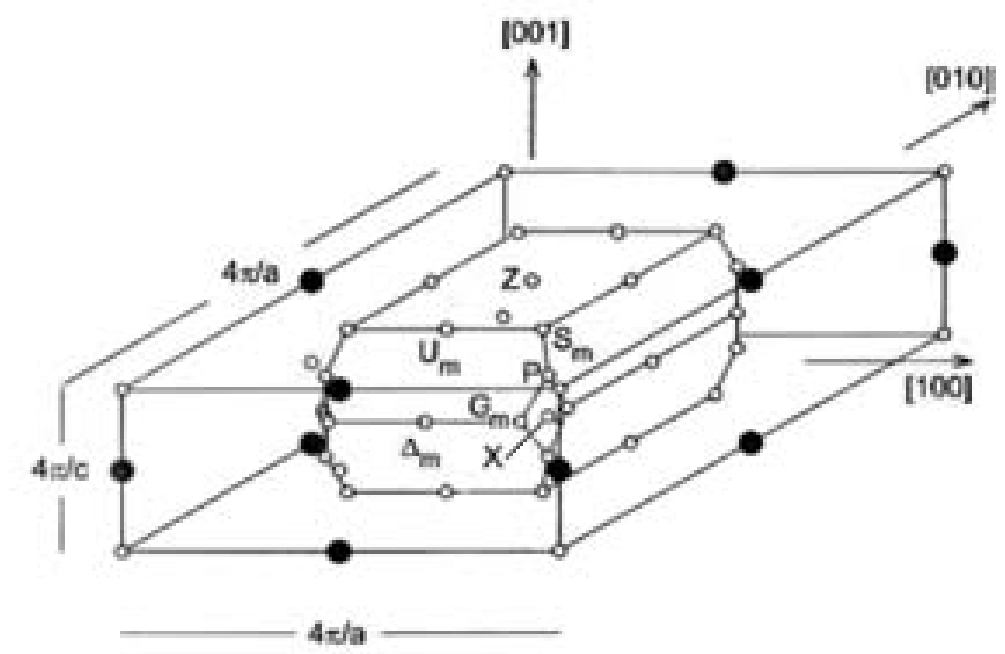

Figura 2.4: Primeira Zona de Brillouin de uma rede tetragonal de corpo centrado e pontos de alta simetria ${ }^{19}$.

$$
\begin{aligned}
X & =\frac{2 \pi}{c}\left(\frac{c}{2 a}, \frac{c}{2 a}, 0\right) \\
P & =\frac{2 \pi}{c}\left(\frac{c}{2 a}, \frac{c}{2 a}, \frac{1}{2}\right) \\
\Delta_{m} & =\frac{2 \pi}{c}\left[\frac{1}{2}\left(\frac{c}{a}+\frac{a}{c}\right), 0,0\right] \\
U_{m} & =\frac{2 \pi}{c}\left[\frac{1}{2}\left(\frac{c}{a}-\frac{a}{c}\right), 0,1\right] \\
V & =\frac{2 \pi}{c}\left(\frac{c}{2 a}, 0, \frac{1}{2}\right) \\
S_{m} & =\frac{2 \pi}{c}\left[\frac{1}{2}\left(\frac{c}{a}-\frac{a}{c}\right), \frac{1}{2}\left(\frac{c}{a}-\frac{a}{c}\right), 1\right] \\
G_{m} & =\frac{2 \pi}{c}\left[\frac{1}{2}\left(\frac{c}{a}+\frac{a}{c}\right), \frac{1}{2}\left(\frac{c}{a}-\frac{a}{c}\right), 0\right]
\end{aligned}
$$

\subsection{Supercondutividade em borocarbetos não magnéticos}

Os borocarbetos são divididos em duas classes de compostos, com relação a suas propriedades magnéticas. Nesta seção veremos brevemente os supercondutores $R \mathrm{Ni}_{2} \mathrm{~B}_{2} \mathrm{C}$ com elementos $\mathrm{R}$, cujos íons $R^{3+}$ têm momento angular total zero no seu estado fundamental obtido segundo a regra de Hund, os chamados supercondutores borocarbetos não magnéticos. Corresponde a esta serie os elementos $\mathrm{R}=\mathrm{Sc}, \mathrm{Y}, \mathrm{Lu}$, Th. Na tabela 2.1 são apresentadas as temperaturas das transições supercondutoras dos borocarbetos supercondutores magnéticos e não magnéticos e as temperaturas do ordenamento magnético dos primeiros. 


\begin{tabular}{||c|c|c||}
\hline \hline Composto & $T_{C}(K)$ & $T_{N}(K)$ \\
\hline$C e N i_{2} B_{2} \mathrm{C}$ & 0.1 & - \\
\hline$D y N i_{2} B_{2} \mathrm{C}$ & 6.2 & 11 \\
\hline$H o N i_{2} B_{2} \mathrm{C}$ & 8 & 5 \\
\hline$E r N i_{2} B_{2} \mathrm{C}$ & 10.5 & 6.8 \\
\hline$T m N i_{2} B_{2} \mathrm{C}$ & 11 & 1.5 \\
\hline$L u N i_{2} B_{2} \mathrm{C}$ & 16.5 & - \\
\hline$S c N i_{2} B_{2} \mathrm{C}$ & 15.5 & - \\
\hline$T h N i_{2} B_{2} \mathrm{C}$ & 8 & - \\
\hline$Y N i_{2} B_{2} \mathrm{C}$ & 15.5 & - \\
\hline \hline
\end{tabular}

Tabela 2.1: Supercondutores borocarbetos magnéticos e não magnéticos: temperatura de transição supercondutora $T_{C}$ e temperatura de ordem magnética $T_{N}{ }^{7}$.

\begin{tabular}{||c|c|c|c|c|c|c||}
\hline \hline$R^{3+}$ & $\mathrm{n}$ & $\mathrm{S}$ & $\mathrm{L}$ & $\mathrm{J}$ & $g_{J}$ & $d \mathrm{G}$ \\
\hline $\mathrm{Ce}$ & 1 & $1 / 2$ & 3 & $5 / 2$ & $6 / 7$ & 0.18 \\
\hline $\mathrm{Pr}$ & 2 & 1 & 5 & 4 & $4 / 5$ & 0.80 \\
\hline $\mathrm{Nd}$ & 3 & $3 / 2$ & 6 & $9 / 2$ & $8 / 11$ & 1.8 \\
\hline $\mathrm{Sm}$ & 5 & $5 / 2$ & 5 & $5 / 2$ & $2 / 7$ & 4.56 \\
\hline $\mathrm{Gd}$ & 7 & $7 / 2$ & 0 & $7 / 2$ & 2 & 15.8 \\
\hline $\mathrm{Tb}$ & 8 & 3 & 3 & 6 & $3 / 2$ & 10.5 \\
\hline $\mathrm{Dy}$ & 9 & $5 / 2$ & 5 & $15 / 2$ & $4 / 3$ & 7.1 \\
\hline $\mathrm{Ho}$ & 10 & 2 & 6 & 8 & $5 / 4$ & 4.5 \\
\hline $\mathrm{Er}$ & 11 & $3 / 2$ & 6 & $15 / 2$ & $6 / 5$ & 2.6 \\
\hline $\mathrm{Tm}$ & 12 & 1 & 5 & 6 & $7 / 6$ & 1.2 \\
\hline $\mathrm{Yb}$ & 13 & $1 / 2$ & 3 & $7 / 2$ & $8 / 7$ & 0.32 \\
\hline $\mathrm{Lu}$ & 14 & 0 & 0 & 0 & - & 0 \\
\hline \hline
\end{tabular}

Tabela 2.2: Propriedades dos ions livres $R^{3+}$. 

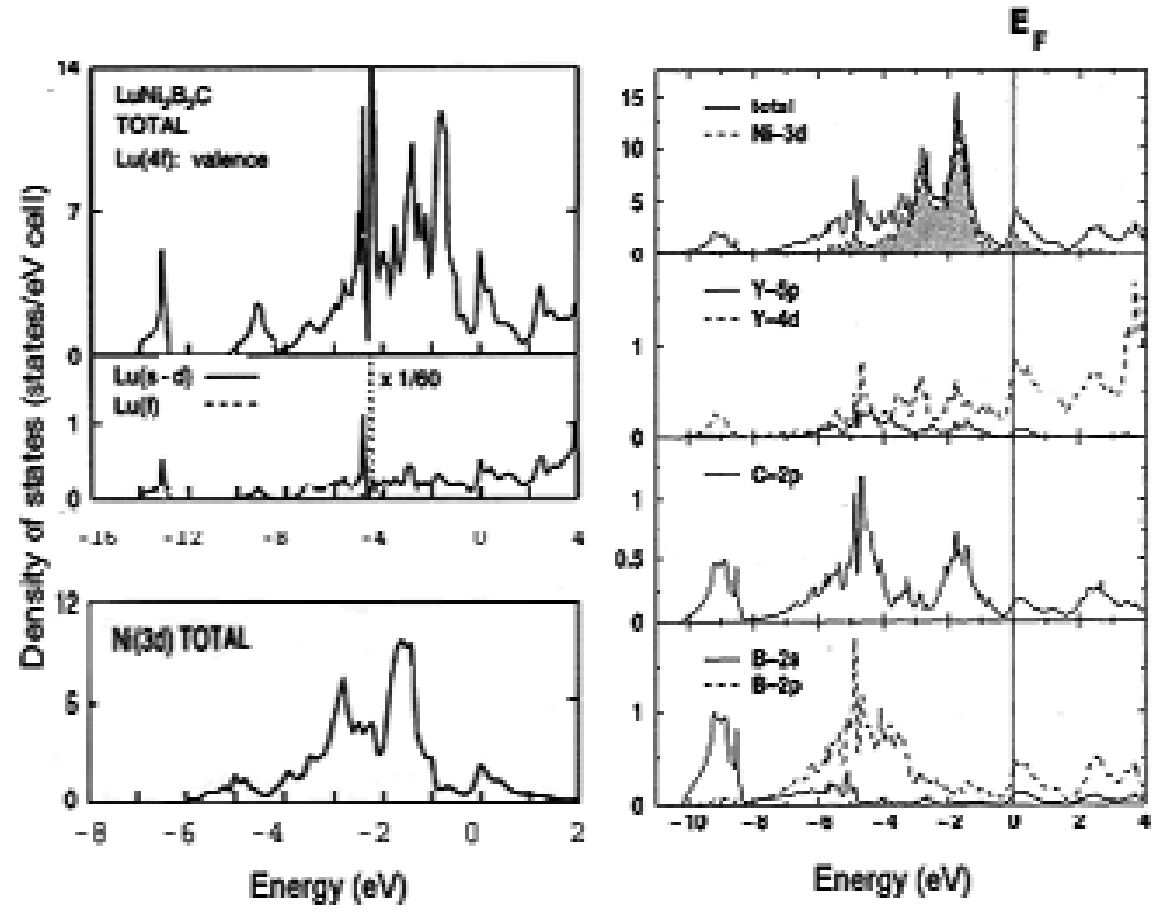

Figura 2.5: Densidade de estados parcial e total do $L u N i_{2} B_{2} C$ e $Y N i_{2} B_{2} C$, na aproximação da densidade local (LDA) ${ }^{14,23}$.

A característica geral da supercondutividade nos borocarbetos não magnéticos, tais como o pico no calor específico $(\Delta C)_{T_{C}}$, a curva do campo critico superior $H_{C_{2}}$ e a razão BCS $\Delta(0) / k_{B} T_{C}$, sugere que o mecanismo de apareamento são os fônons com um acoplamento de intermediário a forte ${ }^{22}$ apesar de que os cálculos dos parâmetros de interação varie consideravelmente $\left(\lambda_{p h} \approx 0.5-2\right)$. Contudo, foi mostrado experimentalmente que estes sistemas apresentam algumas anomalias, como veremos a seguir.

\subsubsection{Propriedades eletrônicas do estado normal e do estado supercondutor}

Os cálculos de estrutura eletrônica, mostram que os borocarbetos ${ }^{14,23}$, são metais 3D, apesar de apresentar uma estrutura em camadas, com todos os átomos contribuindo ao caráter metálico, ainda que a contribuição principal à densidade de estados no nível de Fermi, $N\left(E_{F}\right)$, surge dos elétrons $3 \mathrm{~d}$ do Ni. Há porém, uma considerável mistura de elétrons ${ }^{15} 5 d$ (no Y e no Lu), bem como $2 p$ (do B e do C). A figura 2.5 mostra a densidade de estados total e parcial dos compostos $(L u, Y) N i_{2} B_{2} C$, cujas propriedades eletrônicas são as mais tipicas da serie $R N i_{2} B_{2} C$.

Na aproximação de acoplamento fraco da teoria ${ }^{24}$ de Barden - Cooper - 
Schrieffer (BCS) da supercondutividade a temperatura de transição aumenta com $N\left(E_{F}\right)$, de acordo com a relação:

$$
T_{C}=1.13 \Theta_{D} e^{-1 / N\left(E_{F}\right) V}
$$

onde $\Theta_{D}$ é a temperatura de Debye caracterizando o espectro de fônons do material, V caracteriza a interação elétron - fônon e $N\left(E_{F}\right) V \ll 1$ é uma condição para dedução da equação (2.4). Embora a supercondutividade nos borocarbetos provavelmente seja mediada por fônons, não há uma conexão simples entre $T_{C}$ e $N\left(E_{F}\right)$ para toda a série. Por exemplo, no caso do $L a N i_{2} B_{2} C$ o valor de $N\left(E_{F}\right)$ é aproximadamente ${ }^{14}$ a metade do que para o $Y N i_{2} B_{2} C$ e foi argumentado, de acordo com a equação 2.4, que esta é a razão pela qual o $\mathrm{LaNi}_{2} \mathrm{~B}_{2} \mathrm{C}$ não é supercondutor. Contudo, foi assinalado que o supercondutor ${ }^{25} \mathrm{LaPt}_{2} \mathrm{~B}_{2} \mathrm{C}$ com $T_{C} \approx 11 \mathrm{~K}$, tem um similar ou ainda menor valor de $N\left(E_{F}\right)$ comparado com $L_{a N i} B_{2} C$. Então deve existir uma considerável variação dos parâmetros $\Theta_{D}$ ou $\mathrm{V}$ através da serie dos borocarbetos quaternários ${ }^{26}$ que afetam $T_{C}$ de acordo com a equação (2.4) ou estes materiais não são supercondutores tipo BCS. Um importante efeito isotópico do Boro foi observado no $\mathrm{YNi}_{2} \mathrm{~B}_{2} \mathrm{C}$ assim como no $\mathrm{LuNi} i_{2} \mathrm{~B}_{2} C$, que sustenta a classificação destes materiais como supercondutores mediados pelo acoplamento elétron - fônons ${ }^{27}$.

\subsubsection{Campo critico superior}

O campo crítico superior $H_{C_{2}}$ do $L u N i_{2} B_{2} C$ e $Y N i_{2} B_{2} C$ mostra

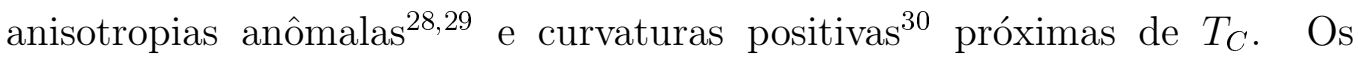
borocarbetos supercondutores estão em geral no limite puro, i.e., o livre caminho medio $\left(l_{0}\right)$ excede o comprimento de coerência $\left(\xi_{0}\right)\left(l_{0} \gg \xi_{0}\right)$. Em consequência $H_{C_{2}}$ é determinado principalmente pela estrutura eletrônica e os valores altos de $H_{C_{2}}$ requerem amostras puras, enquanto que para supercondutores impuros $\left(l_{0} \ll \xi_{0}\right) H_{C_{2}}$ aumenta com a concentração de impurezas. A dependencia não convencional de $H_{C_{2}}(T)$, mostrando uma curvatura positiva perto de $T_{C}$ pode ser explicada através da teoria de Eliashberg, usando um modelo de duas bandas ${ }^{30}$ assumindo dois grupos de eléctrons com velocidades de Fermi $\left(v_{F}\right)$ diferentes.

Estes fatos foram atribuidos a termos não locais na energia livre de Landau-Ginzburg ou alternativamente à anisotropia na estrutura de bandas. Vimos na seção anterior que os borocarbetos tem uma estrutura eletrônica aproximadamente 3D, porém a superfície de Fermi é altamente anisotrópica produzindo grandes mudanças na velocidade de Fermi ${ }^{23,31}\left(v_{F}\right)$ : estas predições foram confirmadas teórica e experimentalmente em compostos pseudoquaternarios de $\mathrm{YNi}_{2} \mathrm{~B}_{2} \mathrm{C}$ e $L u N i_{2} B_{2} C$ (isto é, considerando o efeito 


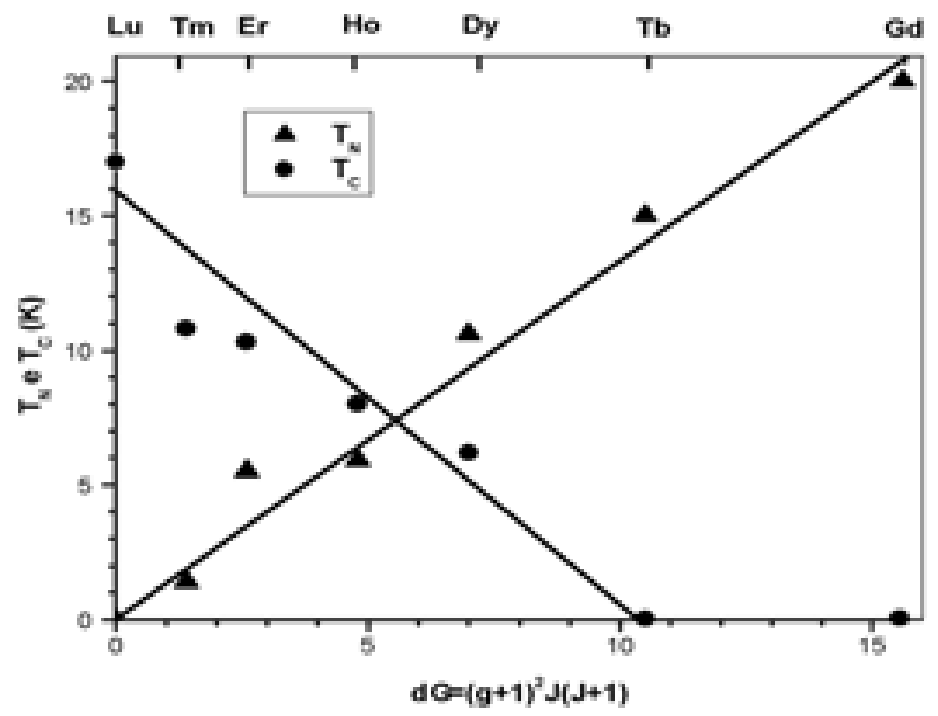

Figura 2.6: $T_{C}$ e $T_{N}$ em função do fator de de Gennes dos borocarbetos.

de desordem). Os borocarbetos quaternários foram classificados como supercondutores tipo onda-s convencional.

Com relação à esta simetria existe controvérsias, porém experimentos usando técnicas de de Haas-van Alphen ${ }^{32}$ parecem confirmar a classificação de ondas, com um gap da energia fortemente anisotrópico.

\subsection{Ordem magnética e supercondutividade nos borocarbetos}

Nesta seção são considerados os compostos $R N i_{2} B_{2} C$, onde os íons $R^{3+}$ têm camada $4 f$ incompleta. Os compostos com $R=\mathrm{Pr}, \mathrm{Nd}$, Gd e Tb só apresentam propriedades magnéticas, enquanto que $R=$ Dy, Ho, Er e Tm exibem coexistência de magnetismo e supercondutividade.

A fim de pesquisar o magnetismo $4 f$ nestes compostos várias medidas foram realizadas: espalhamento elástico ${ }^{33}$ e inelástico ${ }^{35}$ com nêutrons, relaxamento muon-spin ${ }^{36}$, efeito Mossbauer ${ }^{37,38}$, espalhamento de troca ressonante de raios-X $(\mathrm{XRES})^{39}$, magnetização e susceptibilidade magnética ${ }^{40}$, resistividade e ressonância magnética $(\mathrm{RM})^{41}$, calor especifico ${ }^{42,43}$, etc. Os valores relativamente grandes das temperaturas de ordenamento magnético $T_{m}$ ( $T_{N}$ e $T_{I C}$ dependendo se a ordem magnética é comensurável ou incomensurável respectivamente) e seu escalamento linear com o fator de de Gennes (vide figura 2.6) definido como:

$$
d G=\left(g_{J}-1\right)^{2} J(J+1)
$$


onde $g_{J}$ é o fator de Landé e $\mathrm{J}$ o momento angular total dos íons $R^{3+}$, indicam uma interação forte entre os momentos magnéticos $\mathrm{R}$, os quais são claramente dominados pela interação Ruderman-Kittel-KasuyaYosida(RKKY). Na tabela 2.2, são dadas alguns valores do fator de $d G$ para os ions livres $R^{3+}$. A estrutura magnética e em particular a direção local dos momentos magnéticos $\mathrm{R}$, é o resultado da competição entre a interação RKKY e o campo elétrico cristalino (CEF). Em geral estes compostos se ordenam antiferromagneticamente, porém em alguns casos $(\mathrm{R}=\mathrm{Tb}$ e $\mathrm{Er})$ se observa a presença de uma estrutura magnética tipo ferromagnetismo fraco ${ }^{44}$ (WFM), um pequeno momento magnético liquido, como resultado de desvios nos momentos magnéticos $\mathrm{R}$ ordenados antiferromagneticamente. No caso do Ho existem estruturas magnéticas adicionais a temperaturas altas, como veremos mais adiante. Para apenas um elétron ou um buraco na camada $4 f$ do íon $R^{3+}$, i.e. para o $\mathrm{R}=\mathrm{Ce}$ ou $\mathrm{Yb}$, efeitos de hibridização e correlação levam à supressão do magnetismo. Não se detectou momentos magnéticos $\operatorname{associados}^{33}$ ao Ni.

\subsection{Ordem magnética e CEF}

As estruturas magnéticas do estado fundamental de alguns borocarbetos são mostradas nas figuras 2.7 e 2.8. Estas estruturas são caracterizadas pelas magnitudes e direções dos momentos magnéticos em relação aos eixos cristalográficos, assim como dos momentos magnéticos $\mathrm{R}$ vizinhos. As magnitudes dos momentos magnéticos e o tipo de estruturas magnéticas, comensurável ou incomensurável, nestes compostos depende da competição entre a anisotropia de um só íon devido ao campo cristalino e a interação RKKY. A estrutura magnética é caracterizada por um vetor de propagação $\overrightarrow{\mathbf{q}}$, que pode ser comensurável ou não com um vetor da rede recíproca. A direção dos momentos magnéticos $\mathrm{R}$ são governadas pelo campo cristalino e a estrutura magnética incomensurável é um efeito típico da interação RKKY. Das figuras 2.7 e 2.8 vemos que há dois tipos de estruturas magnéticas: momentos magnéticos paralelos ou perpendiculares ao eixo $c$; e com relação ao eixo de fácil magnetização, eles podem ser paralelos ou antiparalelos a esse eixo. Contudo, em alguns casos se observou pequenos desvios a partir de seu alinhamento forte (paralelo ou antiparalelo). Como exemplo temos, a estrutura espiral e a estrutura modulada no eixo a observada no $\mathrm{HoNi}_{2} \mathrm{~B}_{2} \mathrm{C}$ a temperaturas altas ou ferromagnetismo fraco (WFM) no $\mathrm{TbNi}_{2} \mathrm{~B}_{2} C$ e no $E r N i_{2} B_{2} C$. Como a interação RKKY esta mediada pelos elétrons de condução, a estrutura de magnetização incomensurável depende também dos detalhes da estrutura eletrônica. Isto 
é por que o vetor de encaixamento perfeito $\vec{\tau}=(0.55,0,0)$ (a primeira componente em unidades de $\frac{2 \pi}{a}$ ) se manifesta como um vetor de onda da modulação de diferentes estruturas incomensuráveis como foi encontrado em vários compostos $R \mathrm{Ni}_{2} \mathrm{~B}_{2} \mathrm{C}$.

\subsection{Estrutura magnética para $R=\mathbf{G d}$, Ho, Er, Dy, Tb e Nd}

Nesta seção fazemos um resumo das estruturas magnéticas da série em estudo. Veremos que elas apresentam uma ampla variedade de estruturas magnéticas no estado fundamental em ausêscia de campo magnético externo.

\subsection{1 $G d N i_{2} B_{2} C$}

$\mathrm{O} G d N i_{2} B_{2} C$ se ordena magneticamente em uma estrutura de onda de densidade de spin (SDW) em $T_{N}=20 K$. A estrutura SDW é uma classe de estado tipo AF mas com uma densidade de spin formando uma onda estacionaria. Este composto também sofre uma segunda transição em $T_{R}=14 K$, que é atribuido a uma reorientação de spin. Abaixo de $T_{N}$ este composto encontra-se em um estado AF incomensurável, com vetor de onda da modulação próximo ao vetor de encaixamento perfeito. Entre $T_{N}$ e $T_{R}$ a estrutura magnética é mostrada na figura 2.8. Para $T<T_{R}$ os momentos magnéticos do Gd desenvolvem uma componente adicional ao longo do eixo $c$ e o vetor de onda de modulação aumenta até $\overrightarrow{\mathbf{q}}=(0.553,0,0)$ em $3.5 \mathrm{~K}$.

\subsection{2 $\mathrm{HoNi}_{2} \mathrm{~B}_{2} \mathrm{C}$}

Três tipos de ordem AF estão presentes no $\mathrm{HoNi}_{2} \mathrm{~B}_{2} \mathrm{C}$. A estrutura magnética AF comensurável, mostrada na figura 2.7(a), se forma em $T_{N}=$ $5.2 K$ com $\overrightarrow{\mathbf{q}}_{1}=(0,0,1)$ (a terceira componente em unidades de $\frac{2 \pi}{c}$ ). Adicionalmente, na faixa de temperatura entre $T_{N}<T<T_{C}$ há uma estrutura magnética espiral incomensurável ao longo do eixo a com vetor de onda de modulação $\overrightarrow{\mathbf{q}}_{2}=(0,0,0.916)$. Ainda outra estrutura magnética incomensurável ao longo do eixo $a$, esta presente, com vetor de onda de modulação $\overrightarrow{\mathbf{q}}_{3}=(0.58,0,0)$, próximo ao vetor de encaixamento perfeito dos borocarbetos supercondutores $L u N i_{2} B_{2} C$ e $Y N i_{2} B_{2} C$.

\subsection{3 $\mathrm{ErNi}_{2} \mathrm{~B}_{2} \mathrm{C}$}

O $\operatorname{Er} N i_{2} B_{2} C$ se ordena magneticamente em $T_{N}=6,8 \mathrm{~K}$ numa estrutura SDW transversal com um vetor de onda de modulação paralelo ao eixo $a$ e os momentos magnéticos paralelos ao eixo b (ou vice versa), ver figura $2.7(\mathrm{~b}) \mathrm{e}$ 2.7 (e). O vetor da modulação é $\overrightarrow{\mathbf{q}}=(0.553,0,0)$. Próximo a $2.3 \mathrm{~K}$ ocorre uma 


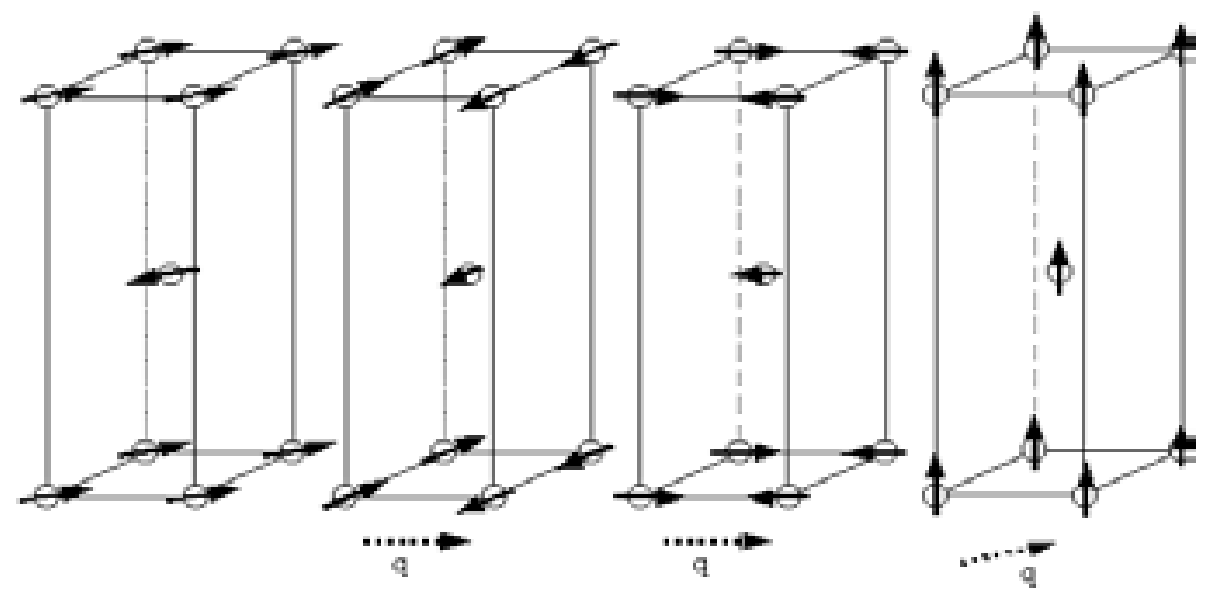

(a) Pr, Dy, Ho

(b) Er

(c) $\mathrm{Tb}$

(d) Tm
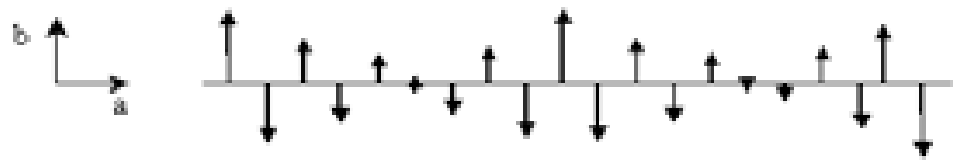

(e) $\mathrm{Er}_{\mathrm{N}} \mathrm{F}_{2} \mathrm{~B}_{2} \mathrm{C}$

Figura 2.7: Estruturas magnética do (a) Pr, Dy e Ho; (b) Er; (c) Tb; (d) Tm; (e) $E r N i_{2} B_{2} C$ mostrando sobre varias células unitarias ${ }^{5}$.

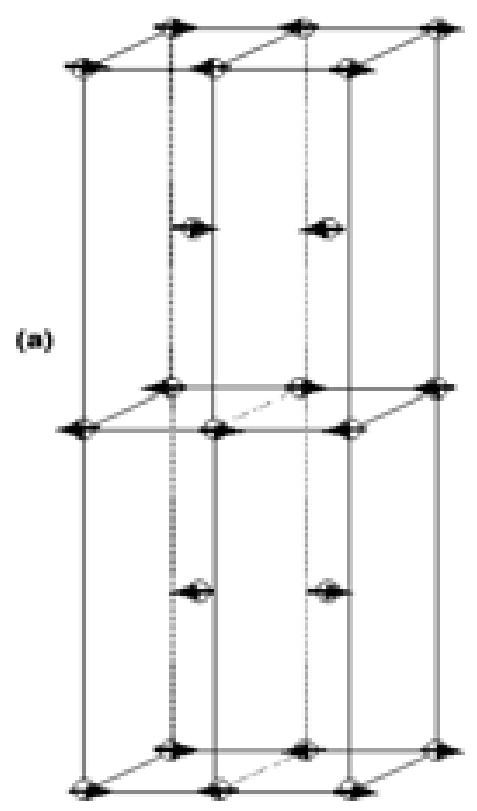

(b)

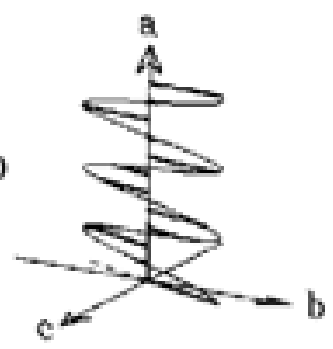

(c)

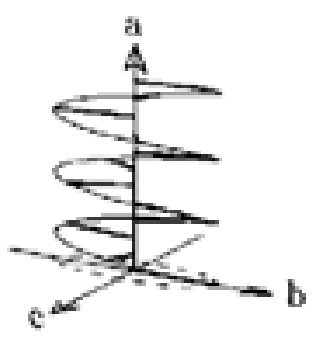

Figura 2.8: Estrutura magnética do: (a) $N d N i_{2} B_{2} C^{5}$, (b) e (c) $G d N i_{2} B_{2} C^{34}$. 
segunda transição de fase e o sistema entra em um estado ordenado WFM. Acredita-se que esta estrutura surge como consequência de um aumento de um aprisionamento dos vórtices (flux pinning) a baixas temperaturas ${ }^{12}$.

\begin{tabular}{||c|c|c|c|c||}
\hline \hline Composto & Estrutura magética & Direção dos Momentos & Vetor de onda & $\Delta(K)$ \\
\hline$N d N i_{2} B_{2} \mathrm{C}$ & $\mathrm{AFM}$ & {$[100]$} & $(0.5,0,0.5)$ & 0 \\
\hline$G d N i_{2} B_{2} \mathrm{C}$ & $\mathrm{SDW}$ & {$[010]$} & $(0.55,0,0)$ & 0 \\
\hline $\mathrm{TbNi} i_{2} B_{2} \mathrm{C}$ & $\mathrm{SDW} / W F M$ & {$[100]$} & $(0.555,0,0)$ & 8.9 \\
\hline $\mathrm{DyN} i_{2} B_{2} \mathrm{C}$ & $\mathrm{AFM}$ & {$[110]$} & $(0,0,1)$ & 0 \\
\hline $\mathrm{HoNi} i_{2} B_{2} \mathrm{C}$ & $\mathrm{AFM}$ & {$[110]$} & $(0,0,1)$ & 8.5 \\
\hline$E r N i_{2} B_{2} \mathrm{C}$ & $\mathrm{SDW} / W F M$ & {$[010]$} & $(0.5526,0,0)$ & $0,5.4$ \\
\hline $\mathrm{TmNi} i_{2} B_{2} \mathrm{C}$ & $\mathrm{SDW}$ & {$[001]$} & $(0.093 .0 .093,0)$ & 0 \\
\hline \hline
\end{tabular}

Tabela 2.3: Tipos de estrutura magnética e parâmetros a campo magnético nulo para alguns compostos da serie $R N i_{2} B_{2} C$. $\Delta$ é o gap do espectro de magnons em unidades de grau Kelvin ${ }^{12}$.

\subsection{4 $\mathrm{DyNi}_{2} \mathrm{~B}_{2} \mathrm{C}$}

Este composto é o único membro da serie $R N i_{2} B_{2} C$, na qual o estado supercondutor ocorre depois do composto ter entrado no estado AF, da figura 2.6 vemos que $T_{C}<T_{N}$, por esta razão o composto é às vêzes chamado de magneto supercondutor. A estrutura magnética do estado fundamental é mostrada na figura 2.7(a), e consiste em planos ferromagnéticos (FM), com momentos magnéticos orientados na direção [110] e os planos adjacentes odenados antiferromagneticamente.

\subsection{5 $\mathrm{TbNi}_{2} \mathrm{~B}_{2} \mathrm{C}$}

A estrutura magnética (fig. 2.7c) do $\mathrm{TbNi} i_{2} \mathrm{~B}_{2} \mathrm{C}$ é SDW incomensurável ao longo do eixo a com momentos magnéticos paralelos ao eixo a $\left(T_{N}=\right.$ $14.5 K)$. O vetor de onda de modulação é $\overrightarrow{\mathbf{q}}=(0.55,0,0)$ e praticamente coincide com o vetor de encaixamento perfeito dos supercondutores magnéticos. Uma transição ao estado WFM ocorre em $T_{W F M}=8 \mathrm{~K}$ e uma transição de reorientação de spin em $T_{R}=5 \mathrm{~K}$.

\subsection{6 $N d N i_{2} B_{2} C$}

Este composto é um supercondutor AF, com estrutura magnética mostrada na figura 2.8. Os momentos magnéticos se encontram na direção do eixo $a$ e seu acoplamento é AF nesta direção, enquanto que na direção $b$ o acoplamento é FM. Ao longo do eixo $c$ um primeiro vizinho acima está 
alinhado antiparalelamente e outro abaixo está alinhado paralelamente.

\subsection{Hipótese básica}

A física dos borocarbetos é caracterizada pela presença de duas espécies distintas de elétrons:

i) os elétrons $4 f$ das terras raras e

ii) os elétrons de condução do Ni.

Como já nos referimos, as terras raras são as responsáveis pela ordem magnética enquanto os elétrons do metal de transição são os responsáveis pela supercondutividade. No presente trabalho adotamos o ponto de vista de que a ordem magnética quando se encontra dentro da fase supercondutora não é afetado por esta fase, e assim pode ser descrita por um hamiltoniano de spin (com normalização eventual dos parâmetros). O fato da ordem magnética da família dos supercondutores (Ho, Dy) e dos não supercondutores (Gd, Pr) serem similares, sustenta esta hipótese. 


\section{3}

\section{Aproximação de ondas de spin}

\subsection{O hamiltoniano}

Da estrutura magnética dos borocarbetos (Pr, Dy, Ho), descrita na seção anterior, observamos que podemos usar como ponto de partida para a teoria de ondas de spin na fase antiferromagnética (AF) o modelo de Neel de duas subredes. Nestes compostos a estrutura magnética é tal que dentro de uma camada o acoplamento é ferromagnético $(\mathrm{FM})\left(J_{1}>0\right)$ porém entre camadas adjacentes o acopamento é $\mathrm{AF}\left(-J_{2}, J_{2}>0\right)$.

O hamiltoniano é então (com os operadores de spin em unidades de $\hbar$ ):

$$
\begin{aligned}
\mathcal{H}= & -\sum_{<i, j>\in A} J_{1} \vec{S}_{i} \cdot \vec{S}_{j}-\sum_{<i, j>\in B} J_{1} \vec{S}_{i} \cdot \vec{S}_{j}+\sum_{<i, j>\in A B} J_{2} \vec{S}_{i}^{A} \cdot \vec{S}_{j}^{B} \\
& -2 \mu_{0}\left(\vec{H}_{e x t}+\vec{H}_{A}\right) \sum_{i \in A} \vec{S}_{i}-2 \mu_{0}\left(\vec{H}_{e x t}-\vec{H}_{A}\right) \sum_{j \in B} \vec{S}_{j}
\end{aligned}
$$

onde $\langle i, j\rangle$ indica soma sobre pares de primeiros vizinhos. O primeiro termo descreve a interação na subrede A; o segundo termo descreve a interação na subrede $\mathrm{B}$, enquanto o terceiro corresponde à interação entre camadas vizinhas. Cada subrede contem $\mathrm{N}$ sitios. $\vec{H}_{A}$ é um campo de anisotropia efetivo simulando o efeito do campo elétrico cristalino (CEF), que força os momentos magnéticos a se orientar numa direção particular, que é a direção da magnetização na subrede A. Portanto, na subrede B o campo de anisotropia é $-\vec{H}_{A}$. Esta descrição é apropriada, por exemplo, para os compostos contendo Ho ou Dy ou Pr. $\vec{H}_{e x t}$ é um campo magnético externo aplicado, e $\mu_{0}=\frac{1}{2} g_{J} \mu_{B}$, onde $g_{J}$ é o fator de Landé, cujos valores são dados na tabela 2.2 e $\mu_{B}$ é o magneton de Bohr.

Transformamos o hamiltoniano, equação (3.1), aplicando a teoria de ondas de $\operatorname{spin}^{45}$, com o campo de anisotropia $\vec{H}_{A}$ e o campo magnético externo uniforme $\vec{H}_{e x t}$ na direção [110].

\subsection{Cálculo da relação de dispersão dos borocarbetos}

Aplicando a transformação de Holstein-Primakoff ${ }^{45}$, expressamos os operadores de spin da subrede A na forma ( $\mathrm{S}$ é o número quântico do momento angular total):

$$
S_{A, l}^{+}=(2 S)^{1 / 2} f_{l}(S) a_{l}
$$




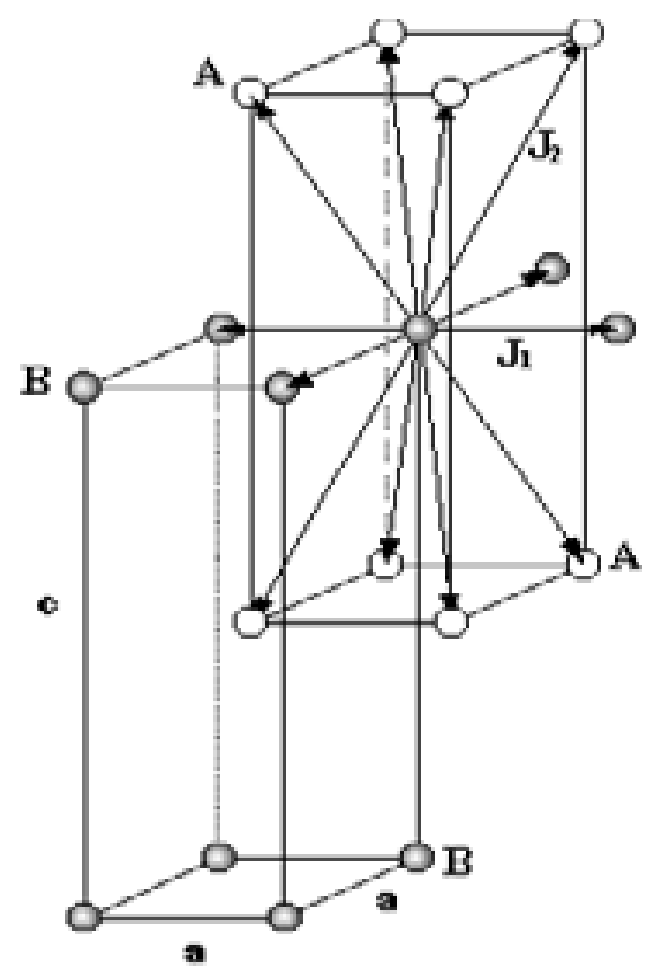

Figura 3.1: Ilustração da divisão em subredes no hamiltoniano (3.1).

$$
\begin{aligned}
& S_{A, l}^{-}=(2 S)^{1 / 2} a_{l}^{+} f_{l}(S) \\
& S_{A, l}^{z}=S-a_{l}^{+} a_{l}
\end{aligned}
$$

enquanto que os da subrede B tem a forma:

$$
\begin{aligned}
& S_{B, m}^{+}=(2 S)^{1 / 2} b_{m}^{+} f_{m}(S) \\
& S_{B, m}^{-}=(2 S)^{1 / 2} f_{m}(S) b_{m} \\
& S_{B, m}^{z}=-S+b_{m}^{+} b_{m}
\end{aligned}
$$

com

$$
f_{i}(S)=\left[1-\frac{n_{i}}{2 S}\right]^{1 / 2}
$$

onde $a_{l}^{+}\left(a_{l}\right)$ e $b_{m}^{+}\left(b_{m}\right)$ são os operadores de criação (aniquilação) de bosons no l-ésimo sitio da subrede A e no $m$-ésimo sitio da subrede B respectivamente; e $n_{l}=a_{l}^{+} a_{l}$ (subrede A) ou $n_{m}=b_{m}^{+} b_{m}$ (subrede B) são os operadores números de ocupação.

A direção de quantização (z) é a direção [110]. A aproximação linear consiste em tomar apenas o primeiro termo da expansão de (3.4); os demais dariam origem a interações entre os magnons, que não serão consideradas neste 
trabalho.

Agora introduzimos as transformadas de Fourier dos operadores $a_{l}^{+}\left(a_{l}\right)$ e $b_{m}^{+}$ $\left(b_{m}\right)$ :

$$
\begin{array}{cc}
c_{\vec{k}}=\frac{1}{N^{1 / 2}} \sum_{l} e^{i \vec{k} \cdot \vec{x}_{l}} a_{l} ; & c_{\vec{k}}^{+}=\frac{1}{N^{1 / 2}} \sum_{l} e^{-i \vec{k} \cdot \vec{x}_{l}} a_{l}^{+} \\
d_{\vec{k}}=\frac{1}{N^{1 / 2}} \sum_{m} e^{-i \vec{k} \cdot \vec{x}_{m}} b_{m} ; & d_{\vec{k}}^{+}=\frac{1}{N^{1 / 2}} \sum_{m} e^{i \vec{k} \cdot \vec{x}_{m}} b_{m}^{+}
\end{array}
$$

onde a soma é sobre os $\mathrm{N}$ átomos da subrede correspondente.

Então:

$$
\begin{gathered}
S_{A, l}^{+}=\left(\frac{2 S}{N}\right)^{1 / 2}\left[\sum_{\vec{k}} e^{-i \vec{k} \cdot \vec{x}_{l}} c_{\vec{k}}+\cdots\right] \\
S_{A, l}^{-}=\left(\frac{2 S}{N}\right)^{1 / 2}\left[\sum_{\vec{k}} e^{i \vec{k} \cdot \vec{x}_{l}} c_{\vec{k}}^{+}+\cdots\right] \\
S_{A, l}^{z}=S-\frac{1}{N} \sum_{\vec{k}, \vec{k}^{\prime}} e^{i\left(\vec{k}-\vec{k}^{\prime}\right) \cdot \vec{x}_{l}} c_{\vec{k}}^{+} c_{\overrightarrow{k^{\prime}}} \\
S_{B, m}^{+}=\left(\frac{2 S}{N}\right)^{1 / 2}\left[\sum_{\vec{k}} e^{-i \vec{k} \cdot \vec{x}_{m}} d_{\vec{k}}^{+}+\cdots\right] \\
S_{B, m}^{-}=\left(\frac{2 S}{N}\right)^{1 / 2}\left[\sum_{\vec{k}} e^{i \vec{k} \cdot \vec{x}_{m}} d_{\vec{k}}+\cdots\right] \\
S_{B, m}^{z}=-S+\frac{1}{N} \sum_{\vec{k}, \vec{k}^{\prime}} e^{-i\left(\vec{k}-\vec{k}^{\prime}\right) \cdot \vec{x}_{m}} d_{\vec{k}}^{+} d_{\overrightarrow{k^{\prime}}}
\end{gathered}
$$

Então, substituindo as equações (3.7) e (3.8) em (3.1) o hamiltoniano transformado nas novas variáveis de spin é:

$$
\mathcal{H}=\mathcal{H}_{0}^{\prime}+\mathcal{H}_{1}
$$

com

$$
\mathcal{H}_{0}^{\prime}=-\left(z_{1} J_{1}+z_{2} J_{2}\right) N S^{2}-4 \mu_{0} H_{A} N S-z_{1} J_{1} S \sum_{\vec{k}} \gamma_{\vec{k}}^{(1)}
$$




$$
\begin{aligned}
\mathcal{H}_{1}= & \sum_{\vec{k}}\left[z_{1} J_{1} S\left(1-\gamma_{\vec{k}}^{(1)}\right)+z_{2} J_{2} S+2 \mu_{0} H_{A}\right]\left(c_{\vec{k}}^{+} c_{\vec{k}}+d_{\vec{k}}^{+} d_{\vec{k}}\right) \\
& +\sum_{\vec{k}} z_{2} J_{2} S \gamma_{\vec{k}}^{(2)}\left(c_{\vec{k}} d_{\vec{k}}+c_{\vec{k}}^{+} d_{\vec{k}}^{+}\right)+2 \mu_{0} H_{\text {ext }} \sum_{\vec{k}}\left(c_{\vec{k}}^{+} c_{\vec{k}}-d_{\vec{k}}^{+} d_{\vec{k}}\right)
\end{aligned}
$$

onde:

$$
\begin{aligned}
& \gamma_{\vec{k}}^{(1)}=\frac{1}{z_{1}} \sum_{\vec{\delta}_{1}} e^{i \vec{k} \cdot \vec{\delta}_{1}} \\
& \gamma_{\vec{k}}^{(2)}=\frac{1}{z_{2}} \sum_{\vec{\delta}_{2}} e^{i \vec{k} \cdot \vec{\delta}_{2}}
\end{aligned}
$$

A soma sobre $\vec{\delta}_{1}$ é sobre os $z_{1}=4$ primeiros vizinhos que se encontram no mesmo plano (A ou B), enquanto que a sobre $\vec{\delta}_{2}$ é sobre os $z_{2}=8$ primeiros vizinhos da outra subrede. Na rede original a soma sobre $\vec{\delta}_{2}$ corresponde a terceiros vizinhos. Estes vetores são:

$$
\vec{\delta}_{1}=\left\{\begin{array}{l} 
\pm a \hat{\mathbf{x}} \\
\pm a \hat{\mathbf{y}}
\end{array} \quad \vec{\delta}_{2}=\left\{\begin{array}{c} 
\pm\left(\frac{a}{2} \hat{\mathbf{x}}+\frac{a}{2} \hat{\mathbf{y}}+\frac{c}{2} \hat{\mathbf{z}}\right) \\
\pm\left(-\frac{a}{2} \hat{\mathbf{x}}+\frac{a}{2} \hat{\mathbf{y}}+\frac{c}{2} \hat{\mathbf{z}}\right) \\
\pm\left(\frac{a}{2} \hat{\mathbf{x}}-\frac{a}{2} \hat{\mathbf{y}}+\frac{c}{2} \hat{\mathbf{z}}\right) \\
\pm\left(\frac{a}{2} \hat{\mathbf{X}}+\frac{a}{2} \hat{\mathbf{y}}-\frac{c}{2} \hat{\mathbf{z}}\right)
\end{array}\right.\right.
$$

Substituindo estes vetores posição nas equações (3.12) e (3.13) obtemos:

$$
\begin{gathered}
\gamma_{\vec{k}}^{(1)}=\frac{1}{2}\left[\cos \left(a k_{x}\right)+\cos \left(a k_{y}\right)\right] \\
\gamma_{\vec{k}}^{(2)}=\frac{1}{4}\left[\cos \left(\frac{a}{2} k_{x}+\frac{a}{2} k_{y}+\frac{c}{2} k_{z}\right)+\cos \left(\frac{a}{2} k_{x}+\frac{a}{2} k_{y}-\frac{c}{2} k_{z}\right)\right. \\
\left.+\cos \left(-\frac{a}{2} k_{x}+\frac{a}{2} k_{y}+\frac{c}{2} k_{z}\right)+\cos \left(\frac{a}{2} k_{x}-\frac{a}{2} k_{y}+\frac{c}{2} k_{z}\right)\right]
\end{gathered}
$$

Seja:

$$
\begin{aligned}
A_{k} & =z_{1} J_{1} S\left(1-\gamma_{\vec{k}}^{(1)}\right)+z_{2} J_{2} S+2 \mu_{0} H_{A} \\
& =4 J_{1} S\left\{1-\frac{1}{2}\left[\cos \left(a k_{x}\right)+\cos \left(a k_{y}\right)\right]\right\}+8 J_{2} S+2 \mu_{0} H_{A}
\end{aligned}
$$

e

$$
\begin{aligned}
B_{k}= & z_{2} J_{2} S \gamma_{\vec{k}}^{(2)} \\
= & 2 J_{2} S\left[\cos \left(\frac{a}{2} k_{x}+\frac{a}{2} k_{y}+\frac{c}{2} k_{z}\right)+\cos \left(\frac{a}{2} k_{x}+\frac{a}{2} k_{y}-\frac{c}{2} k_{z}\right)\right. \\
& \left.+\cos \left(-\frac{a}{2} k_{x}+\frac{a}{2} k_{y}+\frac{c}{2} k_{z}\right)+\cos \left(\frac{a}{2} k_{x}-\frac{a}{2} k_{y}+\frac{c}{2} k_{z}\right)\right]
\end{aligned}
$$

Então podemos escrever: 


$$
\begin{aligned}
\mathcal{H}_{1}= & +\sum_{\vec{k}} A_{k}\left(c_{\vec{k}}^{+} c_{\vec{k}}+d_{\vec{k}}^{+} d_{\vec{k}}\right)+\sum_{\vec{k}} B_{k}\left(c_{\vec{k}} d_{\vec{k}}+c_{\vec{k}}^{+} d_{\vec{k}}^{+}\right) \\
& +2 \mu_{0} H_{\text {ext }} \sum_{\vec{k}}\left(c_{\vec{k}}^{+} c_{\vec{k}}-d_{\vec{k}}^{+} d_{\vec{k}}\right)
\end{aligned}
$$

A expressão (3.19), embora quadrática nos operadores bosônicos, ainda não tem a forma diagonalizada. Para isso introduzimos a transformação (canônica) de Bogoliubov ${ }^{45}$ :

$$
\begin{array}{ll}
\alpha_{k}=u_{k} c_{k}-v_{k} d_{k}^{+} ; & \alpha_{k}^{+}=u_{k} c_{k}^{+}-v_{k} d_{k} \\
\beta_{k}=u_{k} d_{k}-v_{k} c_{k}^{+} ; & \beta_{k}^{+}=u_{k} d_{k}^{+}-v_{k} c_{k}
\end{array}
$$

onde $u_{k}$ e $v_{k}$ são funções reais. Para manter a relação de comutação, devemos ter:

$$
u_{k}^{2}-v_{k}^{2}=1
$$

Impondo que (3.20) e (3.21) diagonalizem o hamiltoniano e definindo:

$$
u_{k}=\cosh \frac{1}{2} \Theta_{k} ; \quad v_{k}=\sinh \frac{1}{2} \Theta_{k}
$$

obtemos:

$$
\tanh \Theta_{k}=-\frac{B_{k}}{A_{k}}
$$

Assim

$$
\mathcal{H}=\mathcal{H}^{\prime \prime}{ }_{0}+\mathcal{H}_{1}^{\prime}
$$

com (em unidades em que $\hbar=1$ )

$$
\begin{aligned}
& \mathcal{H}_{0}^{\prime \prime}=-4 J_{1} N S(S+1)-8 J_{2} N S(S+1)-4 \mu_{0} H_{A} N(S+1 / 2) \\
& \mathcal{H}_{1}^{\prime}=\sum_{k} \omega_{k}^{+}\left(n_{k}^{+}+\frac{1}{2}\right)+\sum_{k} \omega_{k}^{-}\left(n_{k}^{-}+\frac{1}{2}\right)
\end{aligned}
$$

onde:

$$
\omega_{k}^{ \pm}=\omega_{k} \pm 2 \mu_{0} H_{e x t}
$$

$\mathrm{e}$

$$
\omega_{k}=\left(A_{k}^{2}-B_{k}^{2}\right)^{1 / 2}
$$

$\mathrm{e}$

$$
n_{k}^{+}=\alpha_{k}^{+} \alpha_{k} ; \quad n_{k}^{-}=\beta_{k}^{+} \beta_{k}
$$




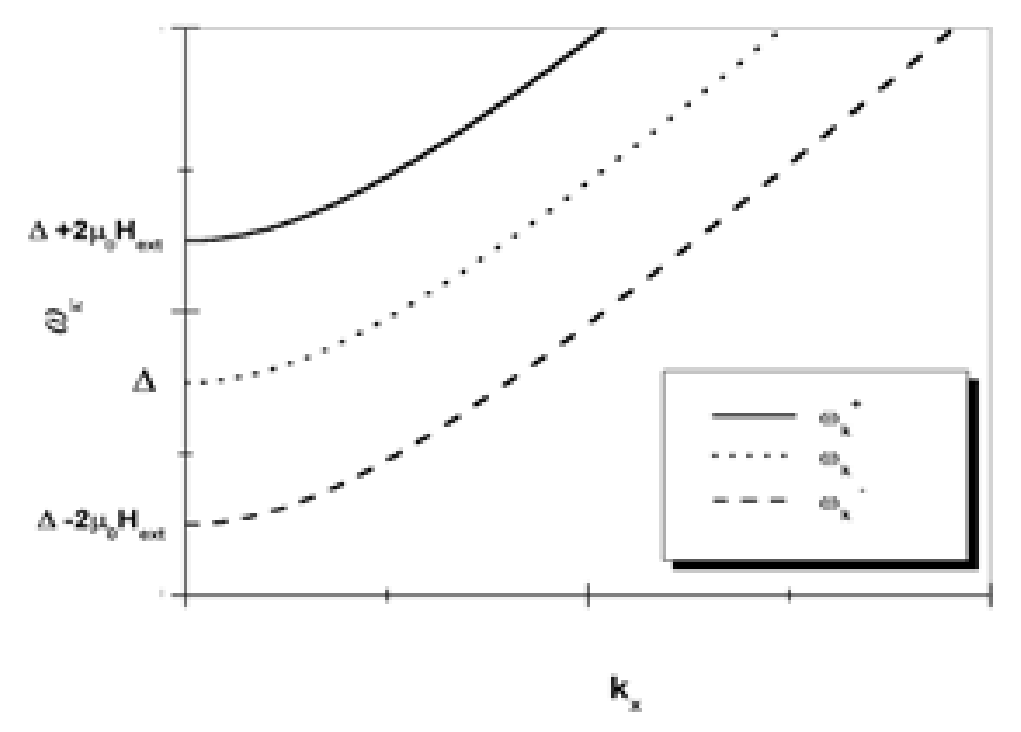

Figura 3.2: Espectro de energia das ondas de spin na fase AF com campo magnético aplicado.

O espectro de energia na presença de um campo magnético externo apresenta dois ramos, um associado ao operador $\alpha$ e ou outro ao operador $\beta . n_{k}^{ \pm}$são os operadores números de ocupação, dos respectivos modos e suas médias termodinâmicas são as funções distribuição de Bose-Einsten.

Para o caso em que o campo magético externo é nulo $\left(H_{e x t}=0\right)$, obviamente, o espectro é degenerado. Observemos que o campo magnético introduz deslocamentos rigidos, independente de $\mathrm{k}$, no ramo correspondente a $H_{\text {ext }}=0$ Das equações (3.17), (3.18) e (3.28), vemos que cada ramo do espectro apresenta um gap igual a:

$$
\begin{aligned}
\omega_{k=0}^{ \pm} \equiv \Delta^{ \pm}\left(H_{e x t}\right) & =\left[32 J_{2} S \mu_{0} H_{A}+\left(2 \mu_{0} H_{A}\right)^{2}\right]^{1 / 2} \pm 2 \mu_{0} H_{e x t} \\
& =\Delta \pm 2 \mu_{0} H_{e x t}
\end{aligned}
$$

onde

$$
\Delta \equiv \Delta^{ \pm}\left(H_{e x t}=0\right)=\left[32 J_{2} S \mu_{0} H_{A}+\left(2 \mu_{0} H_{A}\right)^{2}\right]^{1 / 2}
$$

( Na presença do campo magnético o gap verdadeiro é $\Delta^{-}\left(H_{e x t}\right)$ ). Note que o gap $\Delta$ depende apenas de $J_{2}$ e $H_{A}$.

A baixas temperaturas os estados que mais contribuem para as propriedades físicas do sistema são os de energia mais baixa, nas vizinhanças de $k \simeq 0$. É justificável desenvolver em serie os cossenos nas equações (3.15) e (3.16) e manter termos até segunda ordem em k. Então

$$
\gamma_{\vec{k}}^{(1)} \cong \frac{1}{2}\left[2-\frac{1}{2} a^{2}\left(k_{x}^{2}+k_{y}^{2}\right)\right]
$$

e 


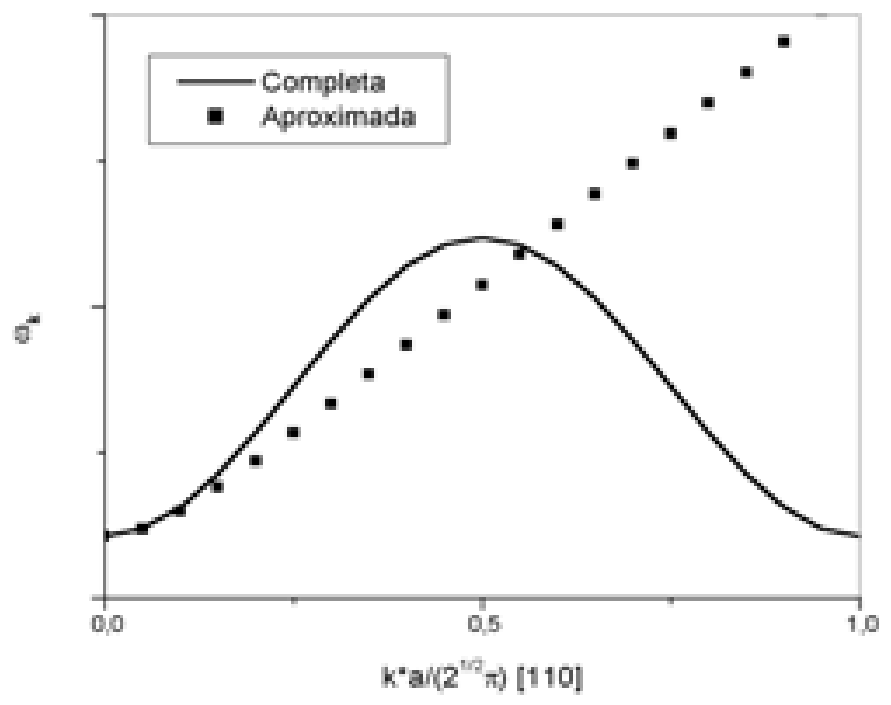

Figura 3.3: Relação de dispersão completa (3.29) e aproximada (3.36) das ondas de spin.

$$
\gamma_{\vec{k}}^{(2)} \cong \frac{1}{4}\left[4-\frac{1}{2} a^{2}\left(k_{x}^{2}+k_{y}^{2}\right)-\frac{1}{2} c^{2} k_{z}^{2}\right]
$$

Nesta aproximação de comprimentos de onda grandes, a relação de dispersão se escreve como a equação 3.28 (ver também figura 3.3) com

$$
\omega_{k}=\left[\Delta^{2}+c_{1} a^{2}\left(k_{x}^{2}+k_{y}^{2}\right)+c_{2} c^{2} k_{z}^{2}\right]^{1 / 2}
$$

onde $c_{1}$ e $c_{2}$ são:

$$
\begin{aligned}
& c_{1}=16\left(J_{1}+J_{2}\right) J_{2} S^{2}+\left(2 J_{1} S\right)\left(2 \mu_{0} H_{A}\right) \\
& c_{2}=16 J_{2}^{2} S^{2}
\end{aligned}
$$

Vemos que a equação (3.36) representa o espectro das ondas de spin de um sistema com simetria tetragonal.

\subsection{Densidade de estados}

A densidade de estados a campo magnético externo nulo, para cada banda, é definida pela relação:

$$
\mathcal{D}(\varepsilon)=\frac{V}{(2 \pi)^{3}} \int \frac{d S_{\varepsilon}}{\left|\nabla_{\vec{k}} \varepsilon\right|}
$$

onde $\varepsilon \equiv \omega_{\vec{k}}$ é dada pela equação (3.36), V é o volume do sistema e $d S_{\varepsilon}$ é o elemento de área no espaço $\mathrm{k}$ da superficie de energia constante e igual a $\varepsilon$. Da relação de dispersão (3.36) encontramos:

$$
\left|\nabla_{\vec{k}} \varepsilon\right|=\frac{1}{\varepsilon}\left[c_{1}\left(a^{2} c_{1}-c^{2} c_{2}\right) a^{2}\left(k_{x}^{2}+k_{y}^{2}\right)+c^{2} c_{2}\left(\varepsilon^{2}-\Delta^{2}\right)\right]^{1 / 2}
$$




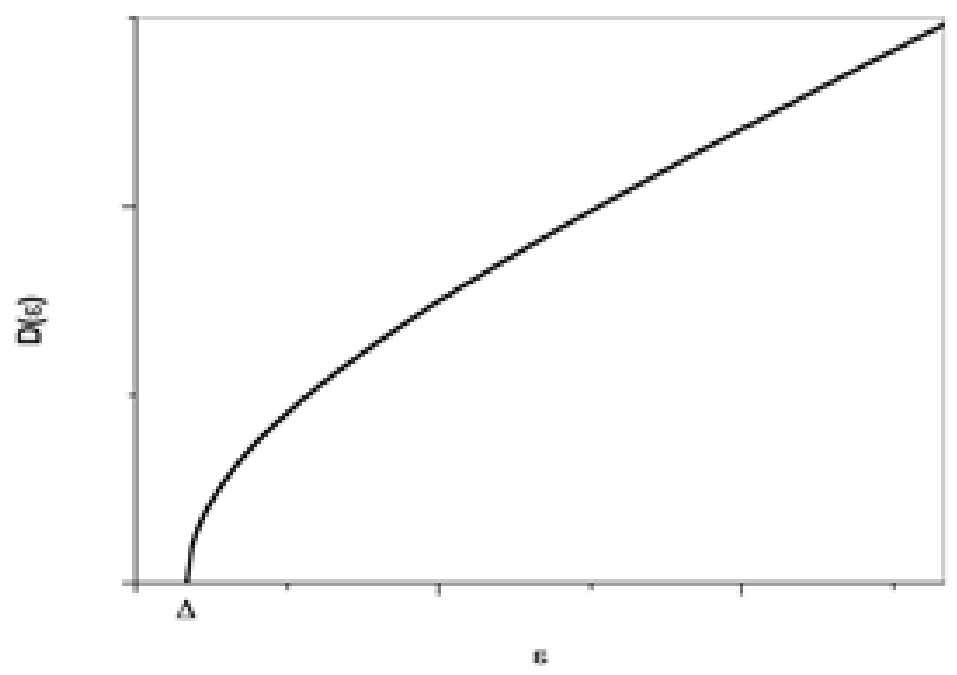

Figura 3.4: Densidade de estados em função da energia.

Dado que o elemento de superficie de energia constante pode ser escrito como:

$$
d S_{\varepsilon}=\sqrt{1+\left(\frac{\partial k_{z}}{\partial k_{x}}\right)^{2}+\left(\frac{\partial k_{z}}{\partial k_{y}}\right)^{2}} d k_{x} d k_{y}
$$

escrevemos $k_{z}$ de (3.36) como função de $k_{x}, k_{y}$ e $\varepsilon$ obtemos

$$
k_{z}=\frac{1}{c \sqrt{c_{2}}}\left[\varepsilon^{2}-\Delta^{2}-c_{1} a^{2}\left(k_{x}^{2}+k_{y}^{2}\right)\right]^{1 / 2}
$$

e

$$
d S_{\varepsilon}=\frac{\left[c^{2} c_{2}\left(\varepsilon^{2}-\Delta^{2}\right)+c_{1}\left(a^{2} c_{1}-c^{2} c_{2}\right) a^{2}\left(k_{x}^{2}+k_{y}^{2}\right)\right]^{1 / 2}}{c \sqrt{c_{2}}\left[\varepsilon^{2}-\Delta^{2}-c_{1} a^{2}\left(k_{x}^{2}+k_{y}^{2}\right)\right]^{1 / 2}} d k_{x} d k_{y}
$$

Então

$$
\int \frac{d S_{\varepsilon}}{\left|\nabla_{\vec{k}} \varepsilon_{\vec{k}}\right|}=\frac{4 \pi \varepsilon}{c \sqrt{c_{2}}} \int_{0}^{\Lambda} \frac{k d k}{\sqrt{\varepsilon^{2}-\Delta^{2}-c_{1} a^{2} k^{2}}}
$$

onde $k=\sqrt{k_{x}^{2}+k_{y}^{2}}$ e $\Lambda=\frac{\sqrt{\varepsilon^{2}-\Delta^{2}}}{a \sqrt{c_{1}}}$. Supuzemos que as superfícies de energias constantes são elipsoides de revolução.

Finalmente,

$$
\mathcal{D}(\varepsilon)=\frac{V}{2 \pi^{2} c_{1} \sqrt{c_{2}} a^{2} c} \varepsilon\left[\varepsilon^{2}-\Delta^{2}\right]^{1 / 2}
$$

Da definição do volume do sistema:

$$
V=2 N v_{c}=\nu N_{a} v_{c}
$$


onde $\mathrm{N}$ é o número de átomos TR em uma subrede, $v_{c}=\frac{a^{2} c}{2}$ é o volume da célula, $\nu$ é o número de moles e $N_{a}$ o número de Avogadro. Expressemos os parâmetros $c_{1}, c_{2}$ e a energia $\varepsilon$ em unidades de temperatura absoluta, o que fornece um fator $k_{B}$ que combinado com $N_{a}$ dá $\mathrm{R}\left(=8.3134 \frac{\mathrm{J}}{\mathrm{Kmol}}\right)$, a constante universal dos gases. Então escrevemos a densidade de estados para cada banda na ausência de campo magnético aplicado como $\nu \mathcal{D}(\varepsilon)$ onde:

$$
\mathcal{D}(\varepsilon)=\frac{R}{4 \pi^{2} c_{1} \sqrt{c_{2}}} \varepsilon\left[\varepsilon^{2}-\Delta^{2}\right]^{1 / 2}
$$

Observemos que em (3.47) $c_{1}$ e $c_{2}$, entram apenas como um fator geral multiplicativo. O modelo introduz três constantes $\left(J_{1}, J_{2}\right.$ e $\left.H_{A}\right) ; \Delta$ será tomado como um parâmetro fornecido pela experiência. 


\section{4 \\ Cálculo do calor específico em função da temperatura}

\subsection{Energia interna}

A contribuição à energia interna das excitações das ondas de spin, em equilíbrio térmico é dada por:

$$
E=E_{0}+2 \sum_{\vec{k}} \omega_{\vec{k}}\left\langle n_{\vec{k}}\right\rangle
$$

$\mathrm{Ou}$

$$
E=E_{0}+2 \int_{\Delta}^{\infty} \varepsilon \mathcal{D}(\varepsilon)\left\langle n_{\varepsilon}\right\rangle d \varepsilon
$$

onde $E_{0}$ é uma constante que independe da temperatura, correspondente à parte constante do hamiltoniano, o fator 2 é devido ao fato de que em ausência de campo magnético aplicado os ramos são degenerados e $\left\langle n_{\varepsilon}\right\rangle$ é a médias térmica do número de ocupação, dada pela distribuição de Bose-Einstein:

$$
\left\langle n_{\varepsilon}\right\rangle=\frac{1}{e^{\beta \varepsilon}-1}
$$

$\operatorname{com} \beta=\frac{1}{k_{B} T}$.

A integral foi estendida ao $\infty$, já que a contribuição de $\varepsilon$ grande é desprezível devido ao termo exponencial no denominador.

Substituindo a equação (3.47) em (4.2), temos:

$$
E=E_{0}+\frac{\nu R}{2 \pi^{2} c_{1} \sqrt{c_{2}}} \int_{\Delta}^{\infty} \frac{\varepsilon^{2}\left[\varepsilon^{2}-\Delta^{2}\right]^{1 / 2}}{e^{\frac{\varepsilon}{k_{B} T}}-1} d \varepsilon
$$

\subsection{Calor específico na fase AF 3D}

O calor específico molar $c_{m}$ é definido por:

$$
c_{m}=\frac{1}{\nu} \frac{d E}{d T}
$$

onde $\nu$ é o número de moles e a energia interna total $\mathrm{E}$ é dada pela equação (4.2).

De (4.2) e (4.5) o calor específico em função da temperatura a campo magnético externo nulo fica

$$
c_{m}\left(T, H_{e x t}=0\right)=\frac{1}{T^{2}} \int_{\Delta}^{\infty} \frac{\varepsilon^{2} \mathcal{D}(\varepsilon) e^{\beta \varepsilon}}{\left(e^{\beta \varepsilon}-1\right)^{2}} d \varepsilon
$$


Antes de integrar esta equação, escrevemos o fator $\frac{1}{\left(e^{\beta \varepsilon}-1\right)^{2}}$ da seguinte forma:

$$
\frac{1}{\left(e^{\beta \varepsilon}-1\right)^{2}}=\frac{e^{-2 \beta \varepsilon}}{\left(1-e^{-\beta \varepsilon}\right)^{2}}
$$

e desenvolvemos o fator $\frac{1}{\left(1-e^{-\beta \varepsilon}\right)^{2}}$ em serie de potências, usando a relação:

$$
\frac{1}{(1-x)^{2}}=\sum_{n=0}^{\infty}(n+1) x^{n}
$$

obtendo:

$$
\frac{1}{\left(e^{\beta \varepsilon}-1\right)^{2}}=\sum_{n=0}^{\infty}(n+1) e^{-(n+2) \beta \varepsilon}
$$

Então, substituindo a equação anterior, (4.6) se pode expressar como:

$$
c_{m}\left(T, H_{e x t}=0\right)=\frac{1}{T^{2}} \sum_{n=1}^{\infty} n \int_{\Delta}^{\infty} \varepsilon^{2} \mathcal{D}(\varepsilon) e^{-n \beta \varepsilon} d \varepsilon
$$

Substituindo a densidade de estados, equação (3.47), na equação anterior obtemos:

$$
c_{m}\left(T, H_{e x t}=0\right)=\frac{R}{2 \pi^{2} c_{1} \sqrt{c_{2}}} \frac{1}{T^{2}} \sum_{n=1}^{\infty} I_{n}
$$

com

$$
I_{n}=n \int_{\Delta}^{\infty} \varepsilon^{3}\left[\varepsilon^{2}-\Delta^{2}\right]^{1 / 2} e^{-n \beta \varepsilon} d \varepsilon
$$

Fazendo a mudança de variável :

$$
\begin{gathered}
\varepsilon=\Delta \cosh (t) \\
d \varepsilon=\Delta \sinh (t) d t \\
\frac{\varepsilon^{2}}{\Delta^{2}}-1=\sinh ^{2}(t)
\end{gathered}
$$

ficamos com

$$
\begin{aligned}
I_{n} & =n \Delta^{5} \int_{0}^{\infty} \sinh ^{2}(t) \cosh ^{3}(t) e^{-n \frac{\Delta}{T} \cosh (t)} d t \\
& =n \Delta^{5} \int_{0}^{\infty}\left[\cosh ^{5}(t)-\cosh ^{3}(t)\right] e^{-n \frac{\Delta}{T} \cosh (t)} d t \\
& =n \frac{\Delta^{5}}{16} \int_{0}^{\infty}[\cosh (5 t)+\cosh (3 t)-2 \cosh (t)] e^{-n \frac{\Delta}{T} \cosh (t)} d t \\
& =n \frac{\Delta^{5}}{16}\left[K_{5}\left(n \frac{\Delta}{T}\right)+K_{3}\left(n \frac{\Delta}{T}\right)-2 K_{1}\left(n \frac{\Delta}{T}\right)\right]
\end{aligned}
$$

onde 


$$
K_{m}(x)=\int_{0}^{\infty} \cosh (m t) e^{-x \cosh (t)} d t
$$

são as funções de Bessel modificadas de segunda classe, com $x=n \frac{\Delta}{T}$.

Usando a relação de recorrência das funções de Bessel:

$$
K_{m+1}(x)=K_{m-1}(x)+\frac{2 m}{x} K_{m}(x)
$$

temos

$$
K_{5}(x)+K_{3}(x)-2 K_{1}(x)=\frac{8}{x}\left[K_{2}(x)+K_{4}(x)\right]
$$

Então:

$$
I_{n}=\frac{1}{2} \Delta^{4} T\left[K_{2}\left(n \frac{\Delta}{T}\right)+K_{4}\left(n \frac{\Delta}{T}\right)\right]
$$

Finalmente o calor específico é dado pela seguinte expressão:

$$
c_{m}\left(T, H_{e x t}=0\right)=\frac{R}{4 \pi^{2} c_{1} \sqrt{c_{2}}} \frac{\Delta^{4}}{T} \sum_{n=1}^{\infty}\left[K_{2}\left(n \frac{\Delta}{T}\right)+K_{4}\left(n \frac{\Delta}{T}\right)\right]
$$

\subsubsection{Limite de altas temperaturas $(T \gg \Delta)$}

Vamos considerar o limite em que $T \gg \Delta$. Neste limite as funções de Bessel $^{46}\left[K_{n}\left(\frac{n \Delta}{T}\right)\right]$, são iguais a:

$$
\begin{aligned}
& K_{2}\left(\frac{n \Delta}{T}\right) \approx \frac{1}{2}\left(\frac{2 T}{n \Delta}\right)^{2} \\
& K_{4}\left(\frac{n \Delta}{T}\right) \approx 3\left(\frac{2 T}{n \Delta}\right)^{4}
\end{aligned}
$$

Então o termo dominante é:

$$
c_{m}\left(T, H_{e x t}=0\right)=\frac{R \pi^{2}}{15 c_{1} \sqrt{c_{2}}} T^{3}
$$

onde usamos que

$$
\sum_{n=1}^{\infty} \frac{1}{n^{4}}=\zeta(4)=\frac{\pi^{4}}{90}
$$

\subsubsection{Limite de baixas temperaturas $(T \ll \Delta)$}

Neste limite assintótico as funções de $\operatorname{Besse}^{46}$ são iguais a:

$$
K_{n}(x) \approx \sqrt{\frac{\pi}{2}} \frac{e^{-x}}{x^{1 / 2}}\left[1+0\left(\frac{1}{x}\right)\right]
$$

Então, considerando só o primeiro termo do somatorio $(n=1)$, o calor específico se escreve como:

$$
c_{m}\left(T, H_{e x t}=0\right) \simeq \frac{R}{(2 \pi)^{3 / 2} c_{1} \sqrt{c_{2}}} \frac{\Delta^{7 / 2}}{T^{1 / 2}} e^{-\frac{\Delta}{T}}\left[1+0\left(\frac{T}{\Delta}\right)\right]
$$




\subsection{Caso quase-bidimensional}

Se $H_{A}=0$ e $J_{2} \ll J_{1}$ o sistema é quase bidimensional. Então fazendo uma expansão em potências de $\left(\frac{J_{2}}{J 1}\right)$ na relação de dispersão (3.29) obtemos:

$$
\omega_{k}=\delta+J_{1} S a^{2}\left(k_{x}^{2}+k_{y}^{2}\right)
$$

com um gap pequeno $\delta=8 J_{2} S$.

Usando as equações (4.1) e (4.5) e fazendo a substituição do somatório por uma integral dentro da zona de Brillouin (ZB):

$$
c_{m}=\frac{R}{2 \pi} \frac{1}{T^{2}} \int_{Z B} \frac{\omega_{k}^{2} e^{\frac{\omega_{k}}{T}}}{\left(e^{\frac{\omega_{k}}{T}}-1\right)^{2}} d^{2} k
$$

Substituindo (4.25) em (4.26):

$$
c_{m}=\frac{R}{2 \pi} \frac{1}{T^{2}} \int_{0}^{\infty} \frac{\left(\delta+J_{1} S a^{2} k^{2}\right)^{2}}{\left(e^{\frac{\delta+J_{1} S a^{2} k^{2}}{T}}-1\right)^{2}} k d k
$$

onde estendemos o limite superior da integral a $\infty$, válido no limite de temperaturas baixas.

Fazendo a mudança de variável $x=\frac{J_{1} S}{T} k^{2}$, temos

$$
c_{m}=\frac{R}{2 \pi} \frac{T}{J_{1} S} \int_{0}^{\infty} \frac{\left(\frac{\delta}{T}+x\right)^{2} e^{\frac{\delta}{T}+x}}{\left(e^{\frac{\delta}{T}+x}-1\right)^{2}} d x
$$

Desenvolvendo em series de potências de $\frac{\delta}{T}$ e integrando, obtemos a seguinte expressão para o calor específico:

$$
c_{m}=\frac{\pi R}{12}\left[\frac{1}{J_{1} S} T-0(\delta)\right]
$$

\subsection{Aplicações aos borocarbetos}

Vejamos agora como estas aproximações se aplicam aos diversos borocarbetos. No capítulo 2 vimos que a série em estudo $(\mathrm{R}=\mathrm{Gd}, \mathrm{Ho}, \mathrm{Er}$, Dy, $\mathrm{Tb}$ e $\mathrm{Nd}$ ) apresenta uma ampla variedade de estruturas magnéticas na ausência de campo magnético externo e que estão resumidas na tabela (2.3).

\subsection{1 $G d N i_{2} B_{2} C$}

A temperaturas $T<3.5 K$ este composto apresenta uma estrutura magnética $\mathrm{AF} 3 \mathrm{D}$, sem gap $(\Delta \simeq 0)$ na relação de dispersão dos magnons, 


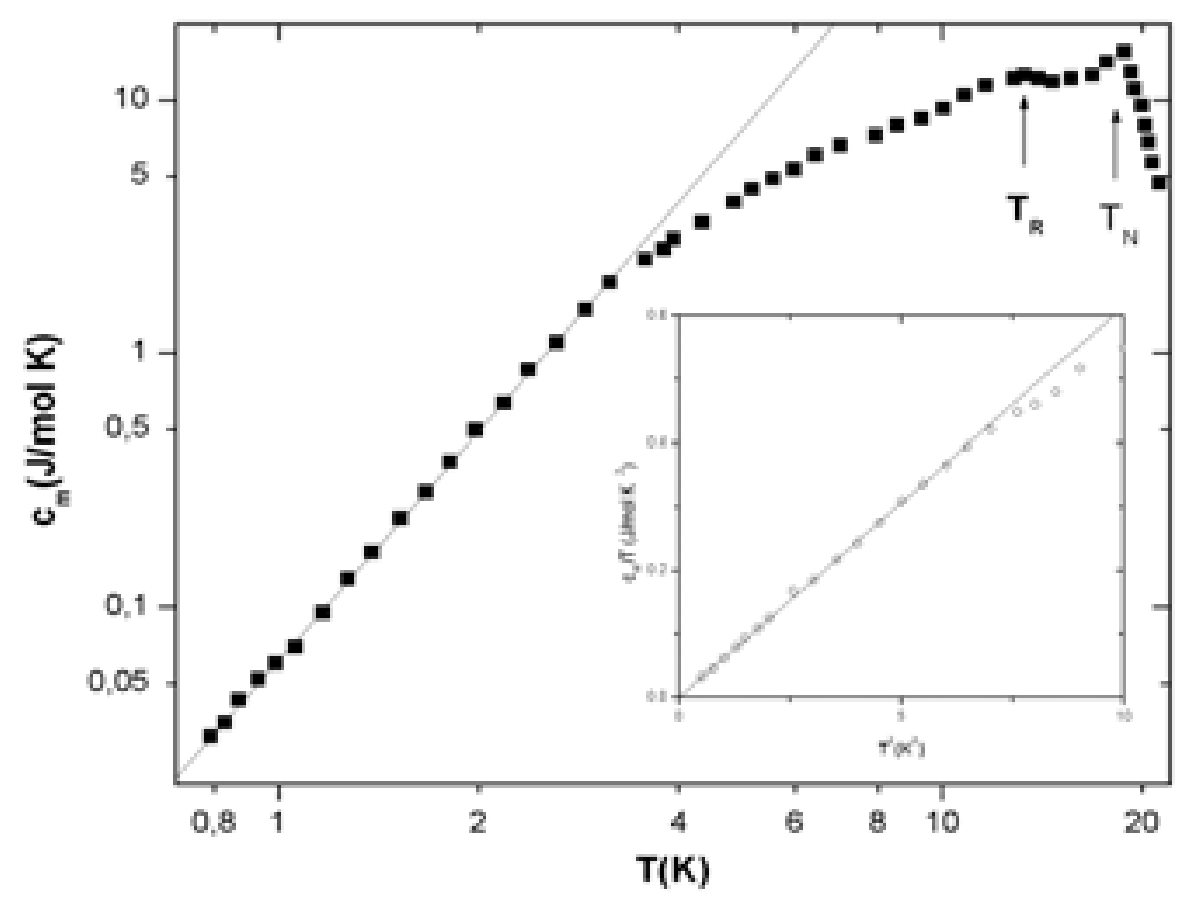

Figura 4.1: Calor específico do $G d N i_{2} B_{2} C$. A linha sólida correspode a resultados teóricos para $c_{1} \sqrt{c_{2}}=173.9 K^{3}$ e os pontos representam resultados experimentais ${ }^{12}$.

a qual implica que $H_{A} \simeq 0$ de acordo com a equação (3.31). Então a contribuição dos magnons ao calor específico no nosso modelo é dada por (4.21). Para $c_{1} \sqrt{c_{2}} \approx 173.9 K^{3}$, o resultado obtido se aproxima bem do resultado experimental (vide figura 4.1 ).

\subsection{2 $\mathrm{HoNi}_{2} \mathrm{~B}_{2} \mathrm{C}$}

Este composto possui uma estrutura AF 3D, mostrada na figura 2.7(a). Os momentos magnéticos são forçados a alinhar-se na direção do eixo fácil $\langle 110\rangle$. O sistema pode ser dividido em duas subredes FM, que interagem AF. O espectro de energia das ondas de spin é anisotropico (com um gap $\Delta \approx 8.5 K)$. A contribuição dos magnons ao calor específico vem dado pela expressão (4.19) e a baixas temperaturas pela expressão (4.24). Para $c_{1} \sqrt{c_{2}} \approx 87.8 K^{3}$, estas expressões descrevem bem os resultados experimentais muma faixa de temperatura que vai até cerca de $4 \mathrm{~K}$ (vide figura 4.2).

\subsection{3 $\mathrm{ErNi}_{2} \mathrm{~B}_{2} \mathrm{C}$}

No capítulo anterior vimos que este composto apresenta duas fases magnéticas entre os intervalos de temperatura: $T_{W F}<T<T_{N}$ e $T<T_{W F}$. Na faixa de temperatura $T<T_{W F}\left(T_{W F}=2.2 K\right)$ a relação de dispersào das ondas de spin é $\mathrm{AF} 3 \mathrm{D}$ com gap, $\Delta \approx 5.4 K$, o calor específico é bem descrito 


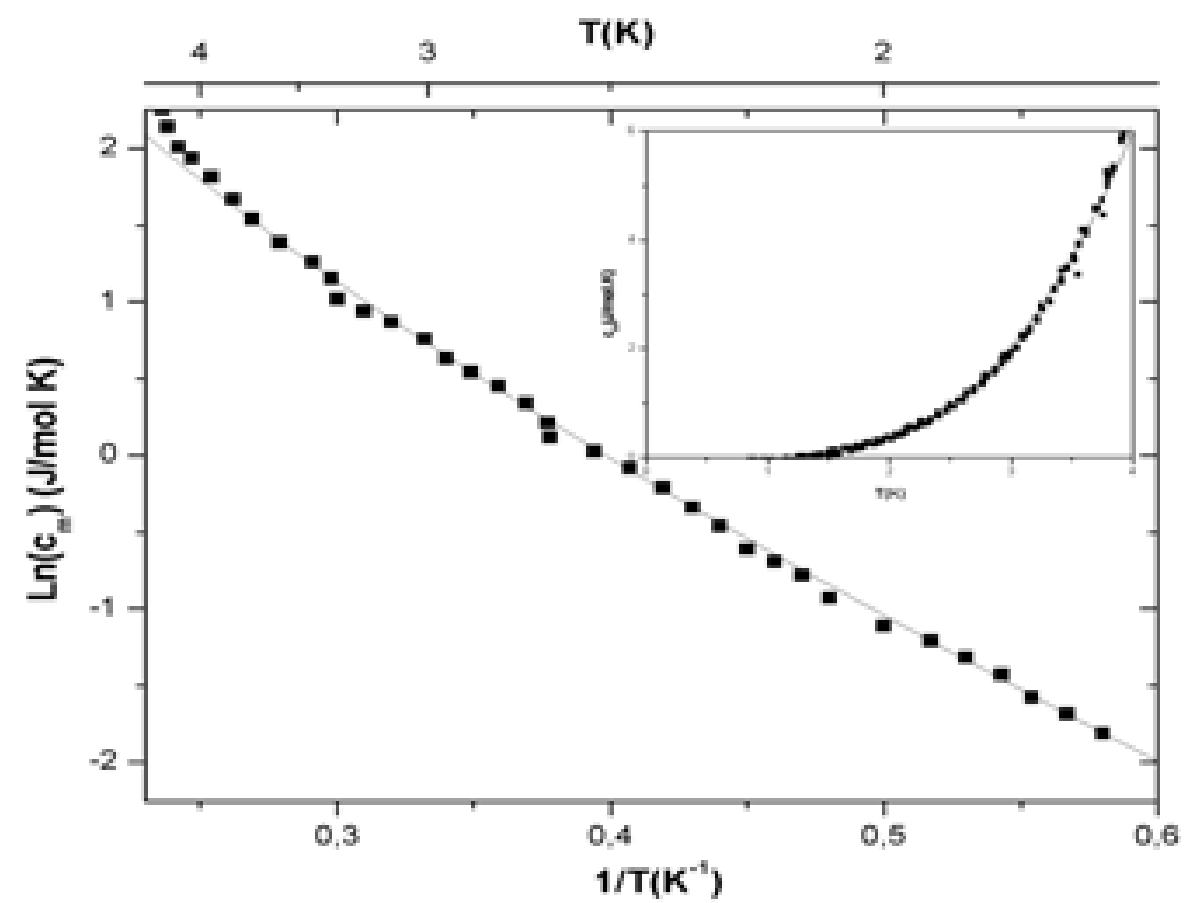

Figura 4.2: Calor específico do $\mathrm{HoN}_{2} \mathrm{~B}_{2} \mathrm{C}$. A linha sólida correspode a resultados teóricos para $c_{1} \sqrt{c_{2}}=87.8 K^{3}$ e os pontos representam resultados experimentais ${ }^{12}$.

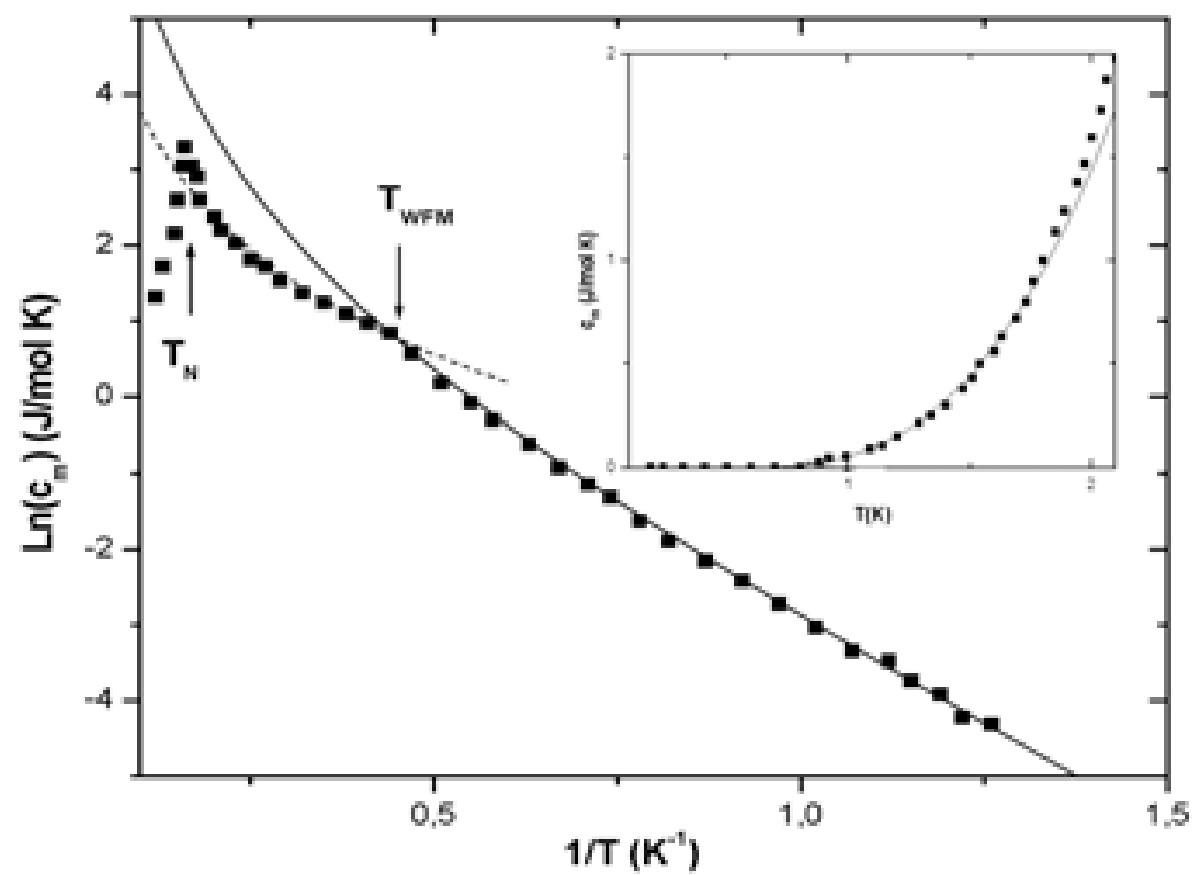

Figura 4.3: Calor específico do $\mathrm{ErNi}_{2} \mathrm{~B}_{2} \mathrm{C}$; a linha sólida correspode a resultados teóricos para $c_{1} \sqrt{c_{2}}=38.9 K^{3}$ e os pontos representam resultados experimentais ${ }^{12}$. 


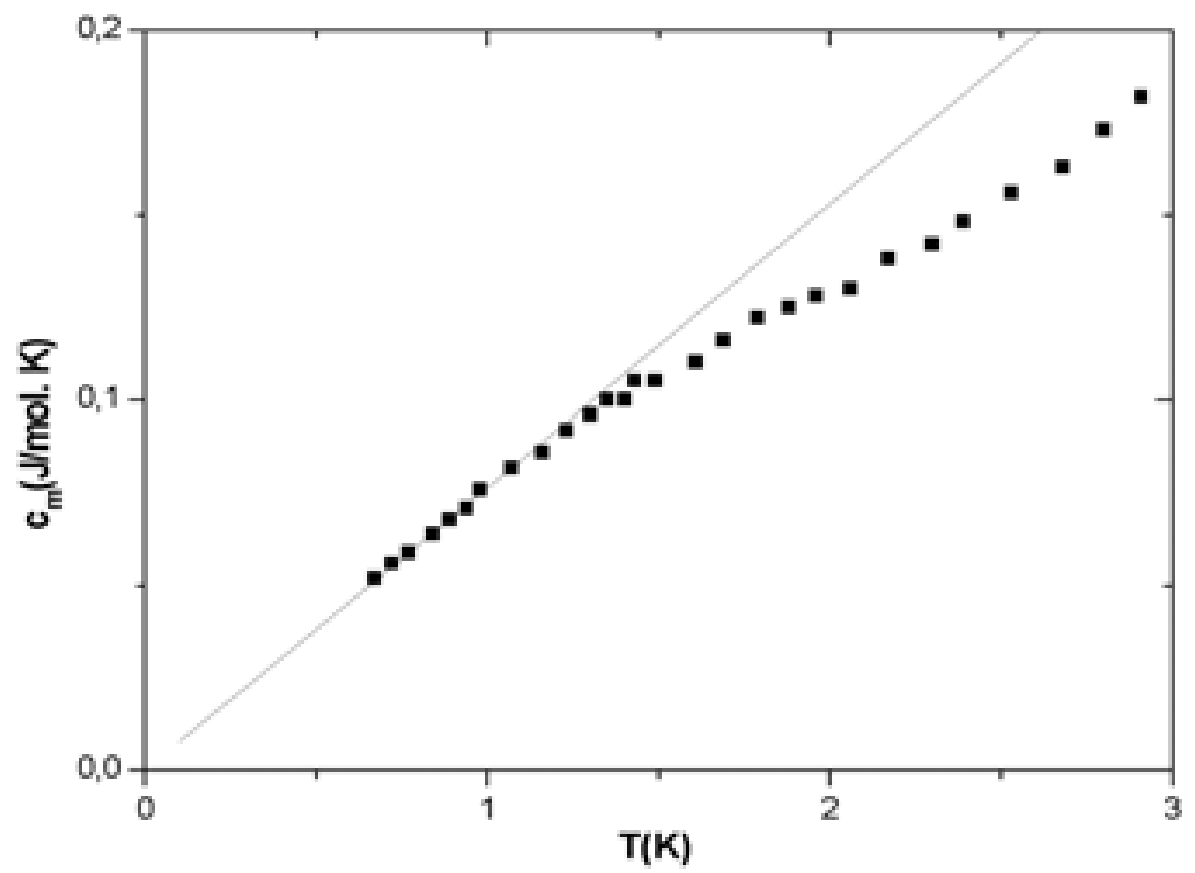

Figura 4.4: Calor específico do $D y N i_{2} B_{2} C$; a linha sólida correspode a resultados teóricos para $J_{1}=3.8 \mathrm{~K}$ e os pontos representam resultados experimentais ${ }^{12}$.

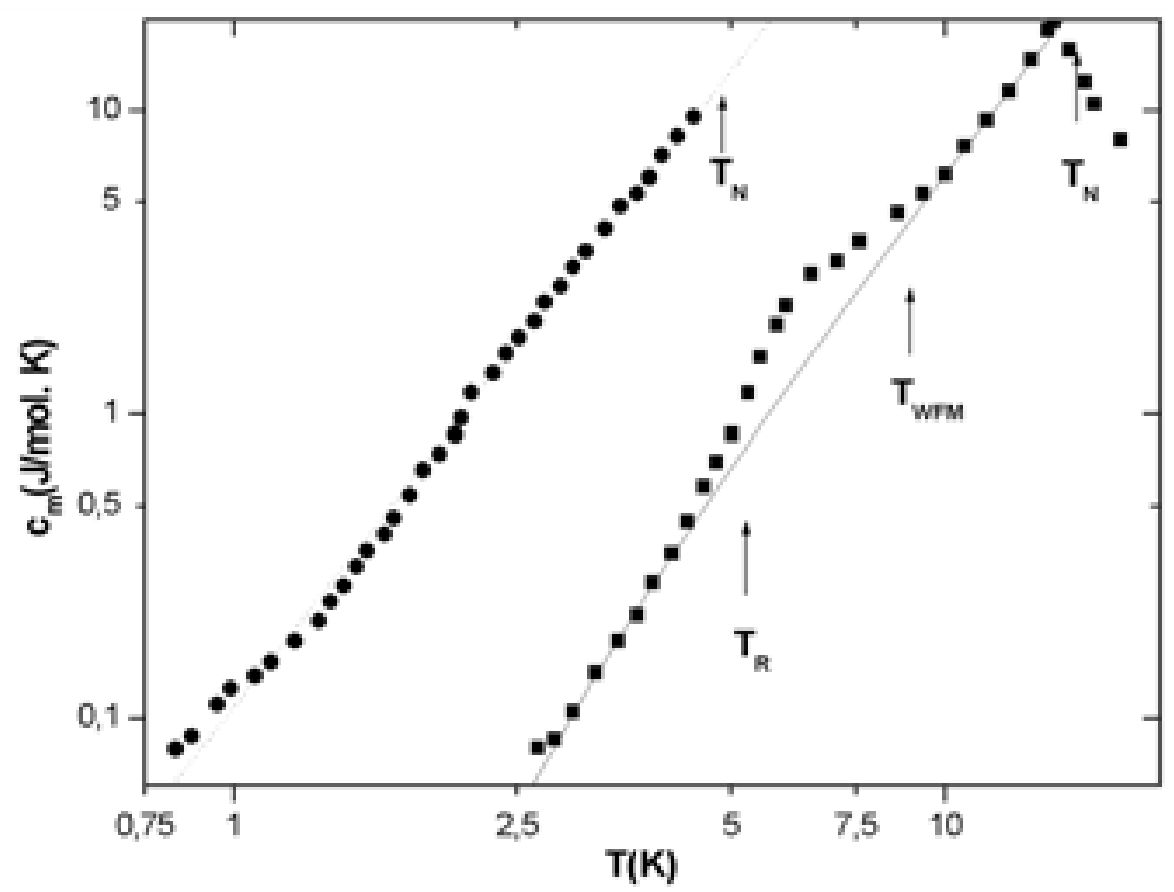

Figura 4.5: Calor específico do $T b N i_{2} B_{2} C$. A linha sólida correspode a resultados teóricos para $c_{1} \sqrt{c_{2}}=1691,6 K^{3}$ e os pontos representam resultados experimentais. A linha pontilhada correspode a resultados teóricos para o $N d N i_{2} B_{2} C\left(c_{1} \sqrt{c_{2}}=50.5 K^{3}\right)$ e os pontos representam resultados experimentais ${ }^{12}$.

pela expressão (4.19) ou (4.24) e com $c_{1} \sqrt{c 2} \approx 38.9 K^{3}$, se aproxima bem ao obtido experimentalmente (vide figura 4.3). 


\subsection{4 $D y N i_{2} B_{2} C$}

Para temperaturas entre $0.5 K<T<2 K$, este composto parece não apresentar gap no espectro de energia dos magnons, a estrutura magnética é FM quase-bidimensional com, $J_{2} \approx 0$. O gap, se existe, é bem pequeno ${ }^{12}$. Então no nosso modelo o calor específico segue a fórmula (4.29) o qual se aproxima bem do obtido experimentalmente para $J_{1} \approx 3.8 \mathrm{~K}$ (vide figura 4.4).

\subsection{5 $\mathrm{TbN}_{2} \mathrm{~B}_{2} \mathrm{C}$}

Este composto abaixo de $T_{R}(=5 K)$ apresenta uma estrutura $\mathrm{AF}^{12} 3 \mathrm{D}$, então o calor específico vem dado por (4.19). Com $c_{1} \sqrt{c_{2}} \approx 1691.6 K^{3} \mathrm{e}$ $\Delta=8.9 \mathrm{~K}$ se aproxima bem ao obtido experimentalmente (vide figura 4.5).

\subsection{6 $N d N i_{2} B_{2} C$}

Este composto apresenta anomalia próximo ${ }^{12}$ de $T=1 K$. Longe desta anomalia a estrutura magnética é $\mathrm{AF} 3 \mathrm{D}$ com gap nulo, na relação de dispersão dos magnons. O calor específico tem o comportamento dado por (4.21) e se aproxima bastante bem aos resultados experimentais para $c_{1} \sqrt{c_{2}} \approx 50.5$ (vide figura 4.5).

Vemos que as curvas do calor específico dos borocarbetos podem ser sistematizadas em função da constante $c_{1} \sqrt{c_{2}}$, para quase todos os compostos, com exceção do Dy. As cosntantes $c_{1}$ e $c_{2}$ são funções de $J_{1}, J_{2}$. Podemos tomar $J_{2}$ como um parâmetro básico. $\mathrm{O}$ campo de anisotropia $H_{A}$ fica determinado por $J_{2}$ (consideramos $\Delta$ como um parâmetro extraido da experiência) e $J_{1}$ é função de $J_{2}$ através de $c_{1} \sqrt{c_{2}}$. Para determinarmos $J_{2}$, entretanto, precisaríamos ajustar mais uma propriedade física.

\begin{tabular}{||c|c|c|c|c|c|c|c|c|c|c||}
\hline \hline$J_{1}(K)$ & 2.01 & 0.99 & 0.65 & 0.47 & 0.36 & 0.28 & 0.22 & 0.17 & 0.14 & 0.10 \\
\hline$J_{2}(K)$ & 0.01 & 0.02 & 0.03 & 0.04 & 0.05 & 0.06 & 0.07 & 0.08 & 0.09 & 0.10 \\
\hline$J_{1} / J_{2}$ & 201.1 & 49.75 & 21.63 & 11.77 & 7.2 & 4,7 & 3.17 & 2.18 & 1.5 & 1.01 \\
\hline \hline
\end{tabular}

Tabela 4.1: $J_{1}$ e $J_{2}$ compatíveis com $c_{1} \sqrt{c_{2}}=87,8 K^{3}$.

Para o composto do Ho, figura 4.6 mostra os valores de $J_{1}$ em função de $J_{2}$ compativeis com $c_{1} \sqrt{c_{2}}=87.8 K^{3}$ (ver também tabela 4.1). Por outro lado, podemos calcular o calor específico $c_{m}\left(T, H_{e x t}=0\right)$ apartir de (4.1), integrando numericamente na ZB, usando a relação de dispersão aproximada (3.36). Neste caso $c_{m}\left(T, H_{e x t}=0\right)$ depende separadamente de $J_{1}$ e $J_{2}$. Na 


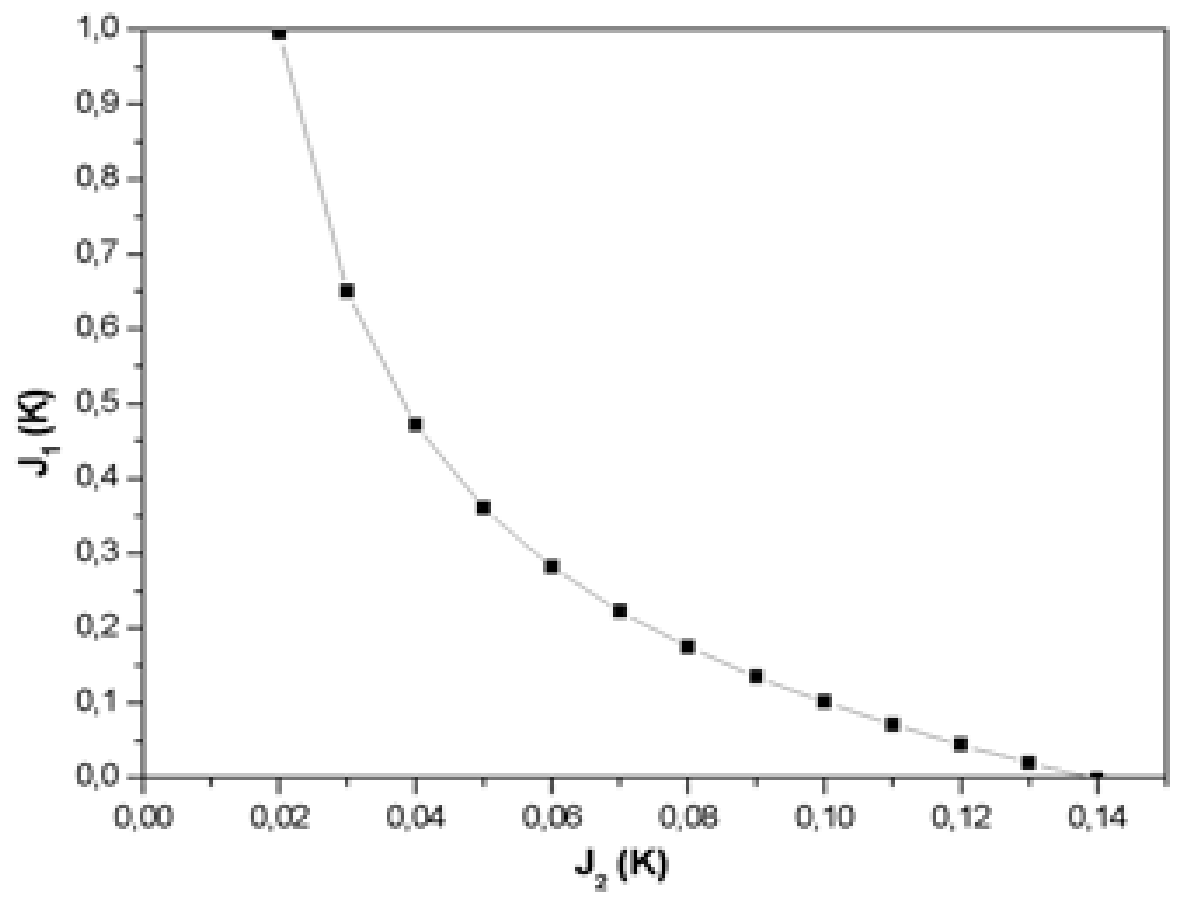

Figura 4.6: $J_{1}$ vs. $J_{2}$ para o $\mathrm{HoNi}_{2} \mathrm{~B}_{2} \mathrm{C}$.

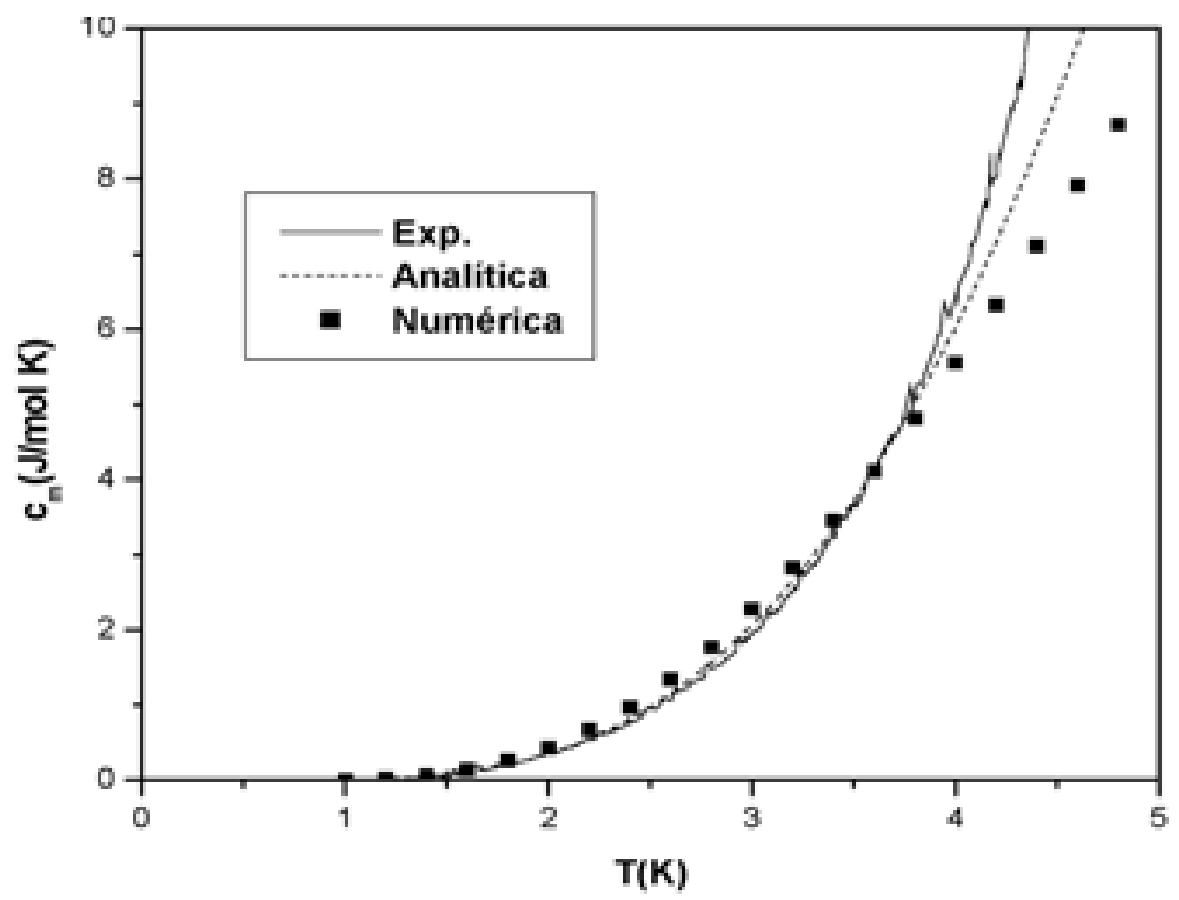

Figura 4.7: Calor específico do $\mathrm{HoNi}_{2} \mathrm{~B}_{2} \mathrm{C}$. A linha sólida representa resultados experimentais e a linha pontilhada e os pontos representam resultados teóricos usando a relação analítica e integrando na ZB respectivamente para $c_{1} \sqrt{c_{2}}=87,8 K^{3}$.

figura 4.7 plotamos o calor específico para $J_{2}=0.04 \mathrm{~K}$ e $J_{1}=0.47 \mathrm{~K}$ que ajusta melhor os dados experimentais. Para este composto então o melhor 
jogo de parâmetros é $c_{1} \sqrt{c_{2}}=87.8 K^{3}, J_{2}=0.04 K, J_{1}=0.47 K, H_{A}=6.3 K$ com $\Delta=8.5 K$.

Ussando o modelo com CEF, Canfield et al $l^{47}$ determinaram com $\mathrm{HoNi}_{2} \mathrm{~B}_{2} \mathrm{C}$ $J_{1}=0.5 \mathrm{~K}$ e $J_{2}=0.06 \mathrm{~K}$, valores que são bastante próximos dos obtidos no presente trabalho. 


\section{Calor específico em função da temperatura e do campo magnético externo}

Neste capítulo abordamos o estudo do comportamento do calor específico em função da temperatura e de um campo magnético externo $\left(H_{e x t}\right)$. A aplicação de um campo magnético nestes compostos dá como resultado o surguimento de transições de fase, chamadas metamagnéticas, que em muitos casos foram mapeados usando medidas de magnetização, calor específico, etc. Com o incremento do campo magnético externo foram observados, no $\mathrm{HoNi}_{2} \mathrm{~B}_{2} \mathrm{C}$ até quatro estados magnéticos estaveis diferentes:

- a) o estado fundamental $(\uparrow \downarrow)$.

- b) o primeiro estado metamagnético $(\uparrow \uparrow \downarrow)$.

- c) o segundo estado metamagnético $(\uparrow \uparrow \rightarrow)$.

- d) o estado paramagnético saturado $(\uparrow)$.

As setas $\uparrow, \downarrow$ e $\rightarrow$ representam os momentos locais, alinhados ao longo do eixo [110] que são respectivamente paralelos, antiparalelos e perpendiculares à direção do campo aplicado. Como se pode ver na figura 5.1, estas fases estão separadas por três transições de fase em $H_{c_{1}}, H_{c_{2}}$ e $H_{c_{3}}$. Neste capítulo calculamos $c_{m}\left(T, H_{e x t}\right)$ para a fase antiferromagnética na qual $H<H_{c_{1}}$ e aplicamos para o caso do $\mathrm{HoN}_{2} \mathrm{~B}_{2} \mathrm{C}$. Nosso modelo só se aplica à fase AF $\left(H_{e x t}<H_{c_{1}}\right)$ e veremos como o campo magnético aplicado contribui ao calor específico.

Nos cálculos que se seguem percorremos os mesmos passos do caso em ausência de campo magnético externo realizados no capítulo anterior.

\subsection{Densidade de estados}

A densidade de estados na presença de um campo magnético externo para cada ramo é

$$
\nu \mathcal{D}^{ \pm}\left(\varepsilon_{ \pm}\right)=\frac{V}{(2 \pi)^{3}} \int \frac{d S_{\varepsilon_{ \pm}}}{\left|\nabla_{\vec{k}} \omega_{\vec{k}}^{ \pm}\right|}
$$

onde $\varepsilon_{ \pm}=\omega_{k}^{ \pm}=\omega_{k} \pm 2 \mu_{0} H_{e x t}$, V é o volume do sistema e $d S_{\varepsilon_{ \pm}}$é o elemento de área no espaço $\mathrm{k}$ da superficie de energia constante e igual a $\varepsilon_{ \pm}$.

Claramente 


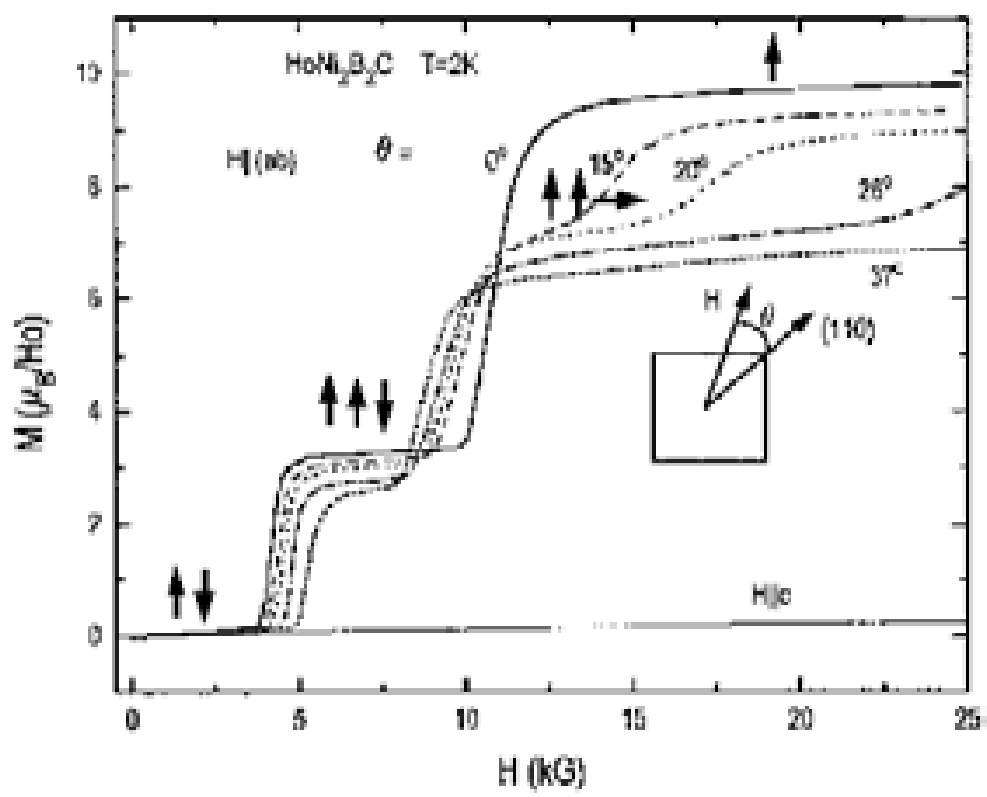

Figura 5.1: Magnetização $\mathrm{M}$ vs campo magnético aplicado $\mathrm{H}$ para o $\mathrm{HoN} i_{2} \mathrm{~B}_{2} \mathrm{C}$ em $T=2 K$ para $\mathrm{H} \| c$ e $\mathrm{H} \perp c$. Para $\mathrm{H} \perp c$ o campo magnético faz um angulo $\theta$ com relação ao eixo cristalográfico $[110]^{48}$.

$$
\mathcal{D}^{ \pm}\left(\varepsilon_{ \pm}\right)=\frac{R}{4 \pi^{2} c_{1} \sqrt{c_{2}}}\left(\varepsilon_{ \pm} \mp 2 \mu_{0} H_{\text {ext }}\right)\left[\left(\varepsilon_{ \pm} \mp 2 \mu_{0} H_{\text {ext }}\right)^{2}-\Delta^{2}\right]^{1 / 2}
$$

pois

$$
\mathcal{D}^{ \pm}\left(\varepsilon_{ \pm}\right)=\mathcal{D}\left(\varepsilon_{ \pm} \mp 2 \mu_{0} H_{\text {ext }}\right)
$$

uma vez que o efeito do campo magnético externo é apenas de deslocar rigidamente a banda correspondente a $H_{\text {ext }}=0$ (ver figura 5.2).

\subsection{Energia Interna}

Em equilíbrio térmico, a energia interna das excitações das ondas de spin, é então:

$$
\begin{aligned}
E= & E_{0}+\nu \int_{\Delta+2 \mu_{0} H_{e x t}}^{\infty} \varepsilon_{+} \mathcal{D}\left(\varepsilon_{+}-2 \mu_{0} H_{e x t}\right)\left\langle n_{\varepsilon_{+}}\right\rangle d \varepsilon_{+} \\
& +\nu \int_{\Delta-2 \mu_{0} H_{e x t}}^{\infty} \varepsilon_{-} \mathcal{D}\left(\varepsilon_{-}+2 \mu_{0} H_{e x t}\right)\left\langle n_{\varepsilon_{-}}\right\rangle d \varepsilon_{-}
\end{aligned}
$$

onde $E_{0}$ é constante independente da temperatura.

Em forma análoga ao caso para $H_{e x t}=0$, as integrais foram estendidas a $\infty$, por que a contribuição para $\varepsilon$ grande é desprezivel devido ao termo exponencial no denominador. 


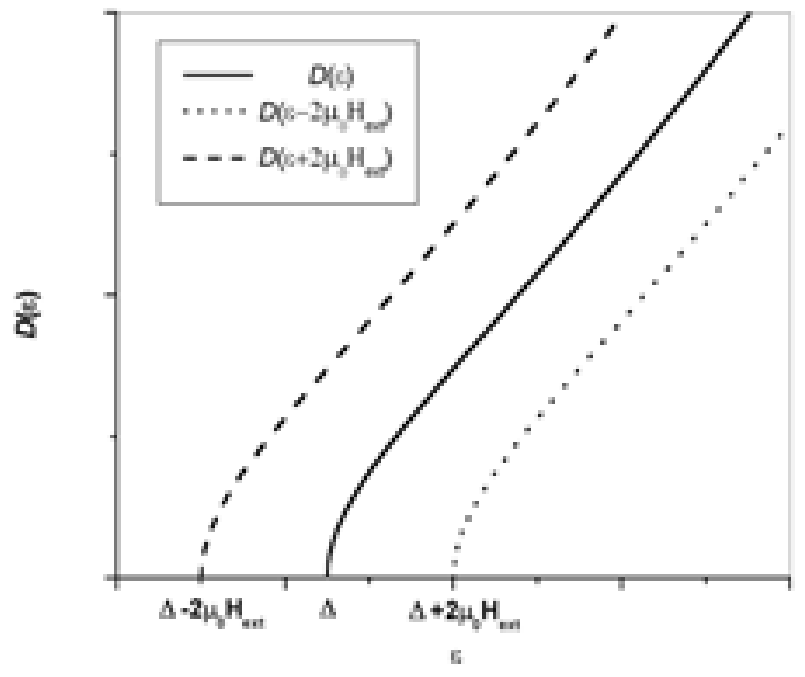

Figura 5.2: Densidade de estados dos magnons com um campo magnético aplicado.

\subsection{Calor específico}

Com as equações (4.5) e (5.4) escrevemos o calor específico em função da temperatura e do campo magnético externo:

$$
\begin{aligned}
c_{m}\left(T, H_{e x t}\right)= & \frac{1}{k_{B} T^{2}}\left\{\int_{\Delta+2 \mu_{0} H_{e x t}}^{\infty} \frac{\varepsilon_{+}^{2} \mathcal{D}\left(\varepsilon_{+}-2 \mu_{0} H_{e x t}\right) e^{\beta \varepsilon_{+}}}{\left(e^{\beta \varepsilon_{+}}-1\right)^{2}} d \varepsilon_{+}\right. \\
& \left.+\int_{\Delta-2 \mu_{0} H_{e x t}}^{\infty} \frac{\varepsilon_{-}^{2} \mathcal{D}\left(\varepsilon_{+}+2 \mu_{0} H_{e x t}\right) e^{\beta \varepsilon_{-}}}{\left(e^{\beta \varepsilon_{-}}-1\right)^{2}} d \varepsilon_{-}\right\}
\end{aligned}
$$

Fazendo a mesma aproximação realizada no capítulo anterior, expressamos o calor específico como:

$$
c_{m}\left(T, H_{e x t}\right)=\frac{\phi}{T^{2}} \sum_{n=1}^{\infty} n\left[I_{n}+J_{n}\right]
$$

com

$$
\begin{aligned}
& \phi I_{n}=\int_{\Delta+2 \mu_{0} H_{e x t}}^{\infty} \varepsilon_{+}^{2} \mathcal{D}\left(\varepsilon_{+}-2 \mu_{0} H_{e x t}\right) e^{-n \beta \varepsilon_{+}} d \varepsilon_{+} \\
& \phi J_{n}=\int_{\Delta-2 \mu_{0} H_{e x t}}^{\infty} \varepsilon_{-}^{2} \mathcal{D}\left(\varepsilon_{+}+2 \mu_{0} H_{e x t}\right) e^{-n \beta \varepsilon_{-}} d \varepsilon_{-}
\end{aligned}
$$

onde $\phi=\frac{R}{4 \pi^{2} c_{1} \sqrt{c_{2}}}$.

Substituindo a densidade de estados, equação (5.2), obtemos:

$I_{n}=\int_{\Delta+2 \mu_{0} H_{e x t}}^{\infty} \varepsilon_{+}^{2}\left(\varepsilon_{+}-2 \mu_{0} H_{e x t}\right)\left[\left(\varepsilon_{+}-2 \mu_{0} H_{e x t}\right)^{2}-\Delta^{2}\right]^{1 / 2} e^{-n \beta \varepsilon_{+}} d \varepsilon_{+}$ 
Fazendo a mudança de variavel $u=\varepsilon_{+}-2 \mu_{0} H_{\text {ext }}$ :

$$
\begin{aligned}
I_{n}=e^{-n \beta\left(2 \mu_{0} H_{e x t}\right)} & \left\{\int_{\Delta}^{\infty} u^{3}\left(u^{2}-\Delta^{2}\right)^{1 / 2} e^{-n \beta u} d u\right. \\
+ & 2\left(2 \mu_{0} H_{e x t}\right) \int_{\Delta}^{\infty} u^{2}\left(u^{2}-\Delta^{2}\right)^{1 / 2} e^{-n \beta u} d u \\
+ & \left.\left(2 \mu_{0} H_{e x t}\right)^{2} \int_{\Delta}^{\infty} u\left(u^{2}-\Delta^{2}\right)^{1 / 2} e^{-n \beta u} d u\right\}
\end{aligned}
$$

Fazendo uma nova mudança de variavel $u=\Delta \cosh (t)$, em forma análoga ao realizado no capítulo anterior, encontramos

$$
\begin{aligned}
I_{n}=e^{-n \beta\left(2 \mu_{0} H_{e x t}\right)} & \left\{\frac{\Delta^{5}}{16} \int_{0}^{\infty}[\cosh (5 t)+\cosh (3 t)-2 \cosh (t)] e^{-n \frac{\Delta}{T} \cosh (t)} d t\right. \\
+ & \frac{\Delta^{4}}{4}\left(2 \mu_{0} H_{e x t}\right) \int_{0}^{\infty}(\cosh (4 t)-1) e^{-n \frac{\Delta}{T} \cosh (t)} d t \\
+ & \left.\frac{\Delta^{3}}{4}\left(2 \mu_{0} H_{e x t}\right)^{2} \int_{0}^{\infty}(\cosh (3 t)-\cosh (t)) e^{-n \frac{\Delta}{T} \cosh (t)} d t\right\} \\
I_{n}=e^{-n \beta\left(2 \mu_{0} H_{e x t}\right)} & \left\{\frac{\Delta^{5}}{16}\left[K_{5}\left(n \frac{\Delta}{T}\right)+K_{3}\left(n \frac{\Delta}{T}\right)-2 K_{1}\left(n \frac{\Delta}{T}\right)\right]\right. \\
+ & \frac{\Delta^{4}}{4}\left(2 \mu_{0} H_{e x t}\right)\left[K_{4}\left(n \frac{\Delta}{T}\right)-K_{0}\left(n \frac{\Delta}{T}\right)\right] \\
+ & \left.\frac{\Delta^{3}}{4}\left(2 \mu_{0} H_{e x t}\right)^{2}\left[K_{3}\left(n \frac{\Delta}{T}\right)-2 K_{1}\left(n \frac{\Delta}{T}\right)\right]\right\}
\end{aligned}
$$

onde $K_{n}\left(\frac{n \Delta}{T}\right)$ são as funções de Bessel modificadas de segunda classe, definidas na equação (4.15). Usando a relação de recorrência (4.16) obtemos

$$
\begin{aligned}
I_{n} & =e^{-n \beta\left(2 \mu_{0} H_{e x t}\right)}\left\{\frac{\Delta^{4} T}{2 n}\left[K_{2}\left(n \frac{\Delta}{T}\right)+K_{4}\left(n \frac{\Delta}{T}\right)\right]\right. \\
& \left.+\frac{\Delta^{4}}{4}\left(2 \mu_{0} H_{e x t}\right)\left[K_{4}\left(n \frac{\Delta}{T}\right)-K_{0}\left(n \frac{\Delta}{T}\right)\right]+\frac{\Delta^{2} T}{n}\left(2 \mu_{0} H_{e x t}\right)^{2}\left[K_{2}\left(n \frac{\Delta}{T}\right)\right]\right\}
\end{aligned}
$$

Analogamente

$$
\begin{aligned}
J_{n} & =e^{n \beta\left(2 \mu_{0} H_{e x t}\right)}\left\{\frac{\Delta^{4} T}{2 n}\left[K_{2}\left(n \frac{\Delta}{T}\right)+K_{4}\left(n \frac{\Delta}{T}\right)\right]\right. \\
& \left.-\frac{\Delta^{4}}{4}\left(2 \mu_{0} H_{e x t}\right)\left[K_{4}\left(n \frac{\Delta}{T}\right)-K_{0}\left(n \frac{\Delta}{T}\right)\right]+\frac{\Delta^{2} T}{n}\left(2 \mu_{0} H_{e x t}\right)^{2}\left[K_{2}\left(n \frac{\Delta}{T}\right)\right]\right\}
\end{aligned}
$$

Finalmente obtemos a seguinte expressão para o calor específico:

$$
\begin{aligned}
c_{m}\left(T, H_{e x t}\right)= & \frac{R}{4 \pi^{2} c_{1} \sqrt{c_{2}}} \frac{\Delta^{4}}{T^{2}} \sum_{n=1}^{\infty}\left\{T\left[K_{2}\left(n \frac{\Delta}{T}\right)+K_{4}\left(n \frac{\Delta}{T}\right)\right] \cosh \left[n \frac{2 \mu_{0} H_{e x t}}{T}\right]\right. \\
& -\frac{n}{2}\left(2 \mu_{0} H_{e x t}\right)\left[K_{4}\left(n \frac{\Delta}{T}\right)-K_{0}\left(n \frac{\Delta}{T}\right)\right] \sinh \left[n \frac{2 \mu_{0} H_{e x t}}{T}\right] \\
& \left.+2\left(\frac{2 \mu_{0} H_{e x t}}{\Delta}\right)^{2} T K_{2}\left(n \frac{\Delta}{T}\right) \cosh \left[n \frac{2 \mu_{0} H_{e x t}}{T}\right]\right\}
\end{aligned}
$$




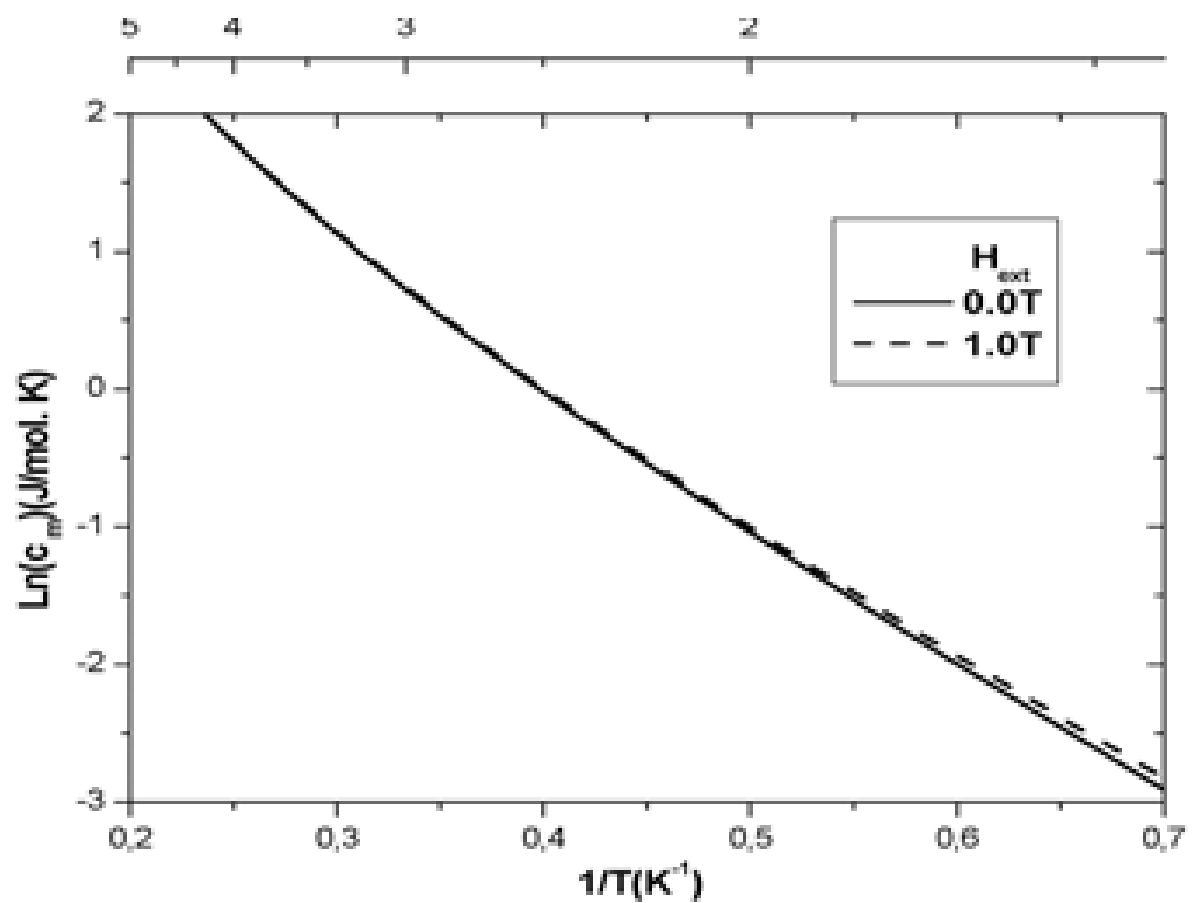

Figura 5.3: Calor específico do $\mathrm{HoNi}_{2} \mathrm{~B}_{2} \mathrm{C}$ em função do campo magnético aplicado para $T=2 K$, para $c_{1} \sqrt{c_{2}}=87.8 K^{3}$.

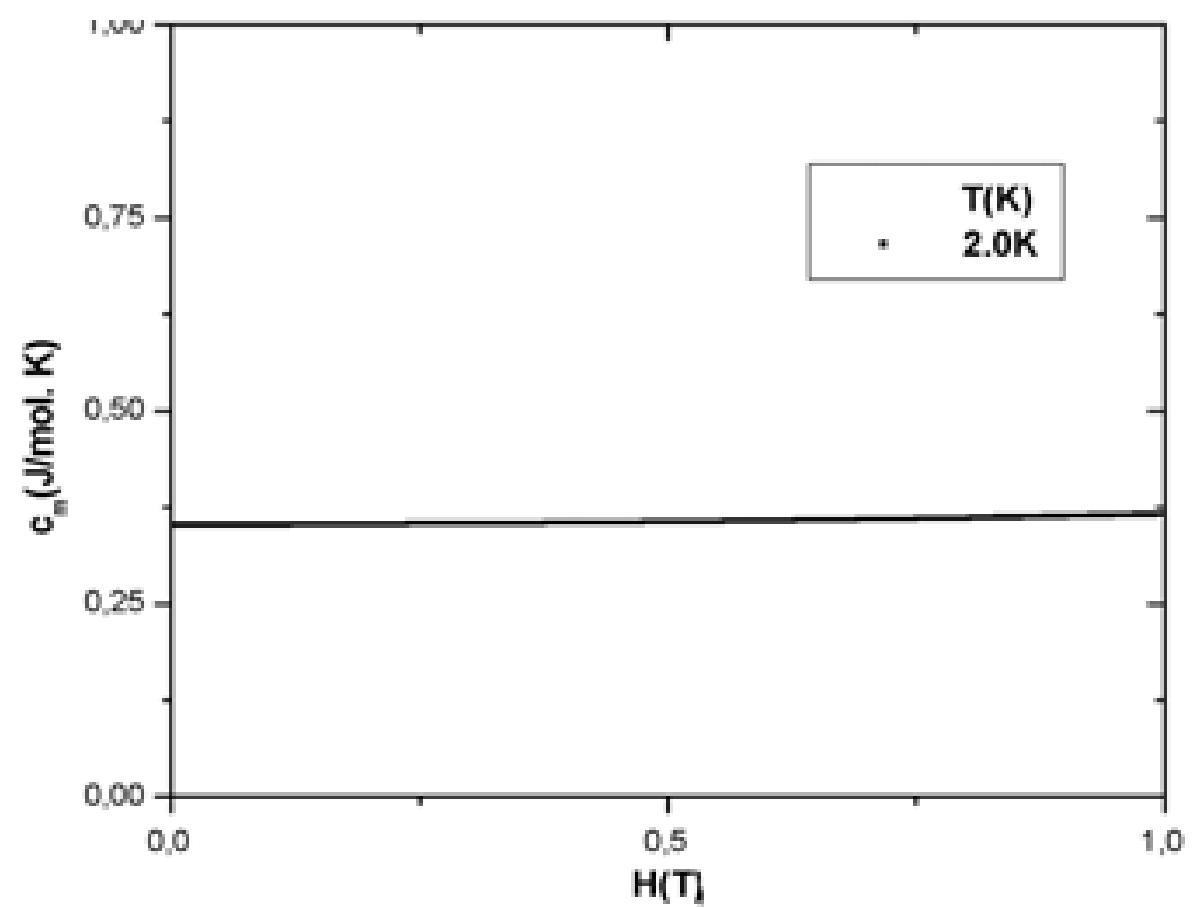

Figura 5.4: Calor específico do $\mathrm{HoNi}_{2} \mathrm{~B}_{2} \mathrm{C}$ em função do campo magnético aplicado para $T=2 K$, para $c_{1} \sqrt{c_{2}}=87.8 K^{3}$. 
Vemos que se o campo externo é nulo, o calor específico se reduz à equação (4.19).

Vejamos agora como este resultado se aplica ao $\mathrm{HoNi}_{2} \mathrm{~B}_{2} \mathrm{C}$. Da figura 5.4 vemos que na fase $\operatorname{AF}\left(H<H_{c_{1}}\right)$ o calor específico depende fracamente do campo magnético aplicado o qual indica que a densidade de magnons não sofre uma mudança apreciável. Usamos $c_{1} \sqrt{c_{2}}=87.8 K^{3}$ obtido do ajuste do calor específico na ausência de campo magnético. Nossos resultados mostram a pouca dependencia do calor específico do campo magnético nesta fase. Isto está de acordo com o resultado experimental mostrado na figura 5.1, pois a magnetização - e portanto o número de magnons - depende muito pouco do campo magnético. 


\section{6 \\ Magnetização}

Neste capítulo calculamos a magnetização de subrede na fase AF usando a teoria de ondas de spin. Na primeira parte fazemos o cálculo do efeito das flutuações quânticas, integrando na zona de Brillouin e na segunda parte apresentamos o cálculo da magnetização em função da temperatura e do campo magnético. Aplicamos o modelo para o composto $\mathrm{HoNi}_{2} \mathrm{~B}_{2} \mathrm{C}$.

\subsection{Magnetização de subrede}

Nesta seção calculamos a magnetição da subrede A. O spin total na direção z é dado por:

$$
S_{A}^{z}=\sum_{j \in A} S_{A, j}^{z}
$$

Substituindo a expressão de $S_{A, j}^{z}$ (equação (3.2)) em (6.1):

$$
\begin{gathered}
S_{A}^{z}=N S-\frac{1}{N} \sum_{j, \vec{k}, \overrightarrow{k^{\prime}}} e^{i\left(\vec{k}-\overrightarrow{k^{\prime}}\right) \cdot \vec{x}_{j}} c_{\vec{k}}^{+} c_{\overrightarrow{k^{\prime}}} \\
=N S-\sum_{\vec{k}, \overrightarrow{k^{\prime}}} \underbrace{\left(\frac{1}{N} \sum_{j} e^{i\left(\vec{k}-\overrightarrow{k^{\prime}}\right) \cdot \vec{x}_{j}}\right)}_{\delta_{\vec{k}, \overrightarrow{k^{\prime}}}} c_{\vec{k}}^{+} c_{\overrightarrow{k^{\prime}}}
\end{gathered}
$$

onde N é o número de sitos na subrede. Somando em $\overrightarrow{k^{\prime}}$ :

$$
S_{A}^{z}=N S-\sum_{\vec{k}} c_{\vec{k}}^{+} c_{\vec{k}}
$$

Com a transformação canônica, (3.20) e (3.21), a equação anterior se escreve como:

$$
S_{A}^{z}=N S-\sum_{\vec{k}}\left(u_{\vec{k}}^{2} \alpha_{\vec{k}}^{+} \alpha_{\vec{k}}+v_{\vec{k}}^{2} \beta_{\vec{k}}^{+} \beta_{\vec{k}}+v_{\vec{k}}^{2}+\cdots\right)
$$

\subsection{Magnetização da subrede a $T=0 K$}

Em primeiro lugar calculamos a magnetização a $T=0 K$, quando $n_{\vec{k}}^{ \pm}=0$. Então tomando a média térmica de $S_{A}^{z}$ obtemos: 


$$
\left\langle S_{A}^{z}(0)\right\rangle=N S-\sum_{\vec{k}} v_{\vec{k}}^{2}
$$

Das equações 3.23 e 3.24 :

$$
\left\langle S_{A}^{z}(0)\right\rangle=N S-\frac{1}{2} \sum_{\vec{k}}\left(\frac{A_{\vec{k}}}{\omega_{\vec{k}}}-1\right)
$$

onde o primeiro termo nos dá a magnetização de saturação e o segundo termo representa o efeito da oscilação de ponto zero, que reduz a magnetização de saturação devido às flutuações quânticas. Então

$$
\Delta S_{A}^{z}(0) \equiv N S-\left\langle S_{A}^{z}(0)\right\rangle=\frac{1}{2} \sum_{\vec{k}}\left(\frac{A_{\vec{k}}}{\omega_{\vec{k}}}-1\right)
$$

Vemos também que em $T=0 K$, como era de se esperar, a magnetização é independente do campo externo.

Fazendo a mudança do somatório sobre $\vec{k}$ para integral, obtemos

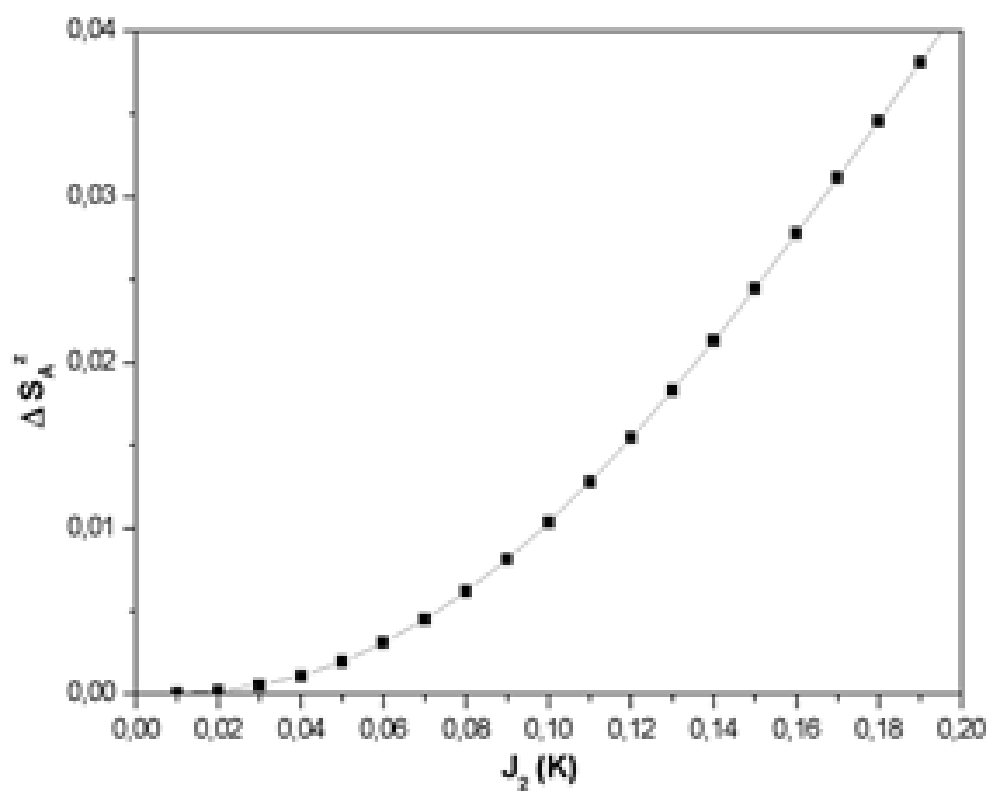

Figura 6.1: Variação de $\Delta S_{A}^{z}(T=0 K)$ vs. $J_{2}$ para o $H o N i_{2} B_{2} C$.

$$
\Delta S_{A}^{z}(0)=\frac{N a^{2} c}{2(2 \pi)^{3}} \int_{Z . B .}\left[\frac{A_{\vec{k}}}{\omega_{\vec{k}}}-1\right] d^{3} \vec{k}
$$

onde $\frac{a^{2} c}{2}$ é o volume da célula cristalina.

Usando a relação (6.8), calculamos a flutuação quântica por sitio para o composto $\mathrm{HoNi}_{2} \mathrm{~B}_{2} \mathrm{C}$. Na figura 6.1 mostramos $\Delta S_{A}^{z}(0)$ para vários valores de $J_{2}$ (ver também a tabela 6.1). Como explicado anteriormente para o Ho, os parâmetros apropriados são $J_{2}=0.04 K$ e $J_{1}=0.47 K$ o que dá $\Delta S_{A}^{z}(0)=0.002$ 


\begin{tabular}{||c|c|c|c|c|c|c|c|c|c|c||}
\hline \hline$J_{2}(K)$ & 0.01 & 0.02 & 0.03 & 0.04 & 0.05 & 0.06 & 0.07 & 0.08 & 0.09 & 0.10 \\
\hline$\Delta S_{A}^{z} / \mathrm{N}$ & 0.00017 & 0.00052 & 0.001 & 0.002 & 0.003 & 0.004 & 0.006 & 0.008 & 0.010 & 0.012 \\
\hline \hline
\end{tabular}

Tabela 6.1: $J_{1}$ e $J_{2}$ compatíveis com $c_{1} \sqrt{c_{2}}=87.8 K^{3}$.

\subsection{Magnetização da subrede a $T \neq 0 K$}

Agora calculamos a magnetização a $T \neq 0 K$ e com um campo magnético externo aplicado $\left(H_{\text {ext }}\right)$. Neste caso, as médias térmicas do número de ocupação dos magnons $\left(n_{\vec{k}}\right)$ são diferente de zero. Das equações (3.30) e (6.4):

$$
\left\langle S_{A}^{z}\left(T, H_{e x t}\right)\right\rangle=\left\langle S_{A}^{z}(0)\right\rangle-\sum_{\vec{k}}\left(u_{\vec{k}}^{2}\left\langle n_{\vec{k}}^{+}\right\rangle+v_{\vec{k}}^{2}\left\langle n_{\vec{k}}^{-}\right\rangle\right)
$$

Substituindo $u_{\vec{k}}^{2}$ e $v_{\vec{k}}^{2}$ vem

$$
\begin{aligned}
\left\langle S_{A}^{z}\left(T, H_{e x t}\right)\right\rangle & =\left\langle S_{A}^{z}(0)\right\rangle-\frac{1}{2} \sum_{\vec{k}}\left[\left(\frac{A_{\vec{k}}}{\omega_{\vec{k}}}+1\right)\left\langle n_{\vec{k}}^{+}\right\rangle+\left(\frac{A_{\vec{k}}}{\omega_{\vec{k}}}-1\right)\left\langle n_{\vec{k}}^{-}\right\rangle\right](6.10) \\
\left\langle S_{A}^{z}\left(T, H_{e x t}\right)\right\rangle & =\left\langle S_{A}^{z}(0)\right\rangle-\frac{1}{2} \sum_{\vec{k}} \frac{A_{\vec{k}}}{\omega_{\vec{k}}}\left[\left\langle n_{\vec{k}}^{+}\right\rangle+\left\langle n_{\vec{k}}^{-}\right\rangle\right]-\frac{1}{2} \sum_{\vec{k}}\left[\left\langle n_{\vec{k}}^{+}\right\rangle-\left\langle n_{\vec{k}}^{-}\right\rangle\right]
\end{aligned}
$$

onde $n_{\vec{k}}^{ \pm}$vem dado pela equação (4.3).

O primeiro termo foi calculado na seção anterior; agora calculamos os dois ultimos termos. Antes de calcular estes termos, precisamos encontrar os fatores do segundo e terceiro termos. Com (4.3) achamos:

$$
\begin{aligned}
\left\langle n_{k}^{+}\right\rangle+\left\langle n_{k}^{-}\right\rangle & =\frac{1}{e^{\beta \omega_{k}^{+}}-1}+\frac{1}{e^{\beta \omega_{k}^{-}}-1} \\
& =\frac{e^{-\beta \omega_{k}^{+}}}{1-e^{-\beta \omega_{k}^{+}}}+\frac{e^{-\beta \omega_{k}^{-}}}{1-e^{-\beta \omega_{k}^{-}}}
\end{aligned}
$$

Usando a relação:

$$
\frac{1}{1-x}=\sum_{n=0}^{\infty} x^{n}
$$

a relação (6.12) fica:

$$
\begin{aligned}
\left\langle n_{k}^{+}\right\rangle+\left\langle n_{k}^{-}\right\rangle & =\sum_{n=1}^{\infty}\left(e^{-n \beta \omega_{k}^{+}}+e^{-n \beta \omega_{k}^{-}}\right) \\
& =2 \sum_{n=1}^{\infty} e^{-n \frac{\omega_{k}}{T}} \cosh \left[n \frac{2 \mu_{0} H_{e x t}}{T}\right]
\end{aligned}
$$


Analogamente para o terceiro termo de (6.11):

$$
\left\langle n_{k}^{+}\right\rangle-\left\langle n_{k}^{-}\right\rangle=-2 \sum_{n=1}^{\infty} e^{-n \frac{\omega_{k}}{T}} \sinh \left[n \frac{2 \mu_{0} H_{e x t}}{T}\right]
$$

Substituindo na equação (6.11) as equações (6.14) e (6.15) :

$$
\begin{aligned}
\left\langle S_{A}^{z}\left(T, H_{e x t}\right)\right\rangle= & \left\langle S_{A}^{z}(0)\right\rangle-\sum_{n=1}^{\infty}\left\{\left[\sum_{\vec{k}} \frac{A_{\vec{k}}}{\omega_{\vec{k}}} e^{-n \frac{\omega_{k}}{T}}\right] \cosh \left[n \frac{2 \mu_{0} H_{e x t}}{T}\right]\right. \\
& \left.-\left[\sum_{\vec{k}} e^{-n \frac{\omega_{k}}{T}}\right] \sinh \left[n \frac{2 \mu_{0} H_{e x t}}{T}\right]\right\}
\end{aligned}
$$

Fazendo a mudança do somatório sobre $\vec{k}$ para integral e as mesmas aproximações feitas no cálculo do calor específico, no regime de baixas temperaturas quando $\frac{\hbar \omega_{\vec{k}}^{ \pm}}{k_{B} T}>1$ e a integral pode ser extendida sobre todo o espaço, obtemos

$$
\begin{aligned}
\sum_{\vec{k}} \frac{A_{\vec{k}}}{\omega_{\vec{k}}} e^{-n \beta \omega_{k}}= & \frac{V}{(2 \pi)^{3}} \int_{Z . B .} \frac{A_{\vec{k}}}{\omega_{\vec{k}}} n_{\vec{k}} d^{3} \vec{k} \\
= & \frac{N \Delta^{2}}{(2 \pi)^{2} c_{1} \sqrt{c_{2}}}\left\{\left(8 J_{2} S+2 \mu_{0} H_{A}\right)\left[K_{2}\left(\frac{n \Delta}{T}\right)-K_{0}\left(\frac{n \Delta}{T}\right)\right]\right. \\
& \left.+\frac{J_{1} S \Delta^{2}}{6 c_{1}}\left[K_{4}\left(\frac{n \Delta}{T}\right)-4 K_{2}\left(\frac{n \Delta}{T}\right)+3 K_{0}\left(\frac{n \Delta}{T}\right)\right]\right\}
\end{aligned}
$$

onde $K_{n}\left(\frac{n \Delta}{T}\right)$ são as funções de Bessel modificadas de segunda classe, definidas na equação (4.15). Usando a relação de recorrência (4.16) obtemos

$$
\begin{aligned}
\sum_{\vec{k}} \frac{A_{\vec{k}}}{\omega_{\vec{k}}} e^{-n \beta \omega_{k}}= & \frac{N \Delta^{2}}{(2 \pi)^{2} c_{1} \sqrt{c_{2}}}\left\{2\left(8 J_{2} S+2 \mu_{0} H_{A}\right) \frac{T}{n \Delta} K_{1}\left(\frac{n \Delta}{T}\right)\right. \\
& \left.+\frac{4 J_{1} S}{n^{2} c_{1}} K_{2}\left(\frac{n \Delta}{T}\right)\right\}
\end{aligned}
$$

e analogamente:

$$
\sum_{\vec{k}} e^{-n \beta \omega_{k}}=\frac{N \Delta^{2}}{2 \pi^{2} c_{1} \sqrt{c_{2}}} \frac{T}{n} K_{2}\left(\frac{n \Delta}{T}\right)
$$

Finalmente:

$$
\begin{aligned}
\frac{\left\langle S_{A}^{z}\left(T, H_{\text {ext }}\right)>\right.}{N} & =\frac{\left\langle S_{A}^{z}(0)\right\rangle}{N} \\
& -\frac{T \Delta}{2 \pi^{2} c_{1} \sqrt{c_{2}}} \sum_{n=1}^{\infty}\left\{\left[\frac{1}{n}\left(8 J_{2} S+2 \mu_{0} H_{A}\right) K_{1}\left(\frac{n \Delta}{T}\right)\right.\right. \\
& \left.+2 \frac{J_{1} S}{c_{1}} \frac{T \Delta}{n^{2}} K_{2}\left(\frac{n \Delta}{T}\right)\right] \cosh \left[\frac{n 2 \mu_{0} H_{e x t}}{T}\right] \\
& \left.-\frac{\Delta}{n} K_{2}\left(\frac{n \Delta}{T}\right) \sinh \left[\frac{n 2 \mu_{0} H_{e x t}}{T}\right]\right\}
\end{aligned}
$$




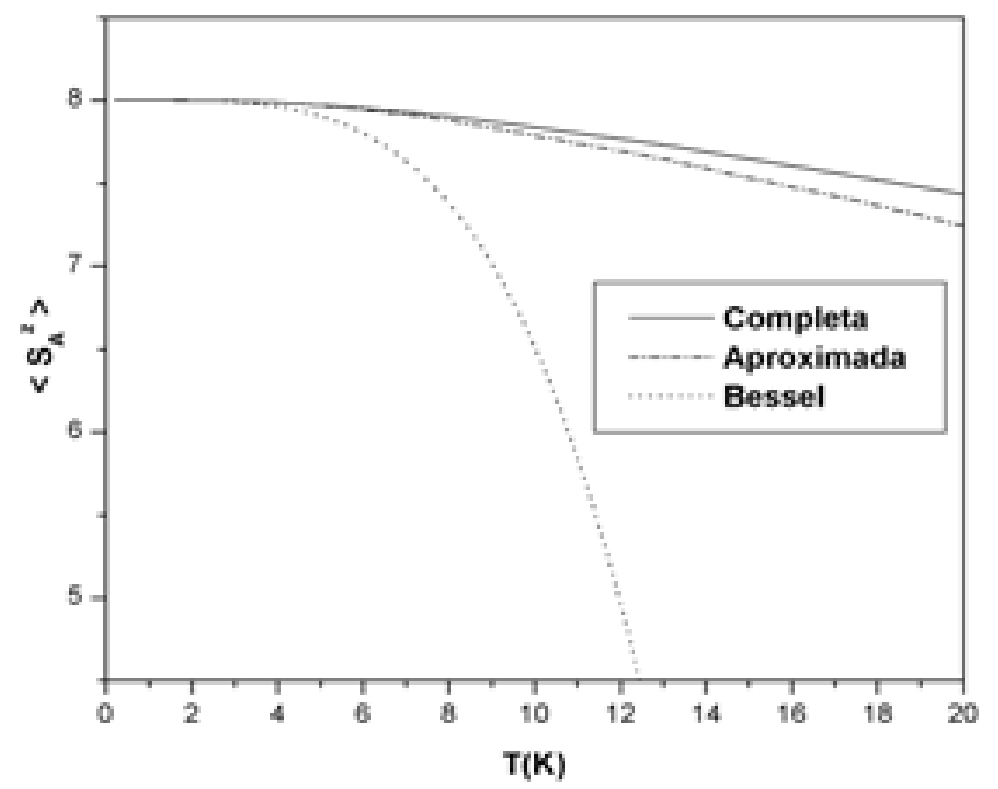

Figura 6.2: Magnetização da subrede $S_{A}^{z}$ vs. T para o $\mathrm{HoNi} i_{2} B_{2} \mathrm{C}$ na ausência de campo magnético externo.

Se o $\overrightarrow{H_{e x t}}=0$, então a magnetização é:

$$
\begin{aligned}
\frac{\left\langle S_{A}^{z}(T, 0)>\right.}{N} & =\frac{\left\langle S_{A}^{z}(0)\right\rangle}{N}-\frac{T \Delta}{2 \pi^{2} c_{1} \sqrt{c_{2}}} \sum_{n=1}^{\infty}\left[\frac{1}{n}\left(8 J_{2} S+2 \mu_{0} H_{A}\right) K_{1}\left(\frac{n \Delta}{T}\right)\right. \\
& \left.+2 \frac{J_{1} S}{c_{1}} \frac{T \Delta}{n^{2}} K_{2}\left(\frac{n \Delta}{T}\right)\right]
\end{aligned}
$$

Para o composto $\mathrm{HoNi}_{2} \mathrm{~B}_{2} \mathrm{C}$, mostramos na figura 6.2 a magnetização da subrede em função da temperatura integrando numéricamente dentro da zona de Brillouin usando tanto a relação de dispersão completa (3.29) como também a aproximada (3.36) e a relação (6.20) encontrada analiticamente. Nesta figura também se observa que na região de aplicabilidade de nosso modelo $(T \leq 5 K)$ as três expressões dão resultados similares.

Na figura 6.3 mostramos o comportamento da magnetização da subrede em função da temperatura para um campo magnético externo de 0.0T, $1.0 \mathrm{~T}$ e $4.0 \mathrm{~T}$ usando a relação (6.20). Observa-se que até aproximadamente $4 \mathrm{~K}$ o campo magnético não produz mudança apreciável na magnetização da subrede. Na figura 6.4 mostramos a magnetização da subrede em função do campo magnético aplicado para $T=2 K$, onde se observa que até $2 \mathrm{~T}$ usando relação (6.20)a magnetização varia muito pouco.

\subsubsection{Limite de baixas temperaturas $T \ll \Delta$}

Neste limite assintótico as funções de Bessel $^{46}$ obedecem a: 


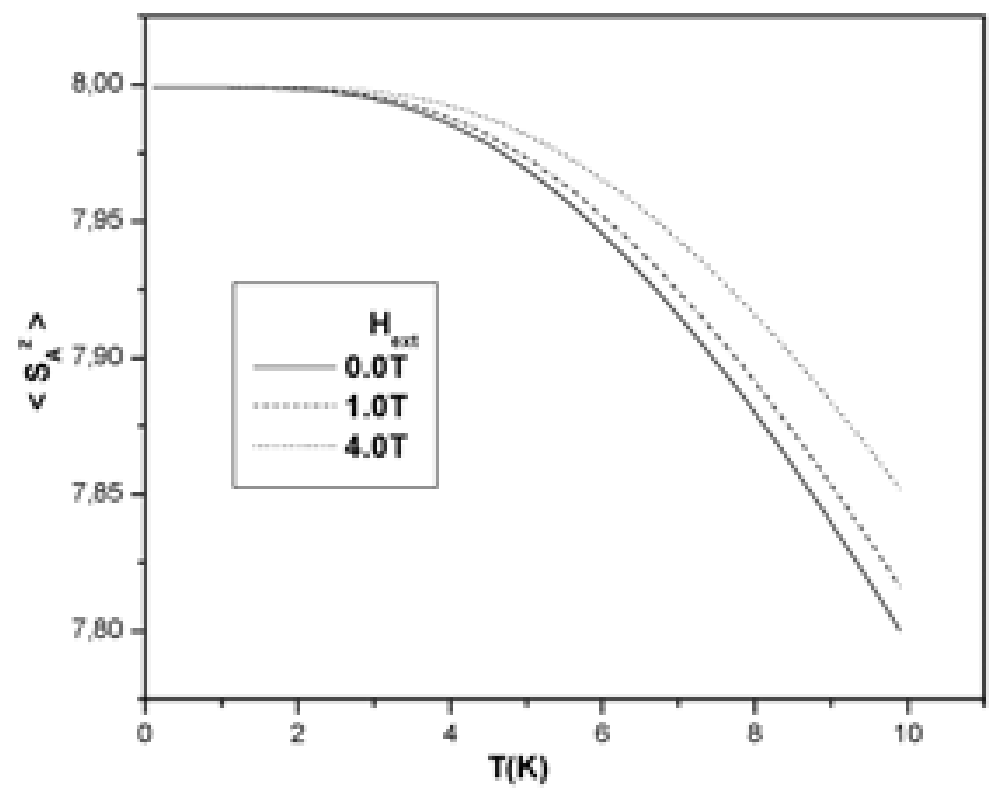

Figura 6.3: Magnetização da subrede $S_{A}^{z}$ vs. $\mathrm{T}$ para um campo magnético aplicado de $H_{e x t}=0.0 \mathrm{~T}, 1.0 \mathrm{~T}$ e $4.0 \mathrm{~T}$ para o $\mathrm{HoN} i_{2} \mathrm{~B}_{2} \mathrm{C}$.

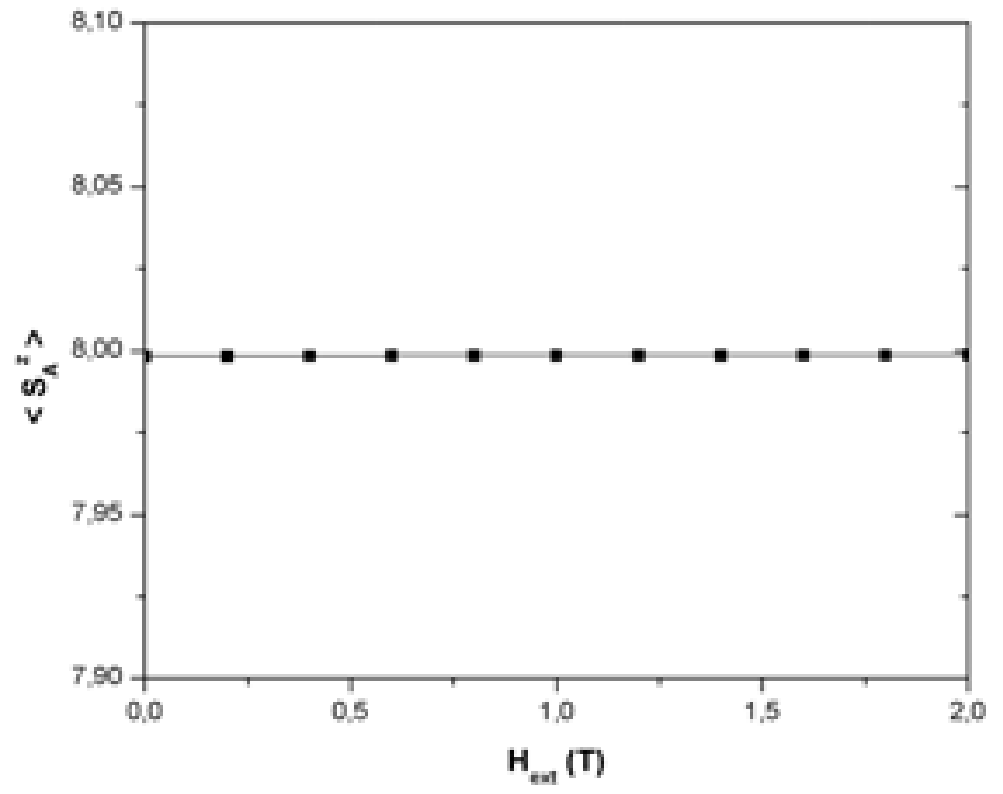

Figura 6.4: Magnetização da subrede $S_{A}^{z}$ vs. campo magnético aplicado a $T=2 \mathrm{~K}$ para o $\mathrm{HoNi}_{2} \mathrm{~B}_{2} \mathrm{C}$. 


$$
K_{n}(x) \approx \sqrt{\frac{\pi}{2}} \frac{e^{-x}}{x^{1 / 2}}\left[1+0\left(\frac{1}{x}\right)\right]
$$

Então, considerando só o primeiro termo do somatorio $(n=1)$, a magnetização na ausência de campo escreve-se como:

$$
\frac{<S_{A}^{z}(T, 0)>}{N} \cong \frac{\left\langle S_{A}^{z}(0)\right\rangle}{N}-\frac{\Delta^{1 / 2}}{(2 \pi)^{3 / 2} c_{1} \sqrt{c_{2}}}\left(8 J_{2} S+2 \mu_{0} H_{A}\right) T^{3 / 2} e^{-\frac{\Delta}{T}}
$$

Desta relação (lei de Bloch) se observa a presença de um fator exponencial que vem da existência de um gap na relação de dispersão dos magnons. Numericamente nosso modelo prevê então

$$
\frac{<S_{A}^{z}(T, 0)>}{N} \simeq 7,998-0.016 T^{3 / 2} e^{\frac{\Delta}{T}}
$$

Ainda não existem dados na literatura para a verificação deste resultado.

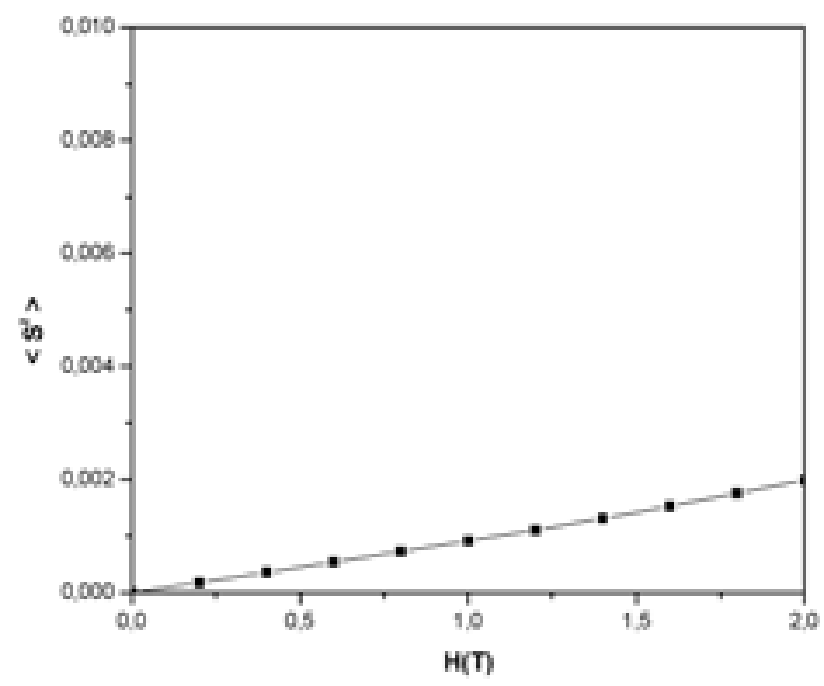

Figura 6.5: Magnetização total $<S^{z}>$ vs. $H_{\text {ext }}$ (em Tesla) a T $=2 \mathrm{~K}$ para o $\mathrm{HoNi}_{2} \mathrm{~B}_{2} \mathrm{C}$.

\subsection{Magnetização total}

Análogo ao cálculo da magnetização da subrede A, calculamos a magnetização da subrede B.

$$
S_{B}^{z}=\sum_{j \in B} S_{B, j}^{z}=-S N+\sum_{\vec{k}} d_{\vec{k}}^{+} d_{\vec{k}}
$$

então 


$$
\left\langle S_{B}^{z}\left(T, H_{e x t}\right)\right\rangle=\left\langle S_{B}^{z}(0)\right\rangle+\sum_{\vec{k}}\left(v_{\vec{k}}^{2}\left\langle n_{\vec{k}}^{+}\right\rangle+u_{\vec{k}}^{2}\left\langle n_{\vec{k}}^{-}\right\rangle\right)
$$

Note a inversão $u_{\vec{k}}, v_{\vec{k}}$ em relação a (6.9).

Substituindo $u_{\vec{k}}$ e $v_{\vec{k}}$ na equação anterior temos

$$
\left\langle S_{B}^{z}\left(T, H_{e x t}\right)\right\rangle=\left\langle S_{B}^{z}(0)\right\rangle+\frac{1}{2} \sum_{\vec{k}}\left[\left(\frac{A_{\vec{k}}}{\omega_{\vec{k}}}-1\right)\left\langle n_{\vec{k}}^{+}\right\rangle+\left(\frac{A_{\vec{k}}}{\omega_{\vec{k}}}+1\right)\left\langle n_{\vec{k}}^{-}\right\rangle\right]
$$

onde

$$
\left\langle S_{B}^{z}(0)\right\rangle=-\left\langle S_{A}^{z}(0)\right\rangle
$$

Somando as relações (6.10) e (6.28) encontamos a magnetização total, que é diferente de zero se $H_{e x t} \neq 0$ :

$$
\left\langle S^{z}(T)\right\rangle=-\sum_{\vec{k}}\left[\left\langle n_{\vec{k}}^{+}\right\rangle-\left\langle n_{\vec{k}}^{-}\right\rangle\right]
$$

$\operatorname{com}(6.15)$ e $(6.19)$

$$
\frac{\left\langle S^{z}(T)\right\rangle}{N}=\frac{\Delta^{2} T}{\pi^{2} c_{1} \sqrt{c_{2}}} \sum_{n} \frac{1}{n} K_{2}\left(\frac{n \Delta}{T}\right) \sinh \left[n \frac{2 \mu H_{e x t}}{T}\right]
$$

Na figura 6.5 mostra-se o comportamento da magnetização total em função do campo magnético aplicado para uma temperatura $T=2 K$. A fraca dependência de $\left\langle S^{z}(T)\right\rangle$ do campo é compatível com o resultado experimental de Canfield et al .

Não há na literatura dados experimentais de $\left\langle S_{A}^{z}\left(T, H_{e x t}\right)\right\rangle$. Nosso modelo permite encontrar $\left\langle S_{A}^{z}(0)\right\rangle,\left\langle S_{A}^{z}(T, 0)\right\rangle$ e $\left\langle S_{A}^{z}\left(T, H_{e x t}\right)\right\rangle$. A comparação com os dados experimentais serviria como teste para o modelo e para as aproximações feitas.

Note que com o ajuste feito com os dados do calor específico, não temos mais nenhum parâmetro ajustável. 


\section{7 \\ Conclusão}

Com o objetivo de estudar as propriedades térmicas e magnéticas dos borocarbetos na fase antiferromagnética, propusemos um hamiltoniano de spin onde o efeito do campo elétrico cristalino foi aproximado por um campo magnético efetivo ou de anisotropia. Usando a teoria de onda de spin encontramos a relação de dispersão e a densidade de estados dos magnons. Com este modelo calculamos o calor específico molar e conseguimos um bom ajuste dos dados experimentais para série $(R=\mathrm{Gd}$, Ho,, Er, Dy, $\mathrm{Tb}, \mathrm{Nd}$ ), obtendo valores físicamente razoáveis para as interações de troca ${ }^{47}$ $J_{1}$ e $J_{2}$, bem como para o campo de anisotropia. O efeito de um campo magnético aplicado sobre a densidade de estados dos magnons é apenas de deslocar rigidamente as bandas. Verificamos que na fase em estudo o campo magnético tem pouca influência no calor específico. Resultados experimentais corroboram este resultado pois revelam que a magnetização depende muito pouco do campo, o que indica que o número de magnons produzidos pela aplicação do campo é muito pequeno.

Calculamos também a magnetização de subrede a $T=0 K$ e a influêcia das flutuações quânticas para o composto $\mathrm{HoNi}_{2} \mathrm{~B}_{2} \mathrm{C}$ e obtivemos $\Delta S_{A}^{z}=0.002$. Como era de se esperar este pequeno valor indica a pouca importância das flutuações quânticas em $d=3$. Calculamos também a dependencia da magnetização de subrede com a temperatura para $T \rightarrow 0$ (lei de Bloch). O resultado revela o efeito dominante do gap o qual produz um decaimento exponencial.

O modelo é válido a baixas temperaturas; para valores mais altos da temperatura o efeito da interação magnon-magnon torna-se importante. Os valores calculados da temperatura de Néel $T_{N}$ sem levar em conta esta interação são muito altos.

Pretendemos levar em conta o efeito desta interação como continuação do

presente trabalho. Também é importante considerar a interação com a rede para explicar quantitativamente as transições presentes no rico diagrama de fase destes compostos. 


\section{Referências}

[1] T. R. J. Cava, H. Takagi, B. Batlogg, H. W. Zendbergen, J. J. Krajewski, W. F. Peck, R. B. Van Dover, R. J. Felder, T. Siegrist, K. Mizvhashi, J. O. Lee, H. Eisaki, S. A. Carter, S. Ushida, Nature 367, 146 (1994)

[2] T. R. J. Cava, H. Takagi, H. W. Zendbergen, J. J. Krajewski, W. J. Peck, T. Siegrist, B. Batlogg, R. B. Van Dover, R. J. Felder, K. Mizvhashi, J. O. Lee, H. Eisaki, S. Ushida Nature 367, 252 (1994)

[3] T. T. Siegrist, H. W. Zendbergen, R. J. Cava, J. J. Krajewski, W. J. Peck, Nature 367, 254 (1994)

[4] R. Nagaraya, Chandan Mazumdar, Zakir Hossain, S. K. Dhar, K. V. Gopalakrishnan, L. C. Gopta, C. Godart, P. D. Padaka and R. Vijayaraghavam, Phys. Rev. Lett. 72, 274 (1994)

[5] J. W. Lynn, S. Skanthakumar, Q. Huang, S. K. Shinha, Z. Hossain, L. C. Gupta, R. Nagarajan and C. Gordat, Phys. Rev. B 55, 6584 (1997)

[6] P. C. Canfield, L. P. Gammel and D. J. Bishop, Physics Today 51, 40 (1998)

[7] M. El Massalami and E. M. Baggio-Saitovitch, J. Magn. and Mag. Materials 153, 97 (1996)

[8] H. Eisaki, H. Takagi, R. J. Cava, K. Mizuhashi, J. O. Lee, B. Batlogg, J. J. Krajewski, W. F. Peck and S. Ushida, Phys. Rev. B 50, 647 (1994)

[9] P. C. Canfield, B. K. Cho and K. W. Dennis, Physica B 215, 337 (1995)

[10] C. Detlefs, A. I. Goldman, C. Stassis, P. C. Canfield, B. K. Cho, J. P. Hill and D. Gibbs Phy. Rev. B 53, 6355 (1996)

[11] M. L. Kulic, A. I. Buzdin and L. N. Bulaevskii, Phy. Letter A 235, 285 (1997)

[12] M. El Massalami, R. E. Rapp e F. A. B. Chaves, comunicação privada.

[13] T. Siegrist, R. J. Cava, J. J. Krajewski and W. F. Peck Jr., J. Alloys Compd 262, 135 (1994)

[14] L. F. Matheiss, Phys. Rev. B 49, 13279 (1994)

[15] W. E. Pickett and D. J. Singh, Phys. Rev. Lett. 72, 3702 (1994) 
[16] L. C. Gupta, R. Nagarajan, Z. Hossain, Z. Mazumdar, S. K. Dhar, C. Godart, C. Levy-Clement, B. D. Padilia and R. Vijayaraghavan, J. Magn. Magn. Mater 140-144, 2053 (1995)

[17] M. E. Massalami, S. L. Bud'ko, B. Giordenengo and E. M. BaggioSaittovitch, Physica C 224, 41 (1995)

[18] R. J. Cava, 'Rare Earth Transition Metal Borocarbides (Nitrides): Superconducting, Magnetic and Normal State Properties', ed. K-H Muller and V. N. Narozhnyi, pg. 21.

[19] K. Winzer and K. Krug, 'Rare Earth Transition Metal Borocarbides (Nitrides): Superconducting, Magnetic and Normal State Properties', ed. K-H Muller and V. N. Narozhnyi, pg. 63.

[20] R. J. Cava, H. W. Zendbergen, B. Batlogg, H. Eisaki, H. Takagi, J. J. Krajewski, W. F. Peck Jr., E. M. Gyorgy and S. Uchida, Nature 372, $245(1994)$

[21] H. W. Zendbergen, J. Jansen, R. J. Cava, J. J. Krajewski and W. F. Peck Jr., Nature 372, 759 (1994)

[22] S. Manalo, H. Michor, M. El-Hagary, G. Hilscher and E. Schachinger, Phys. Rev. B 63, 104508 (2001)

[23] H. Rosner, S-L Drechsler, K. Koepernik, I. Opahle and H. Eschrig, 'Rare Earth Transition Metal Borocarbides (Nitrides): Superconducting, Magnetic and Normal State Properties', ed. K-H Muller and V. N. Narozhnyi, pg. 71.

[24] J. Bardeen, L. N. Cooper and J. R. Schrieffer, Phys. Rev. 106, 162 (1957a)

[25] S-L Drechsler, et al, Physica C 317-8, 117 (1999)

[26] D. J. Singh, Phys. Rev. B 50, 6486 (1994)

[27] D. D. Lawrie and J. P. Franck, Physica C 245, 159 (1995)

[28] V. Metlushco, U. Welp, A. Koshelev, I. Aranson, G. W. Crabtree and P.C. Canfield, Phys. Rev. Lett. 79, 1738 (1997)

[29] H. Tagaky, M. Nohara and R. J. Cava, Physica B 237, 292 (1997)

[30] S. V. Shulga, S-L Drechsler, G. Fuchs, K-H Muller, K. Winzer, M. Heinecke and K. Krug, Phys. Rev. Lett. 80, 1730 (1998) 
[31] S-L Drechsler, H. Rosner, S. Shulga, I. Opahle and H. Eschrig, 'Rare Earth Transition Metal Borocarbides (Nitrides): Superconducting, Magnetic and Normal State Properties', ed. K-H Muller and V. N. Narozhnyi, pg. 403.

[32] T. Tereshima, et al Phys. Rev. B 56, 7843(97)

[33] S. Skanthakumar and J. W. Lynn, Phy. Rev. B 253, 576 (1999)

[34] C. Detlefs, A. I. Goldman, C. Stassis, P. C. Canfield and B. K. Cho, Phys. Rev. B 53, 6355 (1996).

[35] U. Gasser, P. Allenspach, F. Fauth, W. Henggeler, J. Mesot, A. Furrer, S. Rosenkranz, P. Vorderwisch and M. Buchgeister, Z. Phys. B 101, 345-352 (1996).

[36] R. Nagarajan et al, Physica B 259-61, 588(1999)

[37] F. M. Mulder, et al, P. Alloys and Compoun 217, 118(1995)

[38] K. Tomala, et al, Phys. Rev. B 58, 8534(1998)

[39] C. Detlefs, A. H. m. Z. Islam, A. I. Goldman, C. Stassis, P. C. Canfield, J. P. Hill and D. Gibbs, Phys. Rev. B 55, R680 (1997).

[40] B. K. Cho, Physica C, 298, 305 (1998)

[41] I. R. Fisher, J. R. Cooper and P. C. Canfield, Phys. Rev. B 56, 10820 (1997).

[42] R. E. Rapp, F. A. Chaves, M. El Massalami, C. M. Chaves and J. Flores, Physica C 354, 178 (2001)

[43] M. El Massalami, R. E. Rapp, E. F. Chagas, Hiroyuki Takeya, J. Flores and C. M. Chaves J. Phys. Soc. Japan 71, 1 (2002)

[44] S-L Drechsler, H. Rosner, S. Shulga, I. Opahle and H. Eschrig, 'Rare Earth Transition Metal Borocarbides (Nitrides): Superconducting, Magnetic and Normal State Properties', ed. K-H Muller and V. N. Narozhnyi, pg. 223.

[45] C. Kittel, Quantum Theory of Solids (John Wiley and Sons, Inc 1963)

[46] L. Rade e B. Wetergren Mathematics Handbook, fouth Edition, Springer Werlag, 1999

[47] B. K. Cho, B. N. Harmon, D. C. Johnston and P. C. Canfield Phys. Rev. B 53, 2217 (1996). 
[48] P. C. Canfield, S. L. Bud'ko, B. K. Cho, A. Lacerda, D. Farrell, E. Johnston-Halperin, V. A. Kalatsky and V. L. Pokrovsky Phys. Rev. B 55, 970 (1997). 